Time Optimal Slewing of Flexille Spacecraft

b $y^{\prime}$

Joseph Z. Ben-Asher

Dissertation submitted to the Faculty of the

Virginia Polytechnic Institute and State University

in partial fulfillment of the requirements for the degree of

Doctor of Philosophy

in

Aerospace and Ocean Enginecring

APPROVED:

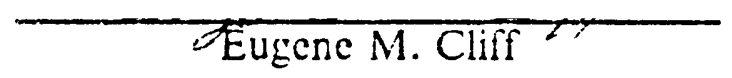

John A. Burns

Frederick XI. Luxze

Raphacl P. Haitka

Stanoje Bingulac

Terry L. Herdman

June, 1988

Blacksourg, Virginia 


\title{
Time Optimal Slewing of Flexible Spacecraft
}

\author{
by \\ Joseph Z. Ben-Asher. \\ Eugene M. Cliff \\ Acrospace and Occan Engineering
}

\section{(ABSTRACT)}

The time optimal slewing problem for flexible spacecraft is considered. We study single-axis rotational maneuvers for a simple flexible system, consisting of a rigid hub with an elastic appendage. The equations of motions are derived by Hamilton's Principle, and a discrete nonlinear model is obtained by the assumed modes method. The problem is first solved in a discrete linearized space by parameter optimization. Optimality is verified by Pontryagin's Maximum Principle. The linear solutions are then used to obtain time optimal solutions for the nonlinear problem by a multiple-shooting algorithm. Although this approach is applicable to arbitrary boundary conditions, this work is confined, almost exclusively, to rest-to-rest maneuvers. These maneuvers are shown to possess some interesting symmetric and asymptotic properties. The problem is further analyzed in infinite dimensional space, and the convergence of the finitedimensional approximations is studied. Finally, a soft version of the time optimal slewing problem is considered, where the control is bounded only by a penalty term in the cost functional. A perturbation technique is applied to further simplify this probleril. 
To the memory of my parcuts 


\section{Acknowledgements}

I wish to express my sincere gratitude to my adviser and friend Dr. E. M. Cliff for his guidance, support and constant encouragement throughout the course of this research. I wish to thank the members of my dissertation committee for their time and effort expended in my behalf.

It has been a unique privilege to carry out this research under the inspiration of the late Prof. H. J. Kelley, who passed away before the completion of this work. He will always be remembered as a great teacher, scholar and human being.

I would like to thank the Israel Military Industries for helping me in my graduate studies and to all those people at Virginia Polytechnic Institute and State University who assisted in bringing this work to completion.

Finally, I wish to thank my wife. for her constant support throughout these years together. 


\section{Table of Contents}

1. Introduction 1

2. Equations of Motion $\quad 6$

$\begin{array}{ll}2.1 \text { Hamilton's Principle } & 7\end{array}$

2.2 Discretization by the Assumed Modes Method 9

$\begin{array}{ll}2.3 \text { Controllabilty of the Discrete Model } & 12\end{array}$

$\begin{array}{ll}2.4 \text { Numerical Results } & 13\end{array}$

2.5 Governing Equations for the Distributed Linear Model 14

3. Time Optimal Control for a Linear Discrete Model 17

$\begin{array}{lc}\text { 3.1 Problem Formulation } & 18\end{array}$

$\begin{array}{ll}\text { 3.2 Method of Solution } & 20\end{array}$

$\begin{array}{lr}\text { 3.3 Computational Results } & 24\end{array}$ 
4.1 Asymptotic Bchavior

4.2 Small Slew Angle Approximation $\quad 34$

5. Some Extensions $\quad 38$

5.1 Damping $\quad 39$

5.2 Nonlinear Effects $\quad 41$

5.3 Symmetric Properties of Rest-to-Rest Maneuvers 43

$\begin{array}{ll}5.3 \text { Nonideal Actuator } & 45\end{array}$

6. Problem Analysis in Infinite Dimensional Space 48

6.1 Model Formulation $\quad 49$

6.2 Exact and Approximate Controllability 53

6.3 Time Optimal Control 55

7. Time Optimal Control with Penalty on the Control Effort 59

$\begin{array}{ll}\text { 7.1 Problem Formulation } & 60\end{array}$

$\begin{array}{ll}7.2 \text { Optimality Conditions } & 61\end{array}$

$\begin{array}{ll}7.3 \text { Computational Results } & 62\end{array}$

7.4 Solution by a Singular Perturbation Technique 63

$\begin{array}{ll}\text { 8. Conclusions and Recommendations } & 67\end{array}$

$\begin{array}{ll}\text { 8.1 Summary and Conclusions } & 67\end{array}$ 
8.2 Comparison with Some Other Works 69

$\begin{array}{ll}8.3 \text { Recommendations } & 71\end{array}$

Appendix : A Brief History of the Time Optimal Control Problem 73

References $\quad 83$ 


\section{List of Figures}

Fig. 1 : Flexible Structure

Fig. 2 : Natural Frequencies 93

Fig. 3 : Natural Frequencies $\quad 94$

Fig. 4 : Rigid Body - Rest-to-Rest $\left(x^{\circ}=30\right)$

Fig. 5 : One Elastic Mode - Rest-to-Rest $\left(x^{\circ}=30\right)$

Fig. 6: Phase Space - Rest-to-Rest 97

Fig. 7 : Phase Space - Rest-to-Rest 98

Fig. 8 : Switching Points vs. Slew Angle 99

Fig. 9 : Slewing Time vs. Slew Angle 100

Fig. 10 : One Elastic Mode - Spin-Up 101

Fig. 11: Two Elastic Modes - Rest-to-Rest $\left(x^{\circ}=30\right)$

Fig. 12: Suboptimal Solution $\left(x^{\circ}=10\right) \quad 104$

Fig. 13: Optimal Solution $\left(x^{\circ}=10\right) \quad 105$

Fig. 14 : Switching Points vs. Frequency 106 
Fig. 15 : Slewing Time vs. Frequency

Fig. 16 : Approximate Solution $\left(x^{\circ}=1\right)$

Fig. 17 : Exact Solution $\left(x^{\circ}=1\right)$

Fig. 18: Damped System

Fig. 19 : Nonlinear System

Fig. 20 : Switching Points vs. Torque

Fig. 21 : Slewing Time vs. Torque

Fig. 22 : Linear solution $(p=3)$

Fig. 23 : Nonlinear solution $(p=3)$

Fig. 24 : Nonideal Actuator $(\tau=0.5)$

Fig. 25 : Nonideal Actuator $(\tau=0.1)$

Fig. 26 : Switching Points vs. $\tau$

Fig. 27 : Slewing Time vs. $\tau$

Fig. 28 : Residual Energy vs. $\tau$

Fig. 29 : Switching Function $\left(x^{\circ}=10\right)$

Fig. 30 : Switching Function $\left(x^{\circ}=30\right)$

Fig. 31 : Rigid Body - Rest-to-Rest $\left(x^{\circ}=15\right)$

Fig. 32 : One Elastic Mode - Rest-to-Rest $\left(x^{\circ}=15\right)$

Fig. 33 : Two Elastic Modes - Rest-to-Rest $\left(x^{\circ}=15\right)$

Fig. 34 : Two Elastic Modes - Rest-to-Rest (Time Fixed)

Fig. 35 : Linear System - Optimal Control Functions

Fig. 36 : Linear System - Minimum Effort Problem 
Fig. 38 : Nonlinear System - Optimal Control Functions 


\section{List of Tables}

Table 1: Natural Frequencies 16

Table 2: Terminal Time 29

Table 3 : Residual Energy $\quad 30$

Table 4 : Terminal Time for Two Flexible Modes 37

$\begin{array}{ll}\text { List of Tables } & \text { xi }\end{array}$ 


\section{Introduction}

In recent years there has been considerable interest in control for flexible structures. It is becoming increasingly important to take into consideration the flexiblity of structures in the design process of control systems for modern acrospace vehicles. Consequently, requirements for active vibration suppresion is becoming part of attitude-control-system specifications for modern aircraft and spacecraft. Single-axis slew mancuvers of simple flexible spacccraft, consisting of a rigid hub and flexible appendages, have been investigated extensively by Turner et al. [1-4] and by Breakwell [5]. The approach was based on the Linear Quadratic Regulator (LQR) theory, where the cost was a combination of control effort and system energy. Under this formulation closed-loop control is possible, and the feedback gains can be obtained in closed form (for the linearized system). Lisowski and Hale [6] extended these results by freeing structural parameters in the optimization process, so that both structural parameters and active control torques can be determined in order to minimize a specific cost functional. Exper- 
imental results for LQR design have been reported by Juang, Horta, and Robertshaw [7]. The measurements were compared with analytical predictions, and sufficient agreement has been obtained. Skaar et al. [8] studied a satellite reorientation problem using thrusters rather than internal momentum transfer devices. The control is on-off in open loop. The cost was the post-maneuver elastic energy, and optimal switching points were obtained to minimize it.

A problem of current interest is to include the transition time in the cost in order to accomplish fast slew maneuvers. Vander Velde and He [9] investigated a problem of this kind where the cost is a combination of fuel and time. The solution again is open-loop on-off control of the spacecraft thrusters. Thompson et al. [10] considered near time-optimal controls by minimizing a sum of the time and a certain measure of residual energy. The solution is open loop and is continuous with respect to time. Meirovitch et al. [11,12] explored the idea of controlling the rigid body by time optimal controls, while considering the elastic mode control as a modified LQR problem. In this formulation, the rigid-body time optimal solution is a forcing (disturbance) term in the elastic equations. Closed-loop solutions are sought in order to suppress the induced vibrations.

The purpose of this work is to study the pure time-optimal slewing problem where the cost is the transition time only. This problem is currently being investigated and first results have been reported [13-16]. The major differences between these works are. in the computational methods. These being a direct two-point-boundary-valuc-problem approach in [13], a gradient method in [14], a homotopy method in [15] and a combination of a parameter optimization tech- 
nique and a Multiple Shooting Algorithm (MSA) in [16] (which contains preliminary results from this work). There is, however, a qualitative agreement in the solutions of the time optimal control problcm. It secms natural to ask why such a variety of methods have been applied to solve almost the same time optimal control problem.

The minimum-time control problem has a long history, from the Bernoulli brothers in the seventeenth century to our very days. (See Appendix for a short history of the problem.) The theoretical knowledge about the linear time-optimal control problem in finite dimensional space is complete. The single-axis slewing problem for a flexible spacecraft is, however, a problem in infinite dimensional space and it contains some nonlinear terms. It is also computationally nontrivial in linear discrete space due to the complex eigenvalues of the flexible structure. The various methods differ in the way they handle these difficulties.

There are, accordingly, three main objectives to this work. First to construct a simple algorithm to solve the finite-dimensional linear problem. It is, however, desirable to make it more easily generalized than those employed in [13-15]. The second objective is to extend the results to include the system nonlinearities. This has been ignored in [13] and [15] and has been considered in [14] by a very complicated numerical scheme. The third objective is to study the problem in infinite dimensional Hilbert space which has not been done elswhere for this system. The following chapters are organized accordingly. Chapter 2 contains a derivation by Hamilton's principle and the assumed-modes methods of the different models that are used in rest of the work. In chapter 3 the linear time-optimal slewing 
problem is explored. A novel parameter optimization technique is presented along with numerous computational results, primarily for rest-to-rest maneuvers, that have been obtained by this approach. This is continued in chapter 4 where certain approximations are suggested as a result of some interesting asymptotic properties of the solutions. The effects of nonlinearity, along with other extensions, are studied in chapter 5 . This chapter presents numerical solutions obtained by a two-point boundary-value problem solver which employs a multiple-shooting algorithm and uses the linear solutions as initial guesses. Interesting symmetry properties are shown to be possessed by both the linear and the nonlinear problems (for rest-to-rest maneuvers). Chapter 6 presents an analysis of the problem in infinite dimensional space and revisits the results of Chapter 3. It contains three parts. The first part is a state-space formulation of the system in Hilbert space, the second, a discussion of controllability, and the third, a study of the time-optimal control problem and of the convergence properties of the discrete solutions. Chapter 7 is somewhat out-of-line with respect to the previous chapters. The problem of soft-constrained time-optimal control is studied where the cost is a combination of control effort and transition time. A singular-perturbation technique is introduced to reduce the computational effort. In chapter 8 conclusions are drawn from this work, and from the previouslymentioned parallel works which have been studying the time-optimal slewing problem. We also offer some recommendations for further study. The appendix covers bricfly the history of the time-optimal control theory, and is concentrated mainly on the development of ideas and concepts rather than specific applica- 
tions. It emphasizes aspects which are of importance to this work but it is not restricted to them. 


\section{Equations of Motion}

The mathematical model for the structure shown in Figure 1 is developed in this chapter.

A single axis rotation is considered for a system consisting of a rigid hub with an elastic appendage attached to it. The appendage is modeled as an EulerBernoulli cantilevered beam. The system is controlled by a single actuator which exerts an external torque on the rigid hub.

Hamilton's Principle is applied to derive the equations of motion. The assumed-modes method is applied to obtain a finite dimensional system of ordinary differential equations. The finite dimensional model will be used later for all of the numerical computations. For the linearized system the form of partial differential equations shall be retained for an analysis of the problem in infinite dimensional space. 


\subsection{Hamilton's Principle}

The kinetic energy of the system is (Fig. 1)

$T=\frac{1}{2} \int \dot{\bar{r}} T \dot{\bar{r}} d m+\frac{1}{2} I_{h} \dot{\alpha}^{2}$

where $I_{h}$ is the hub moment-of-inertia, $\alpha(\mathrm{t})$ is the angular displacement of the hub, $\mathrm{m}$ is the mass distribution of the beam, $l$ is the beam length, $r(x, t)$ is the radius vector $x \bar{i}+w(x, t) \bar{j}$, where $\mathrm{w}(\mathrm{x}, \mathrm{t})$ is the elastic deflection of the beam.

Therefore, the inertial velocity is given by

$\dot{\bar{r}}=(-\dot{\alpha} w) \bar{i}+(\dot{\alpha} x+\dot{w}) \bar{j}$

Substituting (2.2) into (2.1) we obtain

$T=\frac{1}{2} \int_{0}^{l}\left(\dot{\alpha}^{2}\left(x^{2}+w^{2}\right)+\dot{w}^{2}+2 \dot{\alpha} \dot{w} x\right) m d x+\frac{1}{2} I_{h} \dot{\alpha}^{2}$.

The potential encrgy is

$V=\frac{1}{2} \int_{0}^{l} E I\left(w^{\prime \prime}\right)^{2} d x+\int_{0}^{l} F(x)(d s-d x)$ 
where $E I$ is the beam rigidity and ()$^{\prime}$ denotes derivative with respect to $x, F(x)$ denotes the axial force, which arises form centrifugal effects, ds is an increment of length along the displaced axis and $\mathrm{dx}$ is its projection. (See [17, p. 235] for a similar derivation.)

Calculating $\mathrm{F}(\mathrm{x})$

$F(x)=\int_{x}^{l} m \dot{\alpha}^{2} \zeta d \zeta=\frac{m \dot{\alpha}^{2}}{2}\left(l^{2}-x^{2}\right)$.

We assume that the shortening of the projection $(d s-d x)$ in $(2.4)$ can be approximated by

$d s-d x \sim \frac{1}{2} w^{\prime 2} d x$

hence

$V=\frac{1}{2} \int_{0}^{l} E I\left(w^{\prime \prime}\right)^{2} d x+\int_{0}^{l} \frac{m \dot{\alpha}^{2}}{4}\left(l^{2}-x^{2}\right) w^{\prime 2} d x$.

The virtual work done by the control torque $u$ is

$\delta W=u \delta \alpha$.

The equations of motion can now be obtained from Hamilton's Principle 


$$
\int_{0}^{t}(\delta T-\delta V+\delta W) d t=0
$$

2.2 Discretization by the Assumed Modes Method

Assume [17]

$$
w(x, t)=\sum_{i=1}^{k} \eta_{i}(t) \Psi_{i}(x)
$$

where $\left\{\Psi_{i}\right\}$ is a complete set of admissible functions (i.e. functions which satisfy the geometric boundary conditions but not necessarily the natural boundary conditions).

The kinetic and potential energies are

$$
\begin{aligned}
& T=\frac{1}{2} \dot{\alpha}^{2} \eta^{T} M \eta+\frac{1}{2} \dot{\eta}^{T} M \dot{\eta}+\dot{\alpha} Q^{T} \dot{\eta}+\frac{1}{2} I \dot{\alpha}^{2}, \\
& V=\frac{1}{2} \eta^{T} K \eta+\frac{1}{2} \dot{\alpha}^{2} \eta^{T} R \eta .
\end{aligned}
$$

Where $\eta$ is a k-dimensional column vector with entrics $\eta_{i}$, and where

$$
\left[M_{i, j}\right]=\int_{0}^{l} m \Psi_{i} \Psi_{j} d x,
$$


$\left[K_{i, j}\right]=\int_{0}^{l} E \Gamma \Psi_{i}^{\prime \prime} \Psi_{j}^{\prime \prime} d x$

$\left[R_{i, j}\right]=\int_{0}^{l} m \frac{\left(l^{2}-x^{2}\right)}{2} \Psi_{i}^{\prime} \Psi_{j}^{\prime} d x$

$\left[Q_{i}\right]=\int_{0}^{l} m x \Psi_{i} d x$

$I=I_{h}+\int_{0}^{l} m x^{2} d x$

Let $\mathrm{L}$ be The Lagrangian $\mathrm{L}=\mathrm{T}-\mathrm{V}$. Lagrange's equations of motion are

$\frac{d}{d t} \frac{\partial L}{\partial \dot{\alpha}}-\frac{\partial L}{\partial \alpha}=u$

$\frac{d}{d t} \frac{\partial L}{\partial \dot{\eta}}-\frac{\partial L}{\partial \eta}=0$

Hence

$I \ddot{\alpha}+Q^{T} \ddot{\eta}+2 \dot{\eta}^{T}(M-R) \eta \dot{\alpha}+\eta^{T}(M-R) \eta \ddot{\alpha}=u$

$Q \ddot{\alpha}+M \ddot{\eta}-\left[\dot{\alpha}^{2}(M-R) \dot{-} K\right] \eta=0$.

Neglecting the higher order terms, a linear model can be obtained 
$I \ddot{\alpha}+Q^{T} \ddot{\eta}=u$,

$Q \ddot{\alpha}+M \ddot{\eta}+K \eta=0$.

It is suggested in [1] to retain, for nonlinear modelling of the system, the $\dot{\alpha}^{2}$ term and to neglect the rest of the nonlinear terms. Notice that by so doing only small deflections of the beam are considered, with possibly high angular rigid body rates. In this case equations (2.16) and (2.17) will be the governing equations.

We shall first deal with the linear model. Let

$$
\begin{aligned}
& \tilde{K}=\left[\begin{array}{ll}
0 & 0 \\
0 & K
\end{array}\right], \\
& \tilde{M}=\left[\begin{array}{ll}
I & Q^{T} \\
Q & M
\end{array}\right] .
\end{aligned}
$$

We solve the eigenvalue problem

$$
\omega_{j}^{2} \tilde{M} v_{j}=\tilde{K} v_{j} \quad j=0,1,2, \ldots k
$$

Let $E$ be the modal matrix (the column $\mathrm{j}$ of which is the $\mathrm{j}$-th eigenvector $v_{j}$ ), $\Omega^{2}$ be a diagonal matrix with entries $\omega_{j}^{2}$ (the eigenvalues) and $G$ be the transpose of the first row of $\mathrm{E}$ with entries $g_{j}$.

Let $\zeta$ be defined by

$$
E \zeta=\left[\begin{array}{l}
\alpha \\
\eta
\end{array}\right]
$$


then, from (2.17) and (2.18), the linear equations of motion become

$\ddot{\zeta}+\Omega^{2} \zeta=G u$

In order to obtain a nonlinear model we define [1]

$D=E^{T}\left[\begin{array}{rr}0 & 0 \\ 0 & R-M\end{array}\right] E$

thus, from (2.16) and (2.17), the nonlinear equations of motion become

$\ddot{\zeta}+\Omega^{2} \zeta=G u-\left(G^{T} \dot{\zeta}\right)^{2} D \zeta$

\subsection{Controllabilty of the Discrete System}

A necessary and sufficient condition for controllabilty of the system (2.22) is that the eigenvalues are distinct and that every entry of the control-influence vector $\mathrm{G}$ is nonzero [18].

Consider the eigenvalue problem of the beam

$\bar{\omega}_{j}^{2} M \bar{v}_{j}=K \bar{v}_{j} \quad j=1,2, \ldots k$

where the matrices $\mathrm{K}$ and $\mathrm{M}$ are given by (2.12.1) and (2.12.2). By the Inclusion Principle $[17, \mathrm{p} .292]$ the eigenvalues of this problem satisfy

$\omega_{j-1}^{2} \leq \bar{\omega}_{j}^{2} \leq \omega_{j}^{2} \quad j=1,2, \ldots k$ 
Notice that $\omega_{0}$ is zero (the rigid body mode). Assume now that these inequalities are satisfied in the strong sense. We will show that this assumption entails controllabilty. First, with strong inequalities in (2.26), it is clear that the eigenvalues $\omega_{0}^{2}, \omega_{1}^{2}, \ldots \omega_{k}^{2}$ are distinct. For the second requirement, notice that if an entry $g_{j}$ is zero, then the associated eigenvector $v_{j}$ has a zero in the first entry. Denote this vector by

$v_{j}=\left[\begin{array}{l}0 \\ \bar{v}_{j}\end{array}\right]$.

Clearly $\bar{v}_{j}$ is an eigenvector of $(2.25)$ with eigenvalue $\bar{\omega}_{j}^{2}=\omega_{j}^{2}$ contradictory to our assumption. Therefore the system is controllable.

\subsection{Numerical Results}

Values for the system parameters have been selected to be appropriate for an aluminium beam that is $30 \mathrm{ft}$ long and has a rectangular cross-section with height $1 \mathrm{ft}$ and thickness $.25 \mathrm{in}$. These are representative values for a solar pancl [19]. The system parameters are:

Hub moment of inertia $=981\left[\right.$ slug $\left.-f t^{2}\right]$

Bcam rigidity $(\mathrm{EI}) \quad=3.49 \times 10^{4}\left[l b f-f t^{2}\right]$

Beam length $(\mathrm{l}) \quad=30[\mathrm{ft}]$

Beam mass distribution $(\mathrm{m})=0.109[$ slug/ft $]$.

Following [1] the set of admissible functions is chosen to be 
$\Psi_{j}(x)=\left(\frac{x}{l}\right)^{j+1} \quad j=1,2, \ldots$

It is important to note that, with this choice, the mass matrix is an ill-conditioned Hilbert matrix and therefore numerical problems might show up if roundoff errors are not kept sufficiently small. The resulting natural frequencies are given in Table 1 for a varied number of assumed modes. The first two nonzero frequencies are also presented in Fig. 2.

It is of interest to compare these results with the associated values of the unrotating cantilevered beam. Fig. 3 presents the behavior of the first two nonzero frequencies as the ratio between the total moment of inertia (I) and the beam moment of inertia (IB $\left.=I-I_{h}\right)$ is increased. Five modes have been used to approximate the deflections. The fixed cantilcvered beam is the limit case when the hub moment of inertia approaches infinity. The first two analytical values for the cantilevered case are $\omega_{1}=2.211 \ldots, \omega_{2}=13.854 \ldots$, and the natural frequencies are converging to these values from above.

\subsection{Governing Equations For the Distributed Linear Model}

Neglecting high order terms in the cnergy equations (2.3) and (2.4), the energies become 
$T=\frac{1}{2} \int_{0}^{l}\left(\dot{\alpha}^{2} x^{2}+\dot{w}^{2}+2 \dot{\alpha} \dot{w} x\right) m d x+\frac{1}{2} I_{h} \dot{\alpha}^{2}$,

$V=\frac{1}{2} \int_{0}^{l} E I w^{\prime \prime 2} d x$

Applying Hamilton's Principle (equation (2.9)), the governing equations can be obtained

$m(\ddot{w}+x \ddot{x})+\left(E I w^{\prime \prime}\right)^{\prime \prime}=0$,

$I \ddot{\alpha}+\int_{0}^{l} \ddot{w} m x d x=u$

along with the boundary conditions

$w(0, t)=w^{\prime}(0, t)=w^{\prime \prime}(l, t)=w^{\prime \prime \prime}(l, t)=0$.

Substituting $\ddot{w}$ from (2.30) into (2.31) and integrating by parts, an alternative form for (2.31) is obtained

$I_{h} \ddot{\alpha}-E I w^{\prime \prime}(0, t)=u$. 
Table 1: Natural Frequencies

$$
\left[\frac{1}{\sec }\right]
$$

Dimension

of

\begin{tabular}{c|c|c|c|c|c} 
Approximation & $\omega_{0}$ & $\omega_{1}$ & $\omega_{2}$ & $\omega_{3}$ & $\omega_{4}$ \\
\hline 1 & 0 & - & - & - & - \\
\hline 2 & 0 & 3.857 & - & - & - \\
\hline 3 & 0 & 3.089 & 22.135 & - & - \\
\hline 4 & 0 & 3.081 & 14.147 & 74.380 & - \\
\hline 5 & 0 & 3.080 & 14.102 & 39.864 & 56.20 \\
\hline
\end{tabular}




\section{Time Optimal Control for a Linear Discrete}

\section{Model}

In this chapter the time-optimal slewing problem is considered for a spatially discretized linear system. Nonlinear effects, the effect of structural damping and nonideal actuators are not considered in this chapter, but will be examined in subsequent chapters. The problem is investigated in modal space in which a reduced model is used for the control design.

The time-optimal problem for finite dimensional linear systems has been thoroughly investigated in the past forty years, and the theory today is quite complete. For the system under consideration the optimal control is bang-bang, and our main objective is to understand the switching structure.

A technique is developed to obtain the switching structure by parameter optimization and to verify the optimality of this solution by the Maximum Principle [20] without having to solve the two-point boundary-value problem. Although the technique is applicable to arbitrary boundary conditions, the discussion is 
confined, almost exclusively, to rest-to-rest mancuvers which are of practical importance. For the numerical examples given below, five assumed-modes are used to discretize the sysetm.

\subsection{Problem Formulation}

Consider the linear equations of motion (2.22)

$\ddot{\zeta}+\Omega^{2} \zeta=G u$

Define

$\bar{x}=\left[x_{1}, x_{2}, \ldots x_{n}\right]^{T}$

where

$x_{2 i-1}=\zeta_{i} \quad, \quad x_{2 i}=\dot{\zeta}_{i}, \quad i=1,2 \ldots$

A linear state space model is obtained

$\dot{\bar{x}}(t)=A \bar{x}(t)+b u(t)$

where 
$A=\left[\begin{array}{ccccccc}0 & 1 & & & & \\ 0 & 0 & & & & \\ & & 0 & 1 & & \\ & & -\omega_{1}^{2} & 0 & & & \\ & & & & \vdots & & \\ & & & & 0 & 1 \\ & & & & -\omega_{k}^{2} & 0\end{array}\right]$

$b=\left[\begin{array}{lllllllll}0 & g_{0} & 0 & g_{1} & \ldots & 0 & g_{k}\end{array}\right]^{T}$.

Note that $k$ is the number of flexible modes and $n=2 k+2$ is the dimension of the system.

The time-optimal problem is to find a measurable function $u$ which drives in minimum time the state $\bar{x}$ from a given value

$\bar{x}(0)=x_{0}$

to a target set

$\bar{x}\left(t_{f}\right) \Theta \Theta$

subject to

$|u(t)| \leq u_{m} \quad ; \quad 0 \leq t \leq t_{f}$

where $u_{m}$ is the maximum admissible torque. 


\subsection{Method of Solution}

The system (3.3) is linear, marginally stable, and controllable. In the terminology of LaSalle [20] the system is also normal as a result from the one-dimensionality of the control space (see appendix). Existence and uniqueness of the optimal solution are guaranteed, and the Maximum Principle is a sufficient as well as necessary condition for it. Singular subarcs can also be ruled out by normality [20].

Let $\tilde{u}$ be the optimal control. The Maximum Principle asserts the existence of a nontrivial solution $\lambda(t)$ for the adjoint equation

$\dot{\lambda}(t)=-A^{T} \lambda(t)$

Where $\lambda\left(t_{f}\right)$ is orthogonal to $\Theta$, and

$\tilde{u}(t)=-u_{m} \operatorname{sgn}\left(\dot{\lambda}^{T}(t) b\right)$

Thus, the optimal control is bang-bang and governed by a switching function $\lambda^{T} b$. The number of switching points is, however, unknown and, unlike the case when $\mathrm{A}$ has real distinct eigenvalues, we do not have, in gencral, an upper limit for it. At each switching point the switching function must vanish.

We consider now the important case of rest-to-rest maneuvers, to a target point $x_{f}$ (typically the origin). The solution to the problem can be obtained by parameter optimization. We formulate the following problem. 
Let $\left\{t_{1}, t_{2}, \ldots . t_{m}, t_{f}\right\}$ i.e. $m$ switching points and the final time, be free parameters.

Minimize the cost

$J=t_{f}$

subject to

$u_{m} h_{i}\left(t_{f}\right)+2 u_{m} \sum_{j=1}^{m}(-1)^{\dot{\gamma}} h_{i}\left(t_{f}-t_{j}\right)=x_{f_{i}}-x_{o_{i} \cdot} \quad i=1,2 \ldots n$

where $h_{i}(t)$ are simple unit-step response functions. By the superposition principle, we consider the bang-bang control function as a linear combination of $m+1$ step functions. The first step starts at $\mathrm{t}=0$, with amplitude $u_{m}$, and the rest take place at the switching points, with altcrnating signs, and with amplitude $2 u_{m}$. For the rigid body mode

$h_{1}(t)=0.5\left(g_{0}\right) t^{2}$

and

$h_{2}(t)=\left(g_{0}\right) t$

For the elastic modes

$h_{2 i-1}(t)=\omega_{i}^{-2}\left(g_{i}\right)\left(1-\cos \omega_{i} t\right)$ 
and

$h_{2 i}(t)=\omega_{i}^{-1}\left(g_{i}\right) \sin \omega_{i} t$

Notice that both the constraints and the cost are analytically differentiable with respect to each parameter. This fact is very important in parameter optimization, since gradient methods work with sensitivity derivatives of the cost and the constraints, and these derivatives are evaluated, in general, at each iteration step. Since the number of switchings is not known, and for any given number of parameters an optimal set may exist, we need to verify that the solution obtained via parameter optimization is, indeed, the optimal solution. Recall that the optimal control problem is guaranteed to have a unique solution.

The adjoint vector $\lambda(t)$ is by the Maximum Principle

$\lambda(t)=e^{-A^{T} t} \lambda(0)$

Hence the switching function is

$b^{T} \lambda(t)=b^{T} e^{-A^{T} t} \lambda(0)$

For each switching point $t_{i}, \quad i=1,2, \ldots m$ we obtain

$b^{T} e^{-A^{T} t_{i}} \lambda(0)=0$

Construct the $m \times n$ matrix $\mathrm{P}$ 


$$
P=\left[\begin{array}{c}
b^{T} e^{-A^{T} t_{1}} \\
b^{T} e^{-A^{T} t_{2}} \\
\vdots \\
b^{T} e^{-A^{T} t_{m}}
\end{array}\right]
$$

to obtain

$$
P \lambda(0)=0
$$

We make the following observations:

1. $\lambda(0)$ is in the null space of $P$.

2. The nullity of $P$ satisfies $: \operatorname{dim}($ null $P)=n-\operatorname{rank} P$.

3. By the Maximum Principle there exists a nontrivial solution to the homogenous adjoint equations. Therefore, if the solution obtained by parameter optimization is the optimal solution, then null $\mathrm{P}$ is not empty.

4. If the nullity is greater than 1 , we may be able to use the transversality condition for further reducing this subspace by intersecting the two subspaces.

5. Knowing $\lambda(0)$ the Maximum Principle can be verified by directly evaluating $\lambda(t)$

6. We can use $\lambda(0)$ of the linear problem as a starting point (along with the switching points) for the two-point boundary-value problem associated with the nonlinear model. 


\subsection{Computational Results}

We consider rest-to-rest maneuvers where the system is to be driven to $\bar{x}\left(t_{f}\right)=0$ from

$\bar{x}(0)=\left[-x^{0}, 0,0,0 \ldots . . .\right]^{T}$

The time-optimal solution for the rigid body problem is very well known [20]. The solution for $x^{\circ}=30$ and $u_{m}=221.4 \mathrm{lb}$ - $\mathrm{ft}$ is shown in Fig. 4 where the elapsed time is normalized by $t_{f}$ (To find the actual slew angle in radians one has to multipy $x^{\circ}$ by $g_{0}$ which, for our example, is 0.0226 slug $^{-1} f t^{-2}$. Thus, this example corresponds to $0.678 \mathrm{rad}$ or $38.8 \mathrm{deg}$.)

There is a single switching at the midpoint, and the terminal time is

$t_{f_{r}}=2 \sqrt{\frac{x^{o}}{g_{0} u_{m}}}=4.90 \mathrm{sec}$.

Fig. 5 presents, in normalized time, a solution for the problem (for $x^{\circ}$ and $u_{m}$ as above), where the first elastic mode $\left(\omega_{1}=3.08 \mathrm{sec}^{-1}\right.$ and $g_{1}=0.0219$ slug $\left.^{-1} f^{-2}\right)$ is taken into account. Solutions for the parameter optimization problem have been obtained by an optimization program which employs an iterative procedure based on a variable-metric gradient-projection algorithm [21]. It turns out that there are three switching points: the original one at the midpoint, and two others symmetricaily located before and after the middle point. The terminal time is $5.00 \mathrm{sec}$. The switching function, obtained by the null space technique, is also presented to verify the optimality of the solution. It is noted that for some initial estimates 
of the time parameters the optimization procedure found other solutions with three switching points that failed to satisfy the Maximum Principle.

Fig. 6 and Fig. 7 present the results in a more familiar phase space. The trajectories are parabolas in $\left(x_{1}, x_{2}\right)$ plane, and ellipses in $\left(x_{3}, x_{4}\right)$ plane.

As we vary the slew angle, the switching at the midpoint (in normalized time) remains fixed, but the other two move as shown in Fig. 8. Fig. 9 shows the slewing time versus the slew angle for the problem. Notice that at certain points the solution coincides with the rigid body solution. From Fig. 8 we conclude that at these points there is, indeed, only one switching point (i.e. the rigid body solution). To explain this phenomenon one may evaluate the response of the onemode elastic equation to the rigid body optimal control function to obtain

$x_{3}\left(t_{f}\right)=-2\left(g_{1} u_{m}\right) \omega^{-2} \cos \left(\frac{\omega t_{f}}{2}\right) \sin ^{2}\left(\frac{\omega t_{f}}{4}\right)$

$x_{4}\left(t_{f}\right)=-2\left(g_{1} u_{m}\right) \omega^{-1} \sin \left(\frac{\omega t_{f}}{2}\right) \sin ^{2}\left(\frac{\omega t_{f}}{4}\right)$.

Hence, in agreement with the numcrical results, for countablly many points which satisfy

$\frac{\omega t_{f}}{4}=p \pi \quad p=0,1,2, \ldots$

the elastic boundary conditions are satisfied by the rigid-body optimal control so that the solution contains only one switching point. The matrix $P$ is therefore [1x4] with nullity 3 . This is an example for a nonunique $\lambda(t)$ which determines a 
unique optimal control. As pointed out by [22], uniqueness of the control and its being determined uniquely by the necessary condition have nothing to do with the uniqueness of the direction $\lambda$ (p. 64). For most cases, however, three switching points are required. From the numerical results, we conclude that for rest-to-rest maneuvers, with one elastic mode, the matrix $P$ is a [3x4] matrix with onedimensional null space (almost always). The initial adjoint vector is therefore uniquely determined by the switching structure.

In order to demonstrate a different situation we consider a minimum time spin-up maneuver. The problem is to drive the system from the origin to

$x\left(t_{f}\right)=[\theta, \Omega, 0,0]$

where $\Omega$ is given, $\theta$ is free, and the cost to be minimized is again, the terminal time. Notice that the target set $\Theta$ is now the entire $x_{1}$ axis, rather than a single point. The method of parameter optimization however is still applicable. The solution for the rigid body equivalent problem is, of course, a constant maximum control torque. The solution for the elastic case (one flexible mode) is shown in Fig. 10. There are two switching points, and the symmetry is preserved. $P$ however, is a [2x4] matrix of rank 2 (and hence nullity 2). Based on the transversality conditions, $\lambda\left(t_{f}\right)$ should be orthogonal to $\Theta$, hence $\lambda_{1}(t)$ (which is constant by the adjoint equations) is zero. This defines three-dimensional subspace, the intersection of which with null(P) yiclds the desired $\lambda(0)$, and the Maximum Principle can be verified. The switching function is also presented in Fig. 10. 
We shall return now to rest-to-rest maneuvers to include the first two elastic modes in our control design. Fig. 11 presents the solution for our test case $x^{\circ}=30\left(\omega_{2}=14.1 \mathrm{sec}^{-1}\right.$ and $g_{2}=-0.0051$ slug $\left.^{-1} f t^{-2}\right)$. The total time is only 0.004 sec more than the one elastic mode case. The number of switching points is five, the symmetry is preserved, and the switching points seem to be small perturbations of the one flexible mode case where the two new switching points take place near the midpoint. Loosely spcaking, in some integral sense, we may argue that the process is converging. We shall come back to the convergence problem later in chapter 6. An interesting question is whether the number of switching points for rest-to-rest maneuvers with two flexible modes is always five or less (and therefore is consistent with the real eigenvalue cases [20]). To answer this question a different slewing angle is considered. Fig. 12 presents the optimal five switching points for $x^{\circ}=10$, the terminal time is very close to the one flexible mode solution but the Maximum Principle is not satisfied! Further investigation reveals a different solution made of seven switching points - Fig. 13 - which satisfies the Maximum Principle, and yields a slightly better transition time. It is noted that the optimal solution is still a perturbation to the one flexible mode with two more pulses created by the new switching points.

The residual energy (the energy stored in the uncontrolled modes) for the test case of Fig. 4, 5, 8 was calculated using the five assumed modes. The energy is $5.08 l b f-f t$ for the rigid body solution, $0.07 l b f-f t$ for the one flexible mode, and $0.009 \mathrm{lbf}-f t$ for the two flexible modes. Thercfore, for this case, the error obtained by controlling only the fundamental frequency is relativly very small. 
Two more cases have been studied in which we consider the beam rigidity EI to be $10 \%$ and $2 \%$ of its nominal value. Table 2 and Table 3 summarize the results for the cost (the terminal time) and the residual energy, respectively. It turns out that the first flexible mode is still dominant in determining the terminal time. However, the residual energy may not be negligble under one-flexible-mode control. Consequently, for certain applications, more controlled modes may be required.

The symmetry of the switching-point structure has been preserved in all cases. We shall postpone the discussion of this interesting property to chapter 5 where the damped and the nonlinear cases will be studied. In the meanwhile, we shall present some interesting asymptotic properties of the solutions. 
Table 2: Terminal Time

[sec]

EI (nom) $0.1 \mathrm{EI} \quad 0.02 \mathrm{EI}$

\begin{tabular}{l|c|c|c}
\hline rigid body control & 4.90 & 4.90 & 4.90 \\
\hline 1 -mode control & 5.000 & 6.69 & 10.14 \\
\hline 2 -mode control & 5.004 & 6.71 & 10.41 \\
\hline
\end{tabular}


Table 3: Residual Energy

[ $1 b-f t$ ]

\begin{tabular}{l|c|c|c}
\multicolumn{2}{c}{ EI } & 0.1 EI & 0.02 EI \\
\hline rigid body control & 5.08 & 293.5 & 136.2 \\
\hline 1 -mode control & 0.07 & 0.243 & 4.047 \\
\hline 2 -mode control & 0.009 & 0.002 & 0.032 \\
\hline
\end{tabular}




\section{Asymptotic Behavior and Approximations}

In this chapter we continue to investigate the time-optimal problem for the finite dimensional model with the assumptions of the previous chapter. Based on the previously given results, it is first assumed that the system response can be adequately approximated by its rigid body mode and fundamental frequency mode. A parametric study is carried out where the stiffness, represented by the fundamental frequency, is taken to be a parameter. The asymptotic behavior of the system with respect to this parameter is studied. The effect of the second mode is then considered, by fixing the fundamental frequency while varying the sccond-elastic-mode frequency. Bascd on this study, an approximation scheme is suggested for small slew angles by which the optimal control is given in closedform expressions. 


\subsection{Asymptotic Behavior}

Consider rest-to-rest minimum-time slewing maneuvers with one elastic mode. The objective is to investigate the behavior of the solution as the flexiblity of the system is changed by varying $\omega$. The basic structure of the solution, i.e. switching at $\left\{t_{f} / 2-\Delta, t_{f} / 2, t_{f} / 2+\Delta\right\}$, is not affected by $\omega$. The value of $\Delta$ however, varies with $\omega$ as shown in Fig. 14 (normalized to $t_{j}$ ), for three different slew angles. There are two asymptotes. For high $\omega, \Delta$ approaches zero, i.e. the rigid body solution, while for low $\omega ; \bar{\Delta} \equiv \Delta / t_{f}$ approaches $(1 / \sqrt{8})$. To understand this behavior consider a zeroth order approximation for the elastic equation. As $\omega \rightarrow 0$ the step response of the elastic deflection $h_{3}(\tau) \rightarrow\left(g_{1} u_{m}\right) \tau^{2} / 2$, and the terminal value is by superposition

$x_{3}\left(t_{f}\right) \rightarrow\left(g_{1} u_{m}\right)\left(1 / 4-2 \bar{\Delta}^{2}\right) t_{f}^{2}$

Since the system is driven to the origin it is clear that $\bar{\Delta} \rightarrow(1 / \sqrt{8})$.

The rigid body response is for any $\omega$

$x_{1}\left(t_{f}\right)=-x^{o}+\left(g_{0} u_{m}\right)\left(1 / 4-2 \bar{\Delta}^{2}\right) t_{f}^{2}$

Therefore, $t_{f} \rightarrow \infty$ as $\omega \rightarrow 0$. (Recall that when $\omega=0$, the system is not controllable.)

The behavior of the terminal time with $\omega$ is shown in Fig. 15 on a logarithmic scale. Again, there are two asymptotes. For high $\omega$ the terminal time is the rigid body problem optimal time $t_{f}$, while for low $\omega$ the asymptotic line is 
$\omega t_{f}^{2}=$ constant

To explain this behavior we make a Taylor expansion for the elastic step response function

$\cos \omega \tau=1-\frac{\omega^{2} \tau^{2}}{2}+\frac{\omega^{4} \tau^{4}}{24}+\ldots$

Taking the first three items in the expansion we obtain

$\omega^{-2}\left(g_{1} u_{m}\right)(1-\cos \omega \tau) \sim\left(g_{1} u_{m}\right)\left(\frac{\tau^{2}}{2}-\frac{\omega^{2} \tau^{4}}{24}\right)$

Under this approximation the terminal elastic deflection for $\omega \rightarrow 0$ is by superposition

$x_{3}\left(t_{f}\right) \rightarrow x_{0} \frac{g_{1}}{g_{0}}-\left(g_{1} u_{m}\right) \frac{\omega^{2} t_{f}^{4}}{384}$

hence

$\omega t_{f}^{2} \rightarrow \sqrt{\frac{384 x^{0}}{\left(g_{0} u_{m}\right)}}$

which is in agreement with the numerical results. The break point of the two asymptotes is by a simple calculation

$\omega_{b}=\frac{\sqrt{96}}{t_{f_{r}}}$. 
Note that (from equation (3.20)) $t_{f_{r}}$ depends on system paramters $g_{0}, u_{m}$ and on the initial value $x^{\circ}$. An interesting fact that comes up from the optimization results, and could not be predicted by the asymptotic analysis alone, is that the asymptotes provide a very good estimate for $t_{f}$ over the entire domain of $\omega$.

We study now the effect of the second mode on this value by fixing the fundamental frequency to its nominal value $\left(3.08 \mathrm{sec}^{-1}\right)$ while varying the second nonzero natural frequency. Table 4 presents the resulting costs for $x^{\circ}=10$, where $t_{t_{1}}=3.00$ is the slewing time when only one flexible mode is considered and $t_{\delta_{2}}$ is the minimum time when two flexible modes are considered. It turns out the for $\frac{\omega_{2}}{\omega_{1}}>2$ the effect of the second mode on the slewing time is less then $0.5 \%$.

We note that the switching structure is also affected by this ratio. For small values of $\frac{\omega_{2}}{\omega_{1}}$ we have obtained five switching points, while for high values the number of switches is seven, as in Fig. 13.

\subsection{Small Slew Angle Approximation}

As already indicated, the number of controlled modes is ultimately determined by the particular application. The asymptotic analysis of the previous section provides a very simple approximation to the optimal control for small slew angles, when the one-flexible-mode control is sufficient. Notice that the approximated step response (4.5) is valid when the argument $\omega \tau$ is small. This may be the case if either $\omega$ is small or $\tau$ is small. Hence, in a given structure, this approximation 
is valid for small slew angles where $\omega$ is fixed but the transition time is small. We pursue this idea in this section.

The first step is to find out whether or not a given slew angle is considered small. A criterion for that might be

$\omega<\omega_{b}$

where $\omega_{b}$ is the break point (a function of the slew angle). If this is the case we can approximate the slewing time by

$t_{f}=\omega^{-\frac{1}{2}}\left(\frac{384 x_{o}}{g_{0} u_{m}}\right)^{\frac{1}{4}}$

An approximation for the switching points is given by

$$
\bar{\Delta}=\left(\frac{1}{8}-\frac{x_{0}}{2 g_{0} u_{m} t_{f}^{2}}\right)^{\frac{1}{2}} .
$$

Thus, we have obtained an approximation for the optimal control.

For example, let $x_{0}=1$. (For our system $\omega=3.08 \mathrm{sec}^{-1}, g_{0} u_{m}=5.0 \mathrm{sec}^{-2}$.)

Therefore

$$
\begin{aligned}
& t_{f_{r}}=2 \times\left(\frac{1}{5}\right)^{0.5}=0.894 \mathrm{sec} \\
& \omega_{b}=\frac{\sqrt{96}}{0.894}=10.9 \mathrm{sec}^{-1} .
\end{aligned}
$$

Since the criterion (4.9) is satisfied we may approximate 
$\omega t_{f}^{2}=\frac{384 \times 1}{5.0}=8.76 \mathrm{sec}$.

Thus $t_{f}=1.69 \mathrm{sec}$, and

$\bar{\Delta}=\sqrt{\frac{1}{8}-\frac{1}{2 \times 5.0 \times 1.684}}=0.3$.

The switching points are, therefore,

$\{0.337, \quad 0.8441 .350\}$.

The optimization code yields $t_{f}=1.613 \mathrm{sec}$ and the switchings

$\{0.331,0.8051 .279\}$.

Simulation results for the approximate and the optimal controls are presented in Fig. 16 and Fig. 17 respectively. Notice that the residual enegry of the approximation is not zero. Depending on the mission requirement this resulting values may or may not be admissible. 
Table 4: Terminal Time for Two Flexible Modes

\begin{tabular}{l|l|l|l|l|l}
\hline$\omega_{2}$ & 1.036 & 1.294 & 2 & 4.741 & 6.672 \\
\hline$\omega_{1}$ & 1.1600 & 1.072 & 1.0053 & 1.0009 & 1.0000 \\
\hline$\frac{t_{f}}{t_{f_{1}}}$ & & & & & \\
\hline
\end{tabular}




\section{Some Extensions}

In previous chapters we have considered a simplified model for the rotating flexible structure. We have neglected both, structural damping and inherent nonlinearities associated with the slewing maneuvers. The problem has been further simplified by assuming ideal time response (zero lag) for the actuator. This assumptions cnabled a simple parameter optimization scheme to be applied, the solutions obtained by which have been shown to possess interesting symmetric and asymptotic properties.

In this chapter we aim to extend these results by considering the previously neglected effects of structural damping and nonlinear 'centrifugal' stiffness. The problem of realistic actuator with finite (nonzero) time lag is also studied where the time lag is taken as a parameter.

These extensions require two-point boundary-value problem solver for the numerical computations since an application of the simple parameter optimization, although feasible, is significantly complicated by the new formulation. 


\subsection{Damping}

For simplicity, we introduce constant damping coefficients $\xi_{j}, j=1,2, \ldots k$ in the modal space. The state space formulation of section 3.1 is still valid where the matrix $\mathrm{A}$ is changed to

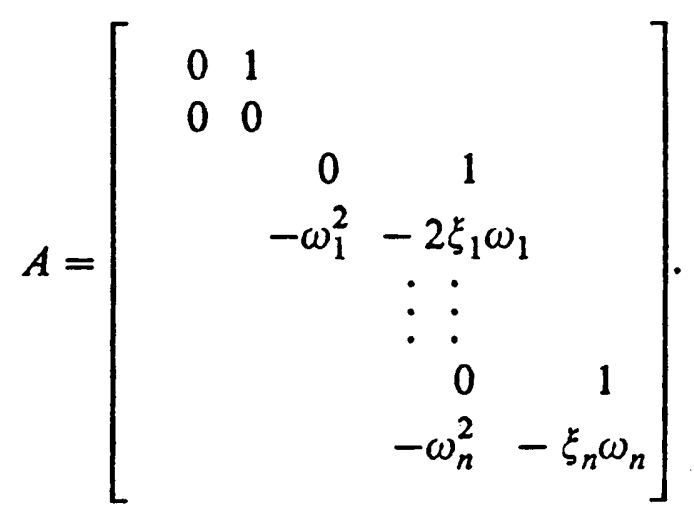

The time-optimal control problem can still be solved by the parameter optimization scheme (section 3.2) with appropriate step response functions. Another possible approach is to solve directly the two-point boundary-value problem with initial iteration data taken from the simpler, undamped solutions. We shall pursuc this latter approach here, since it also applies to the nonlinear problem and to the nonideal actuator case.

In order to transform the problem to fixed boundaries, as required by the numerical scheme, we intoduce a new independant variable

$$
\tau=\frac{t}{t_{f}}
$$

The terminal time satisfics 
$\frac{d t_{f}}{d \tau}=0$

Equation (3.3) is transformed to

$\frac{d \bar{x}}{d \tau}=(A \bar{x}+b u) t_{f}$

and equation (3.6) to

$\frac{d \lambda}{d \tau}=-A^{T} \lambda t_{f}$

The boundary conditions are now given at $\tau=0$ and at $\tau=1$.

Notice that (5.3)-(5.5) is a $(2 n+1)$ dimensional set of nonlinear differential equations ( $\mathrm{n}$ being the dimension of $\mathrm{A}$ ) resulting from the free terminal time of the originally linear problem.

Solutions for the problem have been obtained using BOUNDSOL, a computer code which employs a multiple shooting algorithm to solve two-point boundaryvalue problems [23]. Fig. 18 presents the optimal solution for the test-case $\left(x^{\circ}=30\right)$ with one flexible mode and where $\xi_{1}=0.01$. The number of switching points has not been changed by the damping effect, but the symmetry has been broken. The intermediate switching point is not at the midpoint, and the other two are not equally distant from it. The change in the transition time with respect to the undamped case is negligible. 


\subsection{Nonlinear Effects}

As already indicated in chapter 2 we shall retain for the analysis the nonlinearity term associated with the 'centrifugal' stiffness, neglecting the rest of the nonlinear term. The terms considered become significant in fast slewing maneuvers which are of interest in this work. The equations of motion are therefore (2.21)

$\ddot{\zeta}+\Omega^{2} \zeta=G U-\left(G^{i} \zeta\right)^{2} D \zeta$

Let

$T_{1}=\left[\begin{array}{lllllll}1 & 0 & 0 & 0 & 0 & 0 & . . \\ 0 & 0 & 1 & 0 & 0 & 0 & . \\ 0 & 0 & 0 & 0 & 1 & 0 & 0 \\ \cdots & \cdots & \cdots & \cdots & \cdots & \cdots\end{array}\right]$

$T_{2}=\left[\begin{array}{lllllll}0 & 1 & 0 & 0 & 0 & 0 & . . \\ 0 & 0 & 0 & 1 & 0 & 0 & \ldots \\ 0 & 0 & 0 & 0 & 0 & 1 & \ldots \\ \cdots & \cdots & \cdots & \cdots & \cdots\end{array}\right]$

where both matrices are $\left(\frac{n}{2} \times n\right)$. Using our former definition for $\bar{x}$, equation (3.2), we obtain

$\zeta=T_{1} \bar{x}$,

$\dot{\zeta}=T_{2} \bar{x}$.

The state space representation is therefore 
$\dot{\bar{x}}(t)=A \bar{x}(t)+b u(t)-T_{2}^{T}\left(G^{T} T_{2} \bar{x}(t)\right)^{2} T_{1} \bar{x}(t) \equiv f(\bar{x}(t) ; u(t))$

where $\mathrm{A}$ and $\mathrm{b}$ are given in equations (3.4) and (3.5). To solve the time-optimal problem (for rest-to-rest maneuvers) we define the Hamiltonian

$H(\bar{x}, u, \lambda)=1+\lambda^{T} f(\bar{x}, u)$.

By the Maximum Principle [20]

$u(t)=-u_{m} \operatorname{sgn}\left(\lambda^{T}(t) b\right)$

$\dot{\lambda}_{i}(t)=-\frac{\partial H(\bar{x}(t), u(t), \lambda(t))}{\partial x_{i}(t)} \quad i=1,2 \ldots . n$

and

$H\left(x\left(t_{f}\right), u\left(t_{f}\right), \lambda\left(t_{f}\right)\right)=0$

Thus, the solution is cither bang-bang or singular. It is noted that existence of a solution is guaranteed by the convexity of the hodegraph [20], but uniqueness and nonsingularity can not be guarantecd duc to the nonlinearity of the system.

Fig. 19 presents the solution for the test-case maneuver (obtained by BOUNDSOL) where one-flexible mode has been taken into account. Similarly to the linear case the solution is nonsingular, the number of switching points is three and their symmetry is prescrved. The solution is, in fact, a very small perturbation of the linear case. The problem was solved with two clastic modes and 
similar results have been obtained. In order to further investigate the nonlinear effects we increase the maximum admissible torque by the factor 10 , where $p=0,1, \ldots 6$. The angular velocities, and hence the nonlinear effects, should increase accordingly. Numerical results for the one-flexible-mode case indicate that the switching-point structure is preserved.

The distance between switching points $\bar{\Delta}$ is shown in Fig. 20 for both linear and nonlinear solutions. As expected, the linear solutions approach the smalltransition-time asymptotes ( $\omega$ is fixed). The nonlinear solution has a smaller asymptotic value. The transition time is presented in Fig. 21 on a logarithmic scale. The asymptotic straight line of the linear solution has a slope of -0.25 as predicted by (4.7). The solutions for the stiffer nonlinear system have, as expected, a smaller transition time. Fig. 22-23 present typical trajectories for the linear and nonlinear systems respectively. For this example $p=3$. Notice that the displacements for the nonlinear system are significantly smaller. By the nonlinear term, the switching-function curve $(\sigma)$ has been distorted from being a simple linear $+\sin$, but it is still nonsingular and it has the same number of symmetrically-located switching points.

\subsection{Symmetric Properties of Rest-to-Rest Maneuvers}

We have found numerically that for the undamped system, linear or nonlinear, the solutions for rest-to-rest maneuvers possess the symmetry 
$u(t)=-u\left(t_{f}-t\right)$

and that this property is not preserved if the system is damped. To prove this fact analytically we make two basic observations.

1. Rest-to-rest maneuvers have the property that if $u(t), t \in\left[0, t_{f}\right]$ is the minimum-time control which slcws the system by $\theta$ then $-\mathrm{u}(\mathrm{t}), t \in\left[0, t_{f}\right]$ is the minimum-time control which slews it by - $\theta$. This may be called spatial symmetry, and it holds for both the linear and the nonlinear model, with or without damping.

2. The equations of motion for a conservative system are invariant with respect to time direction. This may be called temporal symmetry and it holds for the undamped system only. (linearity has nothing to do with this property.)

In particular, equation (5.6) is invariant under a change of the independent variable from $t$ into $\bar{t}=t_{f}-t$. However, an introduction of a damping term $\Pi \dot{\zeta}$, where $\Pi$ is any constant matrix, breaks this temporal symmetry. In solving the time-optimal slewing, for a conservative system, one may use the temporal symmetry to reformulate the problem with the new independent variable $\bar{t}$. The symmetric propcrty (5.16) is now obtained from the spatial symmetry, since the same differential equations hold for moving forward or backward in time, but the rotational maneuver is reversed.

We note that this result is valid for other cost functionals as well. This is confirmed by the results presented by [3-5] for LQR problems. A proof for the time-optimal control problem of the linear system is also provided by [15]. The 
proof is by construction and it lacks the explanatory power of our approach. Moreover, it cannot be generalized to a nonlinear model or to other costs.

\subsection{Nonideal Actuator}

Consider the linear undamped system of equations (3.3). We assume first order actuator's dynamics

$\dot{u}(t)=\frac{1}{\tau}\left(-u(t)+u_{c}(t)\right)$

where $u_{c}$ is the commanded torque and $\tau$ is defined to be the actuator's time constant. We augment the state equations with the actuator equation to obtain

$\frac{d \hat{x}(t)}{d t}=\hat{A} \hat{x}(t)+\hat{b} u_{c}(t)$

where

$\hat{x}=\left[\begin{array}{l}u \\ x\end{array}\right]$

$\hat{A}=\left[\begin{array}{cc}\frac{-1}{\tau} & 0 \\ b & A\end{array}\right]$,

and 


$$
\hat{b}=\left[\begin{array}{l}
1 \\
\tau \\
0
\end{array}\right] \text {. }
$$

The time-optimal control problem is to find $u_{c}$ which drives

$$
\hat{x}_{0}=\left[\begin{array}{c}
0 \\
x_{0}
\end{array}\right]
$$

to a target set $\Theta$ in minimum time, subject to

$$
\left|u_{c}(t)\right| \leq u_{m}
$$

The system is normal [20] and the unique solution is bang-bang determined by

$$
\frac{d \hat{\lambda}(t)}{d t}=-\hat{A}^{T} \hat{\lambda}(t)
$$

$\tilde{u}_{c}(t)=-u_{m} \operatorname{sgn}\left(\hat{b}^{T} \hat{\lambda}(t)\right)=-u_{m} \operatorname{sgn} \hat{\lambda}_{1}(t)$

and $\hat{\lambda}\left(t_{f}\right)$ is orthogonal to $\Theta$. For rest-to-rest maneuvers with a free terminal torque this last condition yields $\hat{\lambda}_{1}\left(t_{f}\right)=0$.

Fig. 24-25 present trajectories for the test-case maneuver with $\tau=0.5 \mathrm{sec}$ and $\tau=0.1 \mathrm{sec}$, respectively. Notice that the number of switching points is still three, and that they are shifted backward in time (with respect to the ideal actuator case). The terminal time is increased by approximately $\tau$ seconds. Solving the problem for other values of $\tau$ we obtain switching points as shown in Fig. 26. The transition time is shown in Fig. 27 and the residual energy in Fig. 28. Based on 
this results one may design the system, trading off residual energy with transition time, similarly to the technique suggested by [10]. 


\section{Problem Analysis in Infinite Dimensional Space}

In previous chapters a spatially discrete model has been used to model the flexible structure of interest. In this chapter the time-optimal control slewing problem is analyzed in infinite dimensional space. We set up abstract evolution equations in a Hilbert space with an encrgy-like norm, and we demonstrate the wcllposedness of the model. It is shown that, under this formulation, the system is approximately controllable. The system, however, is not exactly controllable in the proposed Hilbert space. The time-optimal control problem is analyzed next, and a discussion about convergence properties of the approximated solutions is given. 


\subsection{Model Formulation}

The linearized governing equations are (section 2.5)

$m(\ddot{w}(x, t)+x \ddot{\alpha}(t))+\left(E I w^{\prime \prime}(x, t)\right)^{\prime \prime}=0$,

$I_{h} \ddot{\alpha}(t)-E I w^{\prime \prime}(0, t)=u(t)$,

with the boundary conditions

$w(0, t)=w^{\prime}(0, t)=w^{\prime \prime}(l, t)=w^{\prime \prime \prime}(l, t)=0$.

Recall that $\mathrm{EI}$ is the beam rigidity, $\mathrm{m}$ is the mass distribution, $l$ is the length of the beam, $I_{h}$ is the hub moment of inertia, $\alpha(t)$ is the angular orientation of the hub, $w(x, t)$ is the transverse deflection of the beam, and $\mathrm{u}(\mathrm{t})$ is the applied control torque. ( $)^{\prime}$ is a derivative with respect to $\mathrm{x}$, and () with respect to t. We follow $[24]$ in the state-space presentation.

Let

$z(t)=\left[\begin{array}{c}\dot{\alpha}(t) \\ w^{\prime \prime}(x, t) \\ \dot{w}(x, t)+x \dot{\alpha}(t)\end{array}\right]$

in the state space $Z=R \times L_{2} \times L_{2}$. Then, formally at least, the system (6.1)-(6.3) can be written as

$\dot{z}(t)=A z(t)+b u(t)$ 
The operator $\mathrm{A}$ is

$$
A=\left[\begin{array}{ccc}
0 & \frac{E I}{I_{h}} \delta_{0} & 0 \\
0 & 0 & D^{2} \\
0 & -\frac{E I}{m} D^{2} & 0
\end{array}\right],
$$

where $\delta_{0}$ denotes evaluation at $\mathrm{x}=0$, and $D$ denotes differeniation with respect to x. The domain of the operator $\mathrm{A}$ is given by $D(A)=\left\{\left(z_{1}, z_{2}, z_{3}\right) \in R \times H^{2} \times H^{2} \mid z_{2}(l)=0, z^{\prime}{ }_{2}(l)=0, z_{3}(0)=0, z^{\prime}{ }_{3}(0)=z_{1}\right\}$

where $H^{2}$ is the appropriate Sobolev space [25].

We define an inner product on $Z$ to be

$<z, y>=I_{h} z_{1} y_{1}+\int_{0}^{l} E I y_{2} z_{2} d x+\int_{0}^{l} m y_{3} z_{3} d x$

So that the square of the induced norm is twice the encrgy of the system.

The operator $b$ is bounded

$$
b=\left[\begin{array}{c}
I_{h}^{-1} \\
0 \\
0
\end{array}\right] .
$$


We shall prove that $\mathrm{A}$ generates a $C_{0}$ contraction semigroup. It can be shown that $\mathrm{A}$ is closed and that its domain is dense in $\mathrm{Z}$. We shall calculate $A^{\times}$, the adjoint oparator. By definition, if $0=\langle A y, \bar{w}>-<y, \tilde{w}>$ for every $y \in D(A)$, then $\tilde{w}=A^{\times}(\bar{w})$.

Thus,

$0=E I y_{2}(0) \bar{w}_{1}+E I \int_{0}^{l} y_{3}{ }^{\prime \prime} \bar{w}_{2} d x-E I \int_{0}^{l} y_{2}{ }^{\prime \prime} \bar{w}_{3} d x$

$$
-I_{h} y_{1} \tilde{w}_{1}-E I \int_{0}^{l} y_{2} \tilde{w}_{2} d x-m \int_{0}^{l} y_{3} \tilde{w}_{3} d x
$$

Integrating by parts, we obtain by the fundamental lemma of the Calculus of Variation

$$
\begin{aligned}
& \tilde{w}_{3}=\frac{E I}{m} \bar{w}_{2}^{\prime \prime}, \tilde{w}_{2}=-\bar{w}_{3}^{\prime \prime}, \tilde{w}_{1}=-\frac{E I}{I_{h}} \bar{w}_{2}(0) \\
& \bar{w}_{1}=\bar{w}^{\prime}{ }_{3}(0), \bar{w}_{2}(l)=\bar{w}_{2}^{\prime}(l)=\bar{w}_{3}(0)=0 .
\end{aligned}
$$

Therefore, A is skew-adoint $\left(A^{\times}=-A\right)$. By Phillips' theorem [26, $\left.\mathrm{p} 188\right]$ we conclude that $\mathbf{A}$ generates a norm-preserving group.

To include $\alpha$ in our state vector, we consider the space $W=R \times Z$, and augmenting the $\mathrm{z}$ vector by $\alpha$ 
$\bar{z}(t)=\left[\begin{array}{l}\alpha(t) \\ z(t)\end{array}\right]$

we obtain the state equation

$\dot{\bar{z}}(t)=\hat{A} \bar{z}(t)+\hat{b} u(t)$

Where

$\hat{A}=\left[\begin{array}{ll}0 & e_{1} \\ 0 & A\end{array}\right] ; e_{1}=\left[\begin{array}{lll}1 & 0 & 0\end{array}\right]$

with the domain

$D(\hat{A})=R \times D(A)$.

Here $\hat{b}$ is given by

$\hat{b}=\left[\begin{array}{l}0 \\ b\end{array}\right]$

We define the inner product

$<\bar{z}, \bar{y}>=\bar{z}_{1} \bar{y}_{1}+I_{h} \bar{z}_{2} \bar{y}_{2}+\int_{0}^{l} E I \bar{y}_{3} \bar{z}_{3} d x+\int_{0}^{l} m \bar{y}_{4} \bar{z}_{4}$.

$\hat{A}$ is composed of an infinitesimal generator of a $C_{0}$ semigroup 


$$
\left[\begin{array}{ll}
0 & 0 \\
0 & A
\end{array}\right]
$$

plus a bounded operator

$$
\left[\begin{array}{ll}
0 & e_{1} \\
0 & 0
\end{array}\right]
$$

Therefore, by standard theorems $\left[27\right.$, p. 76], it generates a $C_{0}$ semigroup $\mathrm{S}$ on W (no longer norm-preserving).

\subsection{Exact and Approximate Controllability}

A system is said to be exactly controllable if it can be driven in a finite time from any given point to every point in the state space. Since the operator $b$ is compact, and the state space is infinite-dimensional, it can be concluded that the system is not exactly controllable in $Z$ (or W) [26, p.233].

If the set of all reachable points is dense in the state space, then the system is approximately controllable. We shall show that the system (6.5) is approximately controllable.

A sufficient condition for approximate controllability is given in [26, p.235]. Suppose that for some $T>0$

$b^{\times} e^{A^{\times}} z=0$ for $0 \leq t \leq T$ 
implies $z=0$, then the system is approximately controllable.

For the system (6.5)

$e^{A^{\times} t}=e^{-A t}$

$b^{\times}=[1,0,0]$.

Substituting (6.19) into (6.18), we obtain

$[1,0,0] e^{-A t} z=\dot{\alpha}(-t)=0 \quad 0 \leq t \leq T$.

which implies

$\ddot{\alpha}(-t)=0 \quad 0 \leq t \leq T$.

The homogeneous solution to equation (6.2) satisfics

$w^{\prime \prime}(0,-t)=0 \quad 0 \leq t \leq T$.

Hence, the homogencous solution to equations (6.1)-(6.3) is

$w(x,-t)=0 \quad 0 \leq t \leq T$.

Thus, $z$ in (6.18) is necessarly the zero vector and the system is controllable. We have not obtained a similar proof for the system (6.13) in the space W. However, finite-dimensional approximations indicate that controllabilty is preserved for the extended system (see section 2.3). 


\subsection{Time Optimal Control}

Consider now the time-optimal slewing maneuver to a given point in $\mathrm{W}$. We assume the existence of a control function that accomplishes this task in a finite time. The existence of timc-optimal control (and an optimal time $T$ ) is then guaranteed [26].

We can apply now the bang-bang principle [see Appendix] ( $\hat{b}$ is constant and bounded) to claim that for every $\varepsilon>0$ there exists a bang-bang control (the number of switchings is finite) that drives the system in the time $T$ to an $\varepsilon$ neighborhood of the target.

We want now to further characterize the optimal control, thus we are looking for

a certain form of the Maximum Principle. Since $\hat{b}$ is compact, the attainable set is compact and, therefore (being also closed), it cannot contain an interior point (since a compact set in a Hilbert space of infinite dimension can not have an interior point [26]). In this case a boundary point is not necessarly a support point and, therefore, the Maximum Principle is not directly applicable [26]. It is, howcver, possible to apply the Maximum Principle (as in fact we have done in previous chapters) to finite dimensional subspaces of $\mathrm{W}$, to obtain an approximated sequence of (bang-bang) controls. Notice that by assuming the expansion (2.10)

$w(x, t)=\sum_{i=1}^{k} \eta_{i}(t) \Psi_{i}(x)$, 
we are considering a projection to subspace $W_{n}^{\prime}$ of $\mathrm{W}$ spanned by $\mathrm{n}=2 \mathrm{k}+2$ basis vectors [19]

$$
\left[\begin{array}{l}
1 \\
0 \\
0 \\
0
\end{array}\right] ;\left[\begin{array}{l}
0 \\
1 \\
0 \\
x
\end{array}\right] ;\left[\begin{array}{c}
0 \\
0 \\
\Psi_{i^{\prime \prime}}(x) \\
0
\end{array}\right] ;\left[\begin{array}{c}
0 \\
0 \\
0 \\
\Psi_{i}(x)
\end{array}\right]
$$

where $\mathrm{i}=1,2, \ldots \mathrm{k}$.

The state space equations in this subspace are

$\dot{\bar{z}}_{n}(t)=\hat{A}_{n} \bar{z}_{n}(t)+\hat{b}_{n} u(t)$

where $\bar{z}_{n}=P_{n} \bar{z}$ (the projection of $\mathrm{z}$ to $W_{n}$ ), $\hat{A}_{n}$ is an operator in $W_{n}$ which generates a semigroup $S_{n}$, and $\hat{b}_{n}$ is a bounded operator from $R$ to $W_{n}$. The machinery of the assumed-mode method has been used in chapter 2 to construct representations in $R^{n}$ for $\hat{A}_{n}$ and $\hat{b}_{n}$.

We assume that for every $\bar{z} \in W$.

$S_{n}(t) P_{n} \bar{z} \rightarrow S(t) \bar{z}$

and that

$\hat{b}_{n} \rightarrow \hat{b}$

Consider rest-to-rest slewing mancuvers. Let $\tilde{u}_{n}$ be the time-optimal control which drives $P_{n} z_{0}$ to the origin of $W_{n}$ in an optimal time $T_{n}$. Assume 
$T_{n} \rightarrow T$

To justify this assumption we note that by increasing the order of the approximation we increase the number of the controlled modes, and we also increase the flexibility of the structure. Thus, from previous work, we anticipate a monotone increase of $T_{n}$, and therefore a convergence from below to $\mathrm{T}$.

$\tilde{u}_{n}$ is a bang-bang control governed by a switching function $\sigma_{n}$ [26]

$\sigma_{n}(t)=\hat{b}_{n}^{\times} S_{n}^{\times}\left(T_{n}-t\right) \lambda_{n}$

where $\lambda_{n} \in W_{n},\left|\lambda_{n}\right|=1$. For our system we can rewrite the last equation

$\sigma_{n}(t)=<\hat{b}_{n}, S_{n}^{\times}\left(T_{n}-t\right) \lambda_{n}>=<S_{n}\left(T_{n}-t\right) \hat{b}_{n}, \lambda_{n}>$

A subsequence of $\lambda_{n}$ converges weakly to $\lambda_{0}$ (because $\left|\lambda_{n}\right|=1$ ), renumbering it we obtain

$\lambda_{n} \rightarrow \lambda_{0} \quad$ weakly.

Since

$S_{n}\left(T_{n}-t\right) \hat{b}_{n} \rightarrow S(T-t) \hat{b} \quad$ strongly,

we conclude that

$\sigma_{n}(t) \rightarrow<S(T-t) \hat{b}, \lambda_{0}>$ 
Thus, unless $\lambda_{0}=0$, the solutions converge to a bang-bang optimal control in $L_{2}(0, T)$. Notice that the case $\lambda_{0}=0$ cainnot be cxcluded a priori in our formulation. It is noted that recently Fattorini [see Appendix] discussed the convergence problem in a more general setting for the time-optimal control problem.

Fig. 29-30 present the switching functions for the two cases studied in chapter 3 , for one, two and three controlled modes $(k=0, k=1$ and $k=2)$. The controls seem to converge in $L_{2}(0, T)$ by the fact that new switching points are either perturbations of the old ones, or else they apear in couples, creating narrow pulses with a negligible effect on the integral from 0 to $\mathrm{T}$. 


\section{Time Optimal Control with Penalty on the}

\section{Control Effort}

In this chapter we consider a different approach for fast maneuvers of flexible spacecraft. As opposed to the pure minimum-time problem, the cost here is a weighted combination of the terminal time and a measure of the control effort (soft-constrained time optimization). The problem is solved for the discrete system by solving the associated two-point boundary-value problem. The increasing value of the natural freqencies in modal space is a key point in the development of a singular-perturbation technique which decomposes the modal space into reduced and residual subspaces. The basic approach is to fix the terminal time by the reduced model and to provide a correction to the reduced model control by the solution of a residual problem. 


\subsection{Problem Formulation}

We consider the linear state space model (3.3) (omitting the bar from $\mathbf{x}$ )

$\dot{x}(t)=A x(t)+b u(t)$

where

$A=\left[\begin{array}{ccccc}0 & 1 & & & \\ 0 & 0 & & & \\ & & 0 & 1 & \\ & & -\omega_{1}^{2} & -2 \xi_{1} \omega_{1} & \\ & & & \vdots & \\ & & & 0 & 1 \\ & & & -\omega_{k}^{2} & -2 \xi_{k} \omega_{k}\end{array}\right]$

$b=\left[\begin{array}{llllllllll}0 & g_{0} & 0 & g_{1} & \ldots & . & 0 & g_{k}\end{array}\right]^{T}$.

The optimal control problem is to find a measurable function $u$ which drives the state $x$ from a given value $x(0)$ to a target point $x\left(t_{f}\right)$ while minimizing

$J=t_{f}+\frac{1}{2} \int_{0}^{t_{f}} \rho u(t)^{2} d t \quad ; \quad \rho>0$

i.e. a weighted combination of the terminal time and a measure of the control effort. 


\subsection{Optimality Conditions}

The system is linear, stable, and controllable and the cost is strictly convex. Existence and uniqueness of the optimal solution arc, therefore, guaranteed, and the Maximum Principle is a sufficient as well as necessary condition for it [20]. Let $\tilde{u}$ be the optimal control, we define the Hamiltonian

$H(x, \lambda, u)=1+\frac{1}{2} \rho u^{2}+\lambda^{T}(A x+b u)$.

We obtain by the Maximum Principle

$\dot{\lambda}(t)=-A^{T} \lambda(t)$,

$\tilde{u}(t)=-\rho^{-1}\left(\lambda^{T}(t) b\right)$,

and

$H(x(t), \lambda(t), \tilde{u}(t))=0$.

The optimal control can be constructed by solving a two-point boundary-value problem. Normalizing the system by the unknown terminal time, we obtain (the independent variable is the normalized time $\tau=t / t_{f}$ )

$\frac{d t_{f}}{d \tau}=0$ 
$\frac{d x}{d \tau}=(A x+b u) t_{f}$

$\frac{d \lambda}{d \tau}=-A^{T} \lambda t_{f}$

and the boundary conditions are given at $\tau=0$ and at $\tau=1$.

\subsection{Computational Results}

The two-point boundary-value-problem was solved by a Multiple-Shooting Algorithm (MSA) [23]. The system was discretized by the first three assumed modes of (2.10) and the damping ratio for all modes was 0.02 . The rest-to-rest slewing problem was investigated, where the system is to be driven from

$x=[-15,0, \ldots .0]^{T}$,

to the origin, while minimizing the cost $\mathrm{J}$ with $\rho=10^{-4}$ (chosen to produce reasonable controls and costs).

Three cases were considered. A rigid body problem where only the zcroth natural frequency is taken into account (case a), a rigid body plus the fundamental frequency problem (case b), and a system with two elastic modes (case $\mathrm{c}$ ). The time histories of the optimal solutions are presented in Figures 31-33, where the terminal time is $2.0,2.46$ and 2.45 seconds respectively. As might be expected the 
terminal time converges as we increase the dimension of the truncated model. In fact, we could fix the value of the terminal time as obtained by case $b$, and then solve case $\mathrm{c}$ as a fixed duration problem, minimizing the control effort, to obtain a good approximation for the higher dimensional case (Fig. 34).

For the linearized system, the problem, after fixing the time, is linear and thus easy to solve. For the nonlinear model or for problems where a higher dimensional model is necessary in order to obtain the convergence in the terminal time, it is tempting to try to construct approximate solutions by a perturbation from the reduced order, already obtained solution, and by so doing, to reduce the computational effort. This reduction provides, therefore, the motivation for a singular perturbation approach.

\subsection{Solutions by a Singular Perturbation Technique}

We partition the state equations

$$
\left[\begin{array}{l}
\dot{x}_{o} \\
\dot{x}_{r}
\end{array}\right]=\left[\begin{array}{cc}
A_{o} & 0 \\
0 & A_{r}
\end{array}\right]\left[\begin{array}{l}
x_{o} \\
x_{r}
\end{array}\right]+\left[\begin{array}{l}
b_{o} \\
b_{r}
\end{array}\right] u .
$$

The optimal control problem is to drive the system from

$$
x=\left[\begin{array}{c}
x^{0} \\
0
\end{array}\right]
$$

to the origin in a given time $t_{p}$ while minimizing 
$\bar{J}(u)=\frac{1}{2} \int_{0}^{t_{f}} u(t)^{2} d t$

The adjoint set of equations is

$\left[\begin{array}{l}\dot{\lambda}_{o} \\ \dot{\lambda}_{r}\end{array}\right]=-\left[\begin{array}{ll}A_{o}^{T} & 0 \\ 0 & A_{r}^{T}\end{array}\right]\left[\begin{array}{l}\lambda_{o} \\ \lambda_{r}\end{array}\right]$,

and, by the Maximum Principle

$\tilde{u}=-b_{o} \lambda_{o}-b_{r} \lambda_{r} \equiv \tilde{u}_{o}+\tilde{u}_{r}$

Based on the increasing value of the natural frequencies we make the following assumptions:

1. $\left|x_{o}\left(t ; \tilde{u}_{r}\right)\right|<<\left|x_{o}\left(t ; \tilde{u}_{o}\right)\right|$

2. $x_{r}\left(t ; \tilde{u}_{o}\right) \sim-A_{r}^{-1} b_{r} \tilde{u}_{o}(t)$.

Where $x(t ; u)$ is the response at time $t$ due to the control $u$ (with zero initial condition). The idea behind the first assumption is that the equations of the lower natural frequencies behave like low-pass filters to the high frequency content of the control function. The second assumption assumes a zero time lag in the response of the high frequency cquations to the slowly varying part of the control. These two assumptions form the basis for the singular perturbation algorithm. 
A reduced system is formed by neglecting the high frequency content of the control (in accordance with the first assumption). We obtain the reduced state equations

$\dot{x}_{o}=A_{o} x_{o}+b_{o} u_{o}$,

and the boundary conditions

$x(0)=x^{0} ; \quad x\left(t_{f}\right)=0$

Minimizing $\bar{J}\left(u_{o}\right)$ we obtain the reduce order optimal control $\hat{u}_{o}$. The residual state equations are

$\dot{x}_{r}=A_{r} x_{r}+b_{r} u_{r}$.

However, the target point is now (in accordance with the second assumption)

$x_{r}\left(t_{f}\right)=A_{r}^{-1} b_{r} \hat{u}_{o}\left(t_{f}\right)$

(For the initial condition, we may or may not assume that the steady-state response has been obtained. It turns out that it has a negligible effect on the optimal-control time history for this problem.)

Let $\hat{u}_{r}$ be the optimal solution minimizing $\bar{J}\left(u_{r}\right)$. The optimal solution of the original problem is thus approximated by the composite

$\hat{u}=\hat{u}_{0}+\hat{u}_{r}$ 
We may use the problem of the previous section as a numerical example. Fig. 35 compares the exact solution of case $\mathrm{c}$ with a composite solution obtained from case $b$ and a residual correction. The approximate control for this example is in excellent agreement with the exact optimal solution.

As indicated earlier, the computationally more important cases are problems with nonlinear models or problems where the reduced model is necessarly of high dimension (e.g. closely spaced natural frequencies). The system under investigation belongs to the former class of problems. Fig. 36 and Fig. 37 present time histories for a linear and nonlinear models of the system respectivly. Two flexible modes were taken into account. The final time is fixed and is equal to 1 second. Notice that the linear is more flexible than the nonlinear case.

Fig. 38 compares the exact nonlinear solution with an approximate solution obtained by singular perturbation. The reduced model is, again, the one flexible mode case and the residual model is the equation of the second elastic mode. The approximate results are in good agrcement with the results for the unreduced model of two flexible natural frequencies. 


\section{Conclusions and Recommendations}

\subsection{Summary and Conclusions}

In the course of this work, the problem of single-axis time-optimal slewing for a flexible structure has been studied using several mathematical models with different degrecs of complexity. The first model was a truncated lincarized model. The problem has been transformed to a constrained parameter optimization problem, and a verification method has been developed to remove the inherent uncertainty of the parameter optimization. This approach can be applied to other problems where the solution is known to be bang-bang and it is not restricted to our specific application.

With the motivation to synthesize a control law for onboard computers, certain approximations have been suggested based on asymptotic properties of the solutions. In these approximations the control strategy is given in a very-simple closed form. 
Based on the lincar model, the nonlinear problem has been solved by a Multiple Shooting Algorithm. Some interesting symmetric properties of the switching structure are shown to be common to both models. The MSA has been further used to remove two other idealizations, the assumption of no-damping and the assumption of ideal actuators. By removing the latter assumption, a family of solutions has been constructed to demonstrate the effect of the actuators's time lag on the time-optimal solutions.

The problem has been studied in Hilbert space with energy-like norm. After showing well-posedness the controllabilty issue has been addressed. It has been shown that the system is approximately but not exactly controllable in the proposed space. Approximate solutions for the minimum-time problem are shown to converge through the convergence of the switching function.

The last problem addressed in this work, is a minimization of time and control effort (soft-constrained time-optimal control problem). Being simpler than the hard-constrained problem, a singular-perturbation technique can be applied to it, to reduce the computational effort. This idea can be applied to a broader class of problems.

We note that the hard-constrained and the soft-constrained problems are not entirely independent. The optimal control of the latter, equation (7.7), has the same form as the switching function of the former, hence a certain correlation exists between the two. For example, the number of switches in the one-flexible mode case is the same as the number of zero crossing of the soft-constrained problem control function (Fig. 32). For small transition times (Fig. 36) these 
zero-crossing points approach the asymptotic value of the time-optimal switches. Indeed, the solutions of the soft-constrained problem as $\rho \rightarrow 0$ approach the time-optimal solutions as $u_{m} \rightarrow \infty$.

\subsection{Comparison with Some Other Works}

We have already indicated that the time-optimal slewing problem has been studied by others in the past two years. Since only preliminary results have been reported, any comparison will be necessarily partial. We shall discuss briefly the works of Rajan [13], of Baker and Polak [14], and of Singh, Kabamba and McClamrock [15].

In [13] the problem is studied in a linearized truncated space. The target sct, however, is an $\varepsilon$ ball in this space rather than a single point. This relaxes the problem to some extent and affects the solutions. The computational approach is to directly solve the two-point boundary-value problem, which, in spite of the analytical integerability of subarcs, is still a computational burden. To the extent that the set-target solutions can be indicative to point-target solutions, a comparison of the results is sufficiently good. In particular, the observation made about the perturbation-like character of the switches is in agreement with our analytical and numerical results. 
The approach in [14] is to consider the problem with the nonlinear term in infinite dimensional form, relaxing the target to be an $\varepsilon$ ball around the target point. The algorithm is conceptually a gradient method, and it requires discretization in space (FEM) and in time. The computational effort is significant. The computational results are in qualitative agreement with ours. The switching structure obtained is consistent with the one-flexible mode case of our formulation.

The approach in [15] is more similar to ours. The problem is considered in a truncated linearized modal space, and the target is a point. The discussion is restricted to rest-to-rest maneuvers. In stead of the parameter optimization technique described above, the problem in [15] is transformed into solving a set of nonlinear equations by homotopy methods. The algorithm actually uses the symmetric properties of the switches and a proof is provided to establish this fact. As already indicated, the proof in [15] is restricted to the linear time-optimal control problem. In general, there is an agreement between the results of [15] and the results obtained in chapter 3 of this work (sec also [16]). The two approaches seem to be, more or less, equivalent in the computational effort. Our approach is, however, less specific and can be applied to other mancuvers and to other problems with or without structural elements. The nonlinear system and the realactuator case are not considered in [15]. 


\subsection{Recommendations}

It is important to emphasize that open-loop control, based on just a few modes, is probably inadequate for systems with many, closely-spaced, natural frequencies, especially if the knowledge of the system is not complete. This, unfortunately, is often the case for large flexible structure. For these systems, closed-loop control, based on actual measurements, is necessary. A practical approach in space missions (for time-optimal maneuvers) might be a combination of our open-loop approach with the closed-loop approach of $[11,12]$. Thus, it is suggested to control, in an open-loop fashion, the first few elastic modes, and in closed loop, perhaps by a different control device, the higher modes. The forcing term, taken by [11,12] in the elastic equations, is now less destructive, and the control effort will be diminished accordingly. We believe that even controlling the fundamental mode (perhaps via asymptotic properties) might be beneficial. This, however, is left for further study to determine.

Another idea for further study is to include structural parameters in the timeoptimization process. Thus, we can imagine designing a system, with certain functional and structural constraints, to meet the target of minimum time. The parameter optimization technique of chapter 3 is complctely adequate for this process and some interesting results are expected.

Beyond the scope of the particular problem of time-optimal slcwing we believe that the ideas of chapter 3 (parameter optimization technique with optimality 
verfication) and chapter 8 (singular perturbation based on natural frequencies) can be applicable to other applications in future rescarch. 


\section{Appendix : A Brief History of the Minimum-Time}

\section{Problem}

\section{Introduction}

The history of time-optimal problems is also, to some extent, the history of optimal control theory since this class of problems marked the way to most important developments in this field. Hermes and LaSalle [22] indicated that there is no other problem, in control theory, about which our knowledge is as complete as in case of the finite-dimensional time-optimal control problem. Most of this knowledge was developed during the years 1949-1960, in two important centers, the RAND corporation in the US and the Academy of Sciences of the USSR. An important contribution, namely the bang-bang principle, is also credited to LaSalle himself. The timc-optimal control problem in infinite-dimensional space 
has been investigated ever since 1960 and this process has not been completed yet.

We shall try to sketch here the history of the problem, examining the main ideas and concepts rather than going into mathematical details. Therefore, we shall confine the discussion to contributions of a more general and basic nature rather than to specific problems and applications of time-optimal controls.

\section{The Calculus of Variations Approach}

The first minimum-time problem, known as the brachistochrone problem, was proposed and discussed by the Bernoulli brothers in the seventeenth century. A bead decends by gravity along a frictionless wire, and the problem is to find the shape of the wire for a minimum time of decent. Problems in Calculus of Variations of a similar kind have been continuously considered ever since, and numerous ideas and techniques have been developed to deal with them.

During the Second World War a German aircraft designer A. Lippisch [28] applied the methods of the Calculus of Variations to control problems of atmospheric flight. Unfortunately, he did not get the right formulation of EulerLagrange equations for his problem. In 1949, M. Hestenes at the RAND corporation considered a minimum-time problem in connection with aircraft climb performance [29]. He applied the methods of the Calculus of Variations, considering it as the problem of Bolza by means of a device used by Valentine. He was among the first to formulate the Maximun Principle as a translation of 
the Weierstrass condition. Unfortunatly, the original work [29] was never published and these results were not available for a considerable time. Berkovitz [30] presented Hestenes' work and indicated that it is more general than the Maximum Principle, since the case of state-dependent control bounds is also included, where the Maximum Principle considers only controls that lie in a fixed closed set.

\section{Phase Plane Approachs}

The first work to consider the time-optimal control problem, outside the framework of the calculus of variation, was Bushaw's Ph.D. dissertation in 1952, under the guidance of Professor S. Lefschetz, at Princeton University [31,32].

He corisidered the following nonlinear oscillator

$\ddot{x}+g(x, \dot{x})=\phi(x, \dot{x})$,

where $\phi(x, y)$ assumes only the values -1 and 1 . We are to find $\phi$ that drives in minimum time the state $(x, \dot{x})$ from $\left(x_{0}, y_{0}\right)$ to the origin. This formulation was motivated by the intuition that using the maximum available power yields the best results for the minimum-time problem.

The approach was to study possible trajectories in phase plane. It was shown that only 'canonical paths' are candidates for optimal trajectories. A 'canonical path' does not contain a switch from -1 to 1 in the upper half plane $(\dot{x}>0)$ or a switch from +1 to -1 in the lower half plane $(\dot{x}<0)$. Solutions were obtained for the linear case, i.e. where $\mathrm{g}$ is a linear function, with complex eigenvalues. 
As indicated by LaSalle [22], this approach could not be generalized to problems with more degrees of freedom. One idea however, in this work has been generalized. Bushaw asserted that: If $\Delta$ is the solution of (1) starting at point $p$, and $\mathrm{p}^{\prime}$ is any point on $\Delta$, then the solution curve starting at $\mathrm{p}^{\prime}$ is that part of $\Delta$ which follows $\mathrm{p}^{\prime}$. A direct generalization to this is the principle of optimality.

In the same year, LaSalle [33] showed that for equation (1), given $g$, if there is a unique 'bang-bang' system which is the best of all 'bang-bang' systems, then it is the best of all possible systems operated from the same power source . Thus, it is the optimal solution for every $\phi$ that satisfies $|\phi(x, y)| \leq 1$. This seems to be the first time that the terminology 'bang-bang' was applied to time-optimal problems.

At the same period of time, a similar phase plane approach was applied to time-optimal problems in the Soviet Union by Fel'dbaum [34]. In particular, solutions were obtained for the double integrator system

$\ddot{x}=u$

$|u(t)| \leq M$

and the problem of driving the system from point to point in minimum time. 


\section{The Maximum Principle}

A general approach for the time-optimal problem was developed by Bellman, Glicksberg and Gross [35] in 1954 at RAND Corporation. They considered the linear differential equation:

$\dot{x}(t)=A x(t)+f(t)$

where $\mathrm{A}$ is a (nxn) constant matrix with stable eigenvalues, and $\mathrm{f}$ is a $\mathrm{n}$ dimensional vector of measurable functions $f_{i}$ and $\left|f_{i}(t)\right| \leq 1$.

It was shown that there exist $f$ that drives the system to the origin in minimum time, and that for this $\mathrm{f}\left|f_{1}(t)\right|=1$. In addition to that, for real distinct eigenvalues, the number of switchings was shown to be no more than $(\mathrm{n}-1)$.

The approach applied to obtain these results is even more important than the results. They investigated the properties of what is called today the set of attainability, and showed that it is convex, that it is closed and that it varies continuously with time. It then follows that there exits a unit vector (normal to a supporting hyperplane) that satisfies a certain inequality which later has been named the maximum principle.

The n-dimensionality of the control space in Bellman's work is, of course, a very serious restriction, and as it has been shown later an unnecessary one.

The Maximum Principle itself has been developed at the same time in the Mathematics Institute of the Academy of Sciences of the USSR by a group of scientists under the leadership of Academician L.S. Pontryagin. The original 
proof for the Maximum Principle was based on the properties of the cone of attainability [36] obtained by variations of the control function. Application of the maximum principle to the linear time-optimal control problem yields a switching structure similar to [35].

Gamkrelidze [37] and Krasovskii [38] also considered the more general linear system

$\dot{x}(t)=A x(t)+B u(t)$,

where $\mathrm{A}$ is as above, $\mathrm{B}$ is a (nxm) matrix and the region for the controller $\mathrm{u}$ is $\mathrm{a}$ polyhedron in $R^{m}$. It was shown that if a certain condition called 'the general position condition' is satisfied then there exists a unique solution to the timeoptimal control problem. This condition has been renamed by LaSalle who called it 'the normality condition'.

In spite of the similarity in the approach and the results between the RAND group and Pontryagin's group, they seem to be independent developments [39], like some other great achievements in the history of science.

\section{The Bang-Bang Principle}

In the late 50's LaSalle developed his 'bang-bang' principle for linear systems [40]. He showed that for the system (4) any point that can be reached in a given time by an admissible control, can also be reached, at the same time, by a bangbang control, i.e. where $u(t)$ is, almost always, on a vertex of the given 
polyhedron. Applying to the time-optimal control problem, this means that if there is an optimal control then there exist also a 'bang-bang' optimal control. Therefore, if the optimal control is unique, e.g. if the system is normal (in the sense of the normality condition), then the unique solution is bang-bang.

LaSalle considered also a wider class of systems of the form (4) namely 'proper systems', that turned out to be completely controllable systems. He showed that for these systems optimal controls are, almost always, on the boundary of the polyhedron. Every normal system is proper, but a system can be proper without being normal. If, however, the system is proper and the control is a scalar, then the system is also normal.

Based on the observation made about the adjoint vector as a unit normal to the attainable set, a geometric interpetation for the maximum principle was offered by LaSalle. The optimal control selects the direction which maximizes its component in the direction of the outward normal to the attainable set. Strict convexity of the attainable set means a unique optimal solution, but does not mean a unique adjoint vector because of possible corners in the boundary.

In 1969, Hermes and LaSalle summarized the knowledge about the time-optimal problem for systems of ordinary differential equation in their monograph Functional Analysis and Time optimal Control [22]. A complete discussion is given for existence and uniqueness of solutions for linear systems as well as a brief discussion of the nonlinear time-optimal control problem. 


\section{Infinite Dimensional Space}

L. V. Egorov [41] was among the first to generalize the Maximum Principle to a certain class of equations in Banach space. He was followed by H. O. Fattorini [42] who considered a general problem in Hilbert space which is a natural extension of Bellman's problem [35] to infinite-dimensional space. Let

$\dot{x}(t)=A x(t)+f(t)$,

where $\mathrm{x}(\mathrm{t})$ and $\mathrm{f}(\mathrm{t})$ are in Hilbert space $\mathrm{H},|f(t)| \leq 1$, and $\mathrm{A}$ is an infinitesimal generator of a strongly continuous semigroup. It was shown, by the weakly compactness property, that if there is a control that drives $z_{1}$ to $z_{2}$, then there is also a time-optimal control that does it. Morcover, this control is unique and it cannot contain sub-arcs with $|f(t)|<1$.

A. Fricdman [43] extended these results to Banach Space, and obtained further results concerning the uniqueness problem in Hilbert space. He showed that if $A$ in (5) is a generator of a strongly continuous group $S$, and if there is a timeoptimal control where $T$ is the minimum time, then there is $p \neq 0$ in the state space such that the time-optimal control satisfies

$\tilde{u}(t)=\frac{S^{\times}(T-t) p}{\left|S^{\times}(T-t) p\right|}$

where $S^{\times}$is the adjoint of $\mathrm{S}$. The optimal control is therefore bang-bang just like the finite-dimensional case. If $\mathrm{A}$ is only assumed to generate a strongly contin- 
uous semigroup, then the attainable set may not have an interior point, and we can only asserts the existence of a weakly convergent sequence of bang-bang controls. This result had been previously proved by Balakrishnan [44] for the more general case (a natural extension to [37])

$\dot{x}(t)=A x(t)+B u(t)$

where $u$ and $x$ are not in the same Hilbert space and where $B$ is a bounded linear operator, and $|u(t)| \leq 1$. In particular, he proved that if the semigroup is compact, or if B is a compact operator, then there is no interior point to the attainable set and the optimal control is a weak limit of a bang-bang sequence.

Fattorini [45] also generalized the bang-bang principle to infinite dimenional space and asserted that a response to (7) can always be approximated by a bang-bang control.

G. Knowles [46] continued in the 70's to investigate the conditions for the solution to be bang-bang, a situation which he called 'normal' as a natural extension of LaSalle's original terminology.

Since most infinite-dimensional systems do not possess exact (point to point) controllability, but only approximate controllability (a dense set is always attainable), Knowles [47] and Ahmed [48] discussed a time-optimal problem with a target set $B\left(z_{2}, \varepsilon\right)=\left\{z:\left|z-z_{2}\right| \leq \varepsilon\right\}$, rather then exact hitting. Existence and uniquness of the solution were discussed along the same lines of the previous formulations. 
A problem of recent interest is the convergence of approximated (typically, finite-dimensional) time-optimal solutions to the optimal soltuion of the original system. Carja [49] and Fattorini [50,51] discussed the propertics of suboptimal controls of this kind and gave sufficient conditions for convergence. 


\section{References}

1. Turner, J.D., Optimal Large Angle Spacecraft Rotational Maneuvers, Ph.D. Dissertation, Virginia Polytechnic Inst. and State University, May, 1980.

2. Turner, J. D., and Junkins, J. L., Optimal Large-Angle Single-Axis Rotational Maneuvers of Flexible Spacecraft, J. of Guidance and Control, Vol. 3., No. 6, Nov.-Dec., 1980, pp. 578-585.

3. Turner, J. D., and Chun, H. M., Optimal Distributed Control of a Flexible Spacecraft During a Large Angle Maneuver, J. of Guidance and Control, Vol. 7., No. 3, May-June, 1984, pp. 257-264.

4. Juang, J., Turner, J. D., and Chun, H. M., Closed-Form Solution for Feedback Control with Terminal Constraints, J. of Guidance and Control, Vol. 8., No. 1, Jan.-Feb., 1984, pp. 39-43. 
5. Breakwell, J. A., Optimal Feedback Slewing of Flexible Spacecraft, J. of Guidance and Control, Vol. 4., No. 5, Sept.-Oct., 1981, pp. 472-479.

6. Lisowski, R. J., and Hale A.L., Optimal Design for Single Axis Rotational Maneuvers of a Flexible Structure, Journal of Astronautical Sciences, Vol. 32, April-June, 1985, pp. 197-206.

7. Juang, J., Horta, L. G., and Robertshaw, H. H., A Slewing Experiment for Flexible Structure, J. of Guidance and Control, Vol. 9, Sept.-Oct., 1986, pp. 599-607.

8. Skaar, S. B., Tang, L., and Yalda-Mooshabad, Y., On-Off Attitude Control of Flexible Satellites, J. of Guidance and Control, Vol. 9., July-Aug., 1986, pp. 507-510.

9. Vander Velde, W.E., and He, J., Design of Space Structure Control System Using On-Off Thruster, Journal of Guidance, Control, and Dynamics, Vol 6, No. 1, Jan.-Feb., 1983.

10. Thompson, R.C., Junkins J.L., and Vadali S.R., Near Minimum Time Open Loop Control of Dynamic Systems, AIAA Dynamics Specialists Conference, April, 1987. 
11. Meirovitch, L., and Sharony, Y., Optimal Maneuvcring of Flexible Spacecraft, Sixth VPI\&SU/AIAA Symposium on Dynamics and Control of Large Structures, June, 1987.

12. Quinn, R.D. and Meirovitch, L., Maneuvering and Vibration Control of Flexible Spacecraft, Workshop on Structural Dynamics and Control Interaction of Flexible Structures, NASA, Marshall Space Flight Center, Huntsville, Alabama, April, 1986.

13. Rajan N., Minimum-Time Slewing of the SIRTF Spacecraft, Proceedings of the AIAA Guidance, Navigation and Control Conference, Aug., 1987, pp. $1222-1227$.

14. Baker, T. E., and Polak E., Computational Experiments in the Optimal Slewing of Flexible Structure, Electronics Research Laboratories Memo. No. UCB/ERL M87/72, Univ. of California, Berkeley, Sept., 1987.

15. Singh, G., Kabamda P. T. , and McClamroch N. H., Time Optimal Slewing of a Rigid Body With Flexible Appendages, Proceedings of the 28th Conference on Decision and Control, Los-Angeles, CA, Dec., 1987. pp. 1441-1442.

16. Ben-Asher, J. Z., Burns J. A., and Cliff, E. M., Time Optimal Slewing of Flexible Spacecraft, Proceedings of the 28th Conference on Decision and Control, Los-Angeles, CA, Dec., 1987. 
17. Meirovitch, L., Computational Methods in Structural Dynamics, , Sijthoff \& Noordhoff Co., The Netheriands, 1980.

18. Hughes, P. C. and Skelton, R. E., Controllabilty and Observability of Linear Matrix-Second-Order Systems, Journal of Applied Mechanics, Vol. 47, June, 1980, pp. 415-420.

19. Burns, J. A., and Cliff E. M., Optimal Control of a Distributed System, Lecture Notes in Control and Informatiom Theory Series \#95, pp. 304-318, Springer-Verlag, Berlin, 1987.

20. Lee E.B, and Markus, L., Foundation of Optimal Control Theory, Krieger Publishing Company, Malabar, Florida, 1986.

21. Kelley, H.J.,and Speyer, J.L., Accelerated Gradient Projection, Lecture Notes on Mathematics 132, Springer-Verlag, Berlin, 1970.

22. Hermes, H., and Lasalle, J. P., Functional Analysis and Time Optimal Control, Academic Press, New York and London, 1969.

23. Bulirsch, R., Einfuchrung in die Flungbahnoptimierung ; Mehrzielmethode zur Numerischen Loesung von nichtlinearen Randwertproblemen und Aufgaben der Optimalen Steuerung, Lehrgang Flugbahnoptimierung CarlCranz-Gesellschaft e.v. ,October, 1971. 
24. Burns, J. A. et al., State Space Model and Advanced Control Concepts for Large space Structures, USAFRPL Report, Optimization Inc., Blacksburg, VA, April, 1985.

25. Adams, R. A., Sobolev Spaces, Academic Press, New-york, 1975.

26. Balakrishnan, A. V., Applied Functional Analysis, Springer-Verlag, NewYork, 1981.

27. Pazi, A., Semigroups of Linear Operators and Applications to Partial Differential Equations, Springer-Verlag, New-York, 1983.

28. Lippisch, A., Performance Theory of Airplane with Jet Propulsion, Translation Report No. F-TS-685-RE, Headquarters Air Material Command, Wright Field, Dayton, Ohio, 1946.

29. Hestenes, M. R., A General Problem in the Calculus of Variations With Applications to Paths of Least Time, The Rand Corporation, RM-100, March, 1950.

30. Berkovitz, L. D., Variational Methods in Problems of Control and Programming, Journal of Mathematical Analysis and Applications, Vol. 3, 1961, pp. 145-169. 
31. Bushaw, D. W., Ph.D. Thesis, Department of Mathematics, Princton University, 1952.

32. Bushaw, D. W., Optimal Discontinuous Forcing Term, Contributions to the Theory of Nonlinear Oscillations, Vol. 4, Princeton University Press, Princeton, N.J., 1958, pp. 29-52.

33. LaSalle, J. P., Basic Principle of the Bang-Bang Servo, Abstract 247t, Bulletin of the American Mathematical Society, Vol. 60, March, 1954, p. 154.

34. Fel'dbaum, A. A., Optimal Processes in Automatic Control Systems, Avtomatika i Telemekhanika, Vol. 14, No. 6, June, 1953, pp. 712-728.

35. Bellman, R., Glicksberg, I., and Gross O., On the Bang-Bang Control Problem, Quaterly of Applied Mathematics, Vol. 3, No. 1, April, 1956, pp. 11-18.

36. Pontryagin, L. S., Boltyanskii, V. G., Gamkrelidze, R. V., and Mishckenko, E. F., The Mathematical Theory of Optimal Processes, Wiley Interscience, New York, 1967.

37. Gamkrelidze, R. V., Thcory of Time-Optimal Processes for Linear Systems, Izvest. Akad. Nauk. SSSR 22, 1958, pp. 449-474. 
38. Krasovskii, N. N., Concerning the Theory of Optimal Control, Avtomatika i Telemekhanika, Vol 18, No. 12, Nov., 1957, pp. 960-970.

39. LaSalle, J. P., The Time Optimal Control Problem, Contributions to the Theory of Nonlinear Oscillations, Vol. 5, Princeton University Press, Princeton, N.J., 1959, pp. 1-24.

40. LaSalle, J. P., The Bang-Bang Principle, Proceedings of the First International Congress of the International Federation of Automatic Control, Moscow, 1960.

41. Egorov, L. V., Optimal Control in Banach Space, Dolk. Akad. Nauk. SSSR 150. 1963, pp. 241-244. (translated in Soviet Mathematics 4, 1963)

42. Fattorini, H. O., Time-Optimal Control of Solutions of Opcrational Differentinal Equations, SIAM J. Control, Vol. 2, No. 1, 1964, pp. 54-59.

43. Friedman, A., Optimal Control in Banach Spaces, Journal of Mathematical Analysis and Applications, Vol. 19, 1967, pp. 35-55.

44. Balakrishnan, A. V., Optimal Control Problems in Banach Spaces, SIAM J. Control, Vol. 3, No. 1, 1965, pp. 152-179. 
45. Fattorini, H. O., A Remark on the Bang-Bang Principle for Linear System in Infinite-Dimensional Space, SIAM J. Control, Vol. 6, No. 1, 1968, pp. 109-113.

46. Knowles, G., Time Optimal Control in Infinite Dimensional Systems, SIAM J. Control, Vol. 14, 1976, pp. 919-933.

47. Knowles, G., An Introduction to Applied Optimal Control, Academic Press, New York, 1981.

48. Ahmed, N. U., On the Maximum Principle for Time-Optimal Controls for a Class of Distributed-Boundary Control Problems, Journal of Optimization Theory and Applications, Vol. 45, No. 1, January, 1985.

49. Carja, O., On Variational Perturbations of Control Problems: MinimumTime Problem and Minimum-Effort Problem, Journal of Optimization Theory and Applications, Vol. 44, No. 3, November, 1984.

50. Fattorini, H. O., Optimal Control of Nonlinear Systems: Convergence of Suboptimal Controls I, Proceedings of Special Session on Operator Problems in Optimal Control Problems, Annual AMS Meeting, New Orleans, January, 1986. 
51. Fattorini, H. O., Optimal Control of Nonlinear Systems: Convergence of Suboptimal Controls II , Proceedings of IFIP Workshop on Control Systems Described by Partial Differential Equations, Gainesville , February, 1986. 


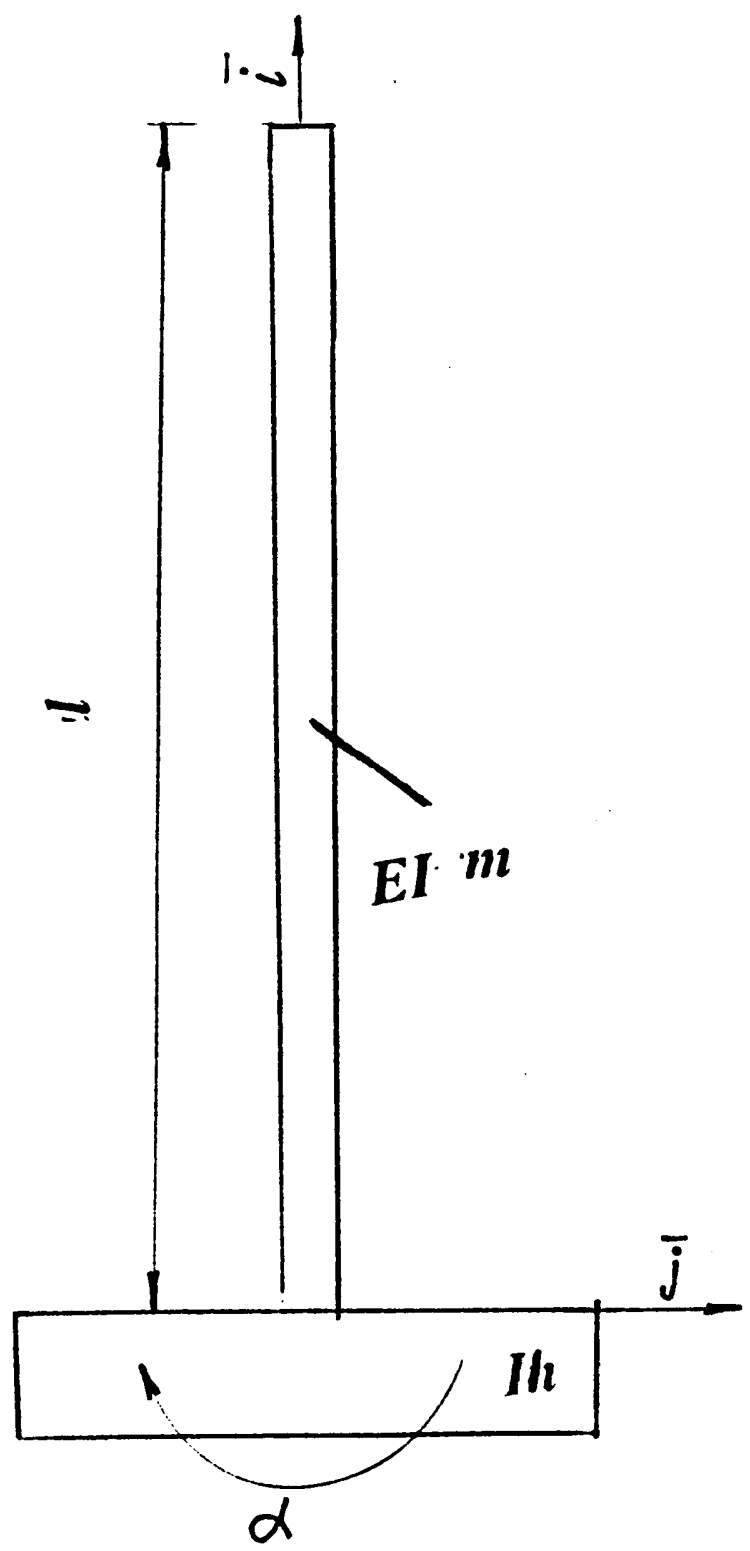

Fig. 1: Flexible Structure 


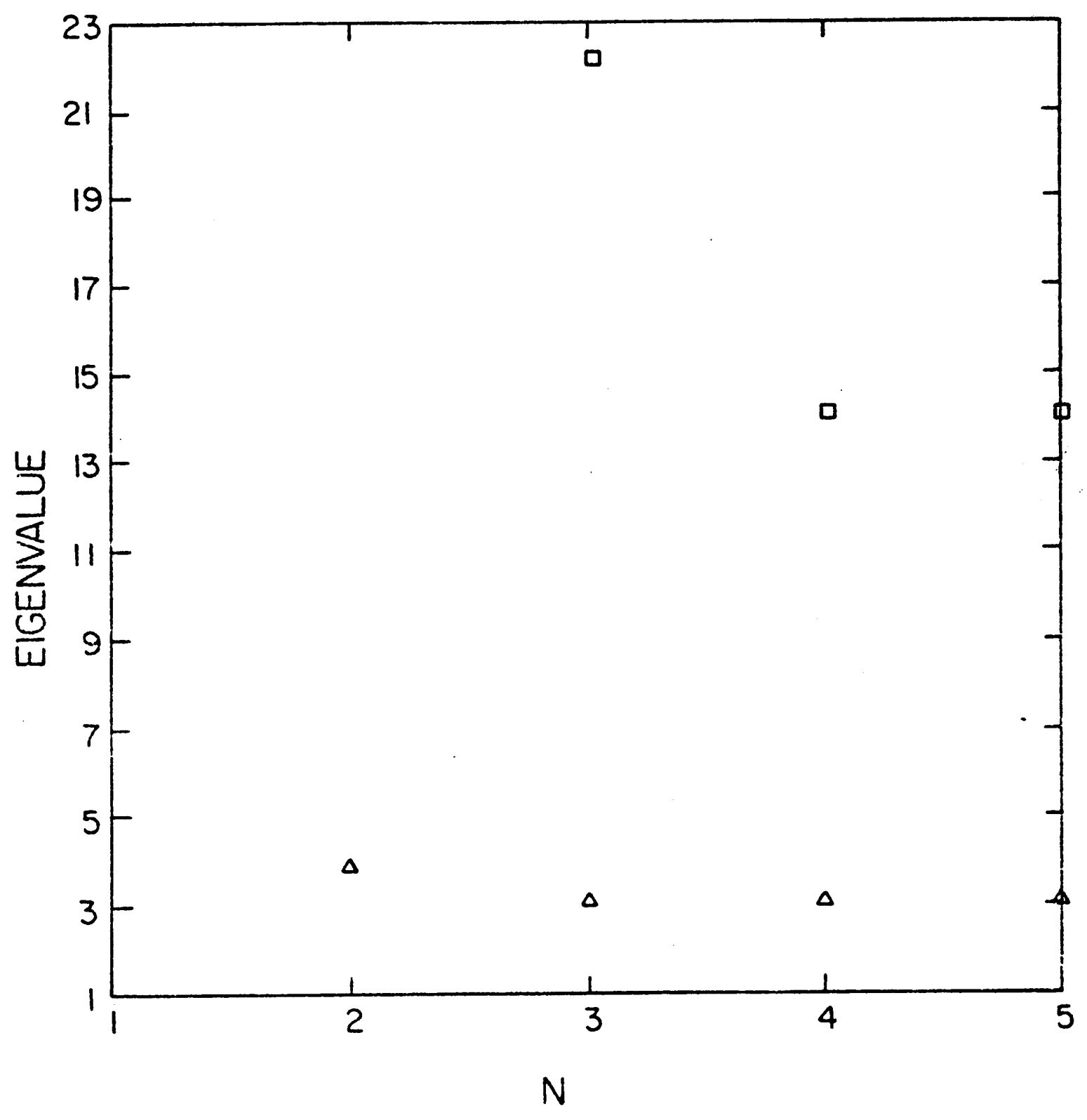

Fig. 2 : Natural Frequencies 


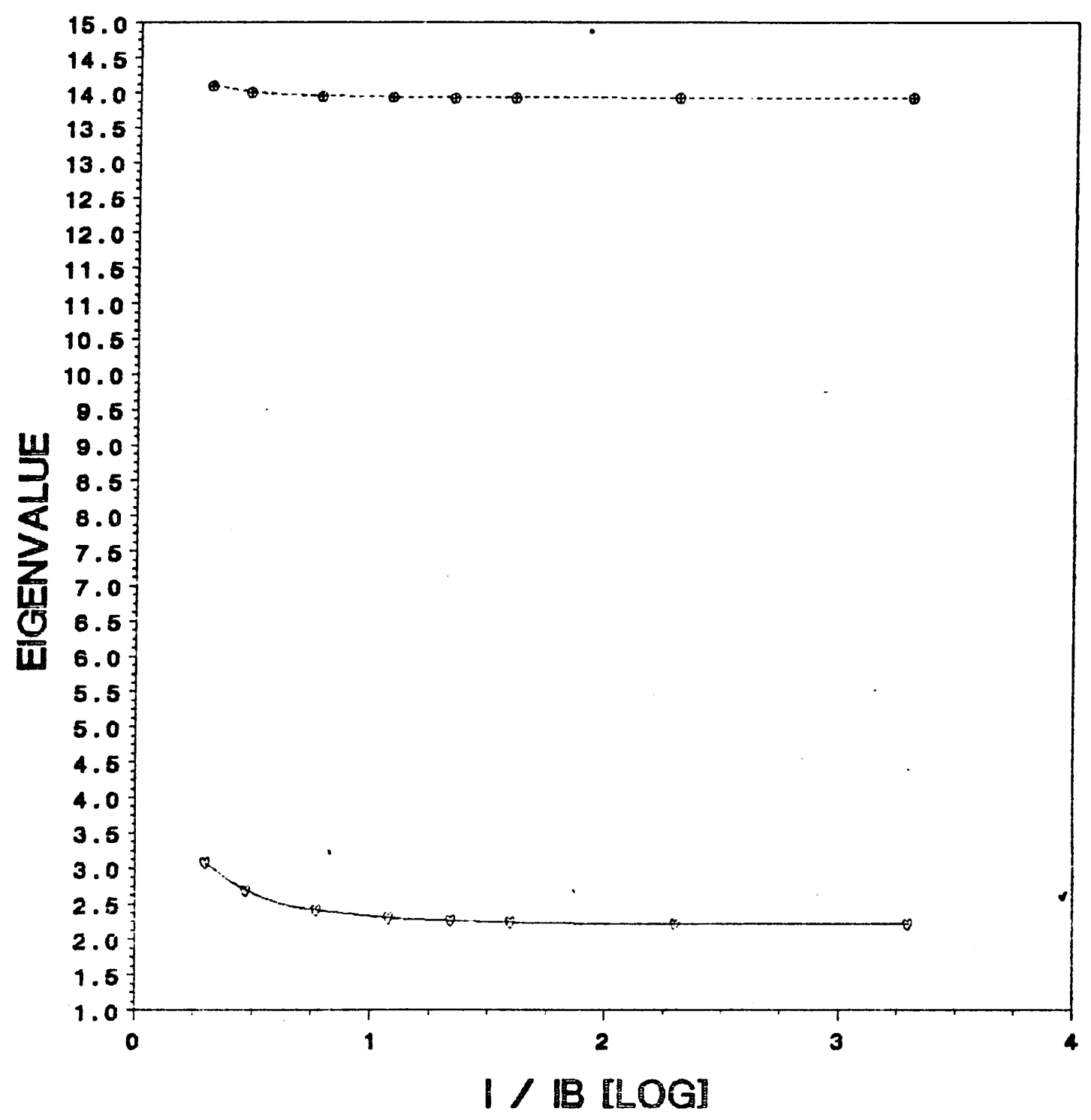

Fig. 3 : Natural Frequencies 

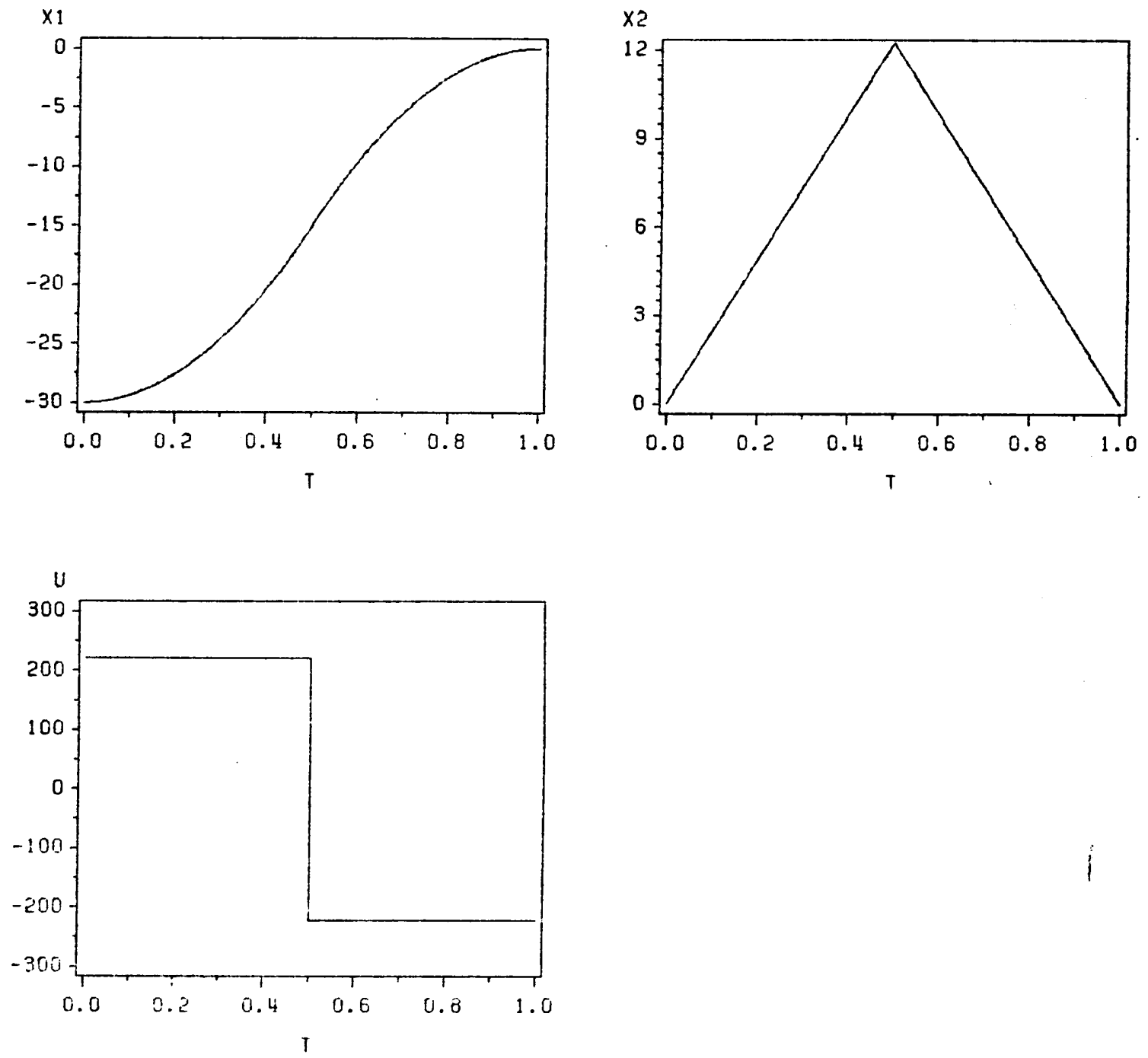

Fig. 4 : Rigid Body - Rest-to-Rest $\left(x^{o}=30\right)$ 

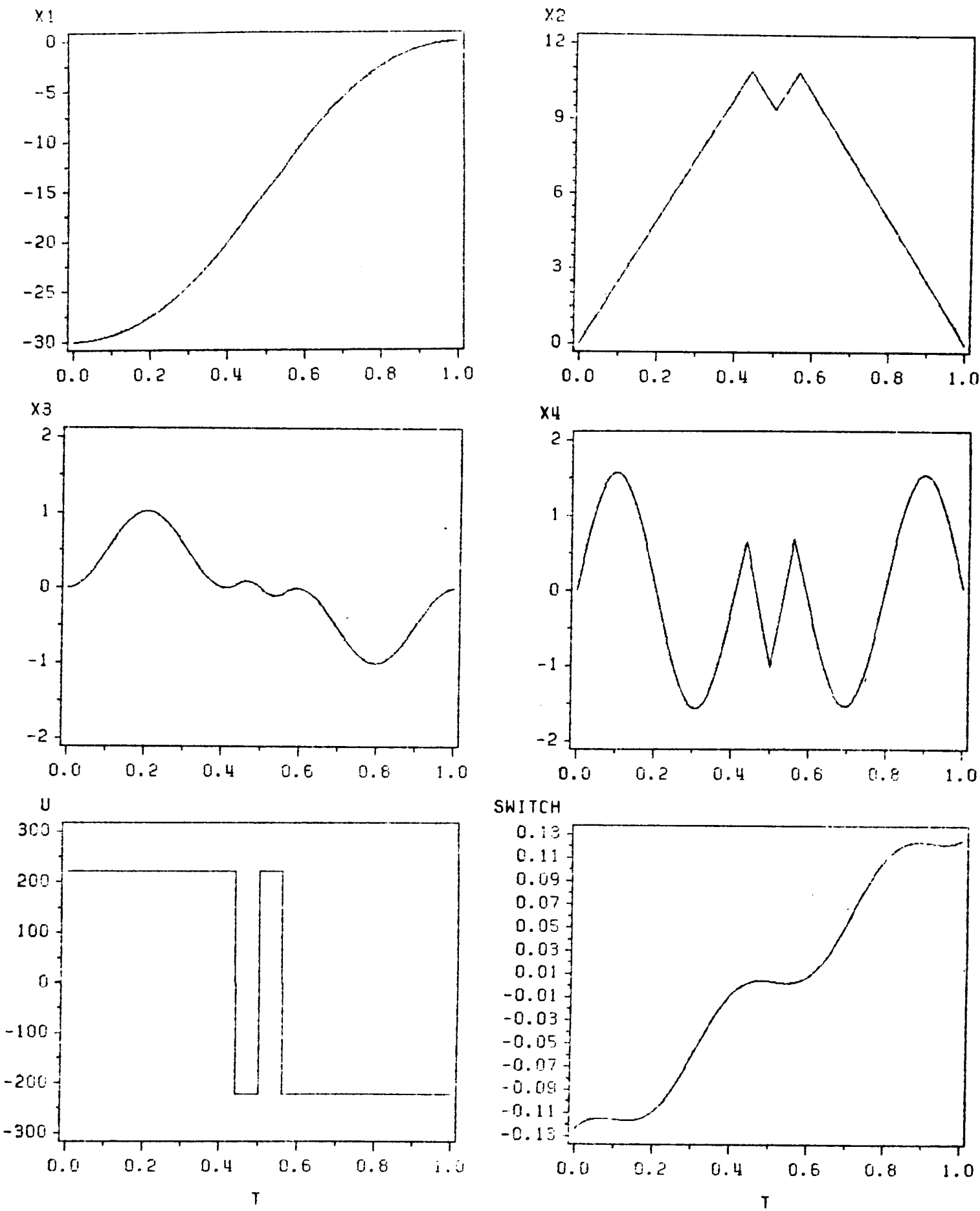

Fig. 5: One Elastic Mode - Rest-to-Rest $\left(x^{o}=30\right)$ 


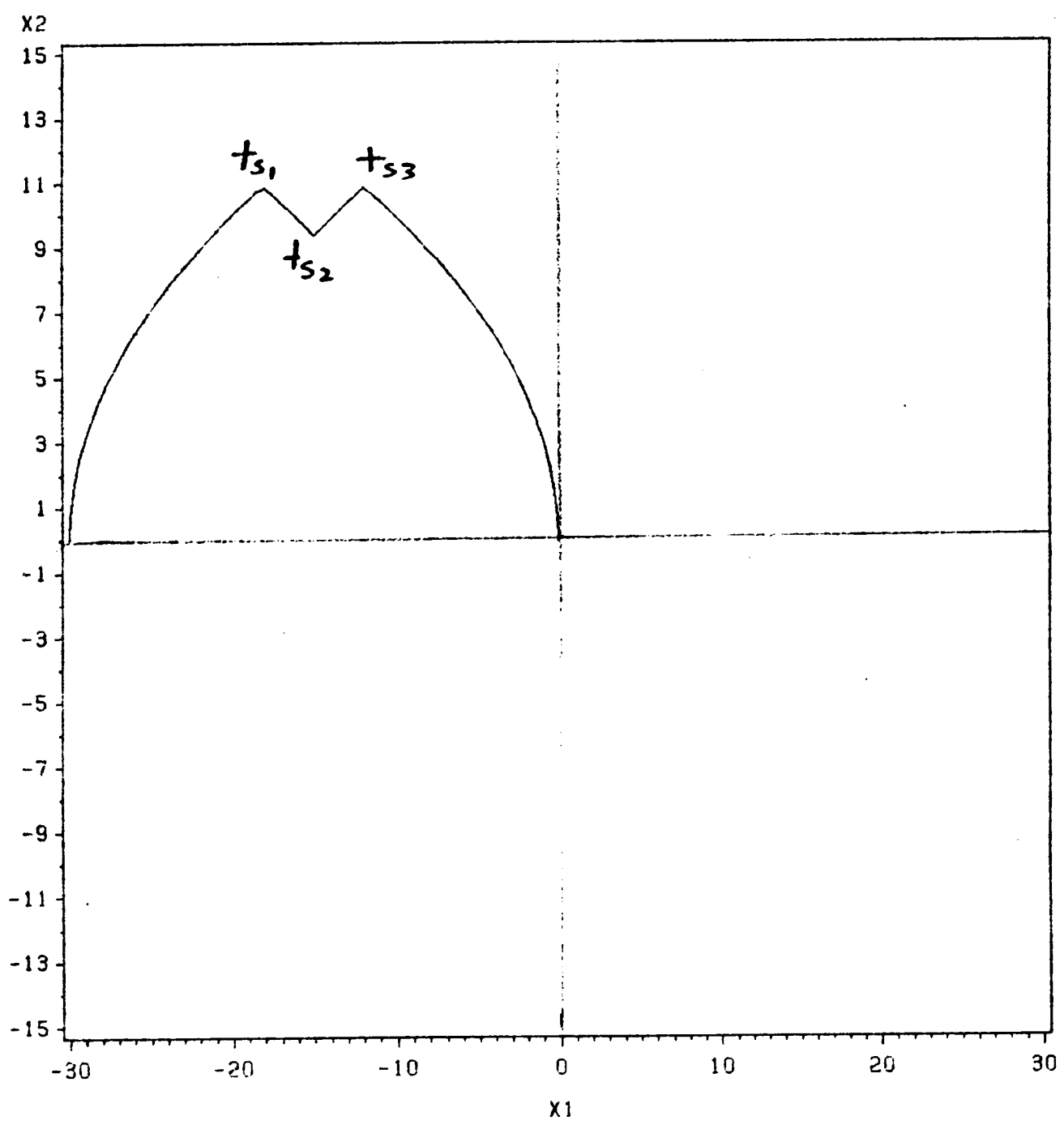

Fig. 6 : Phase Space-Rest-to-Rest 


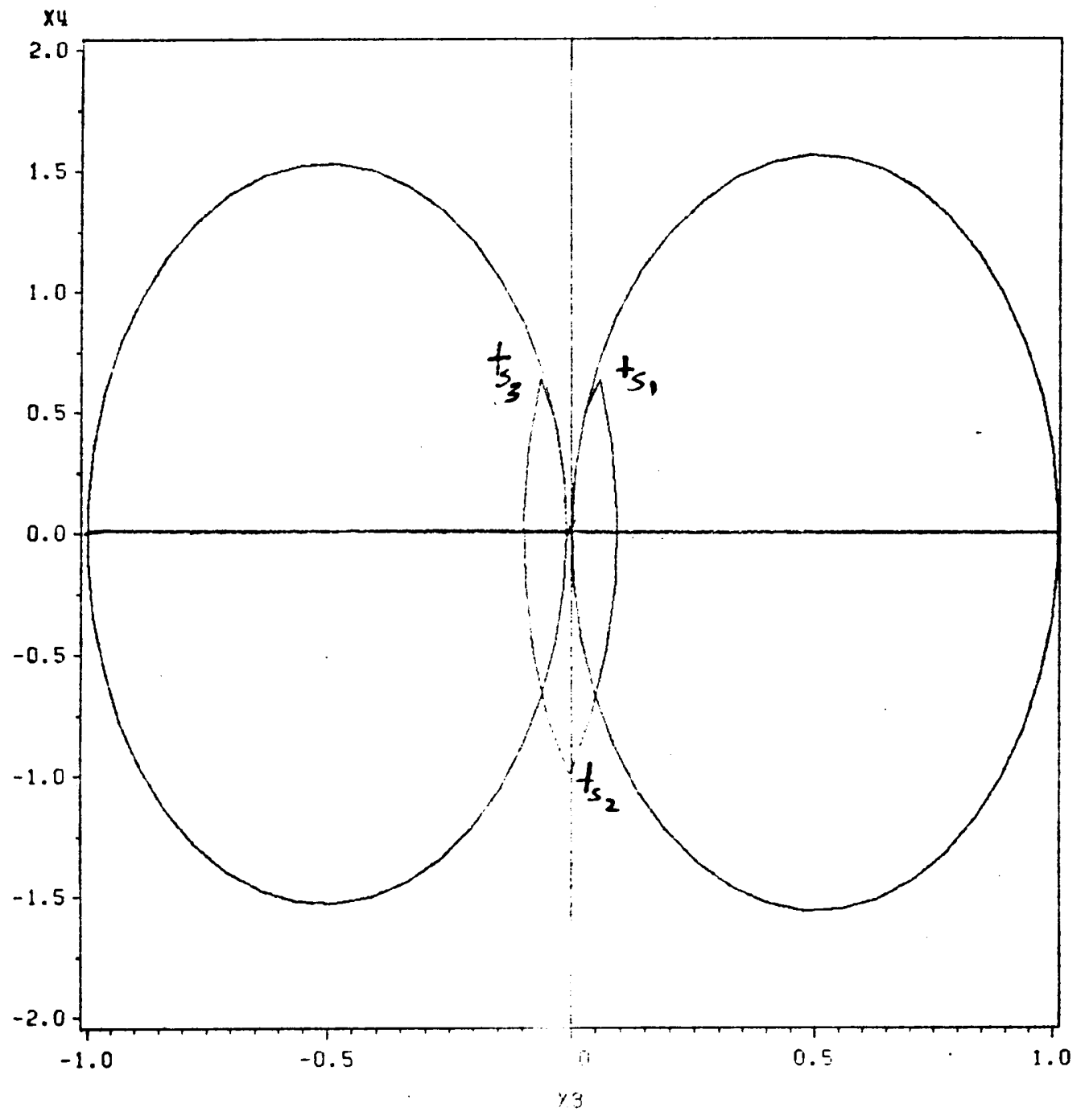

Fig. 7 : Phase Space - Rest-to-Rest 


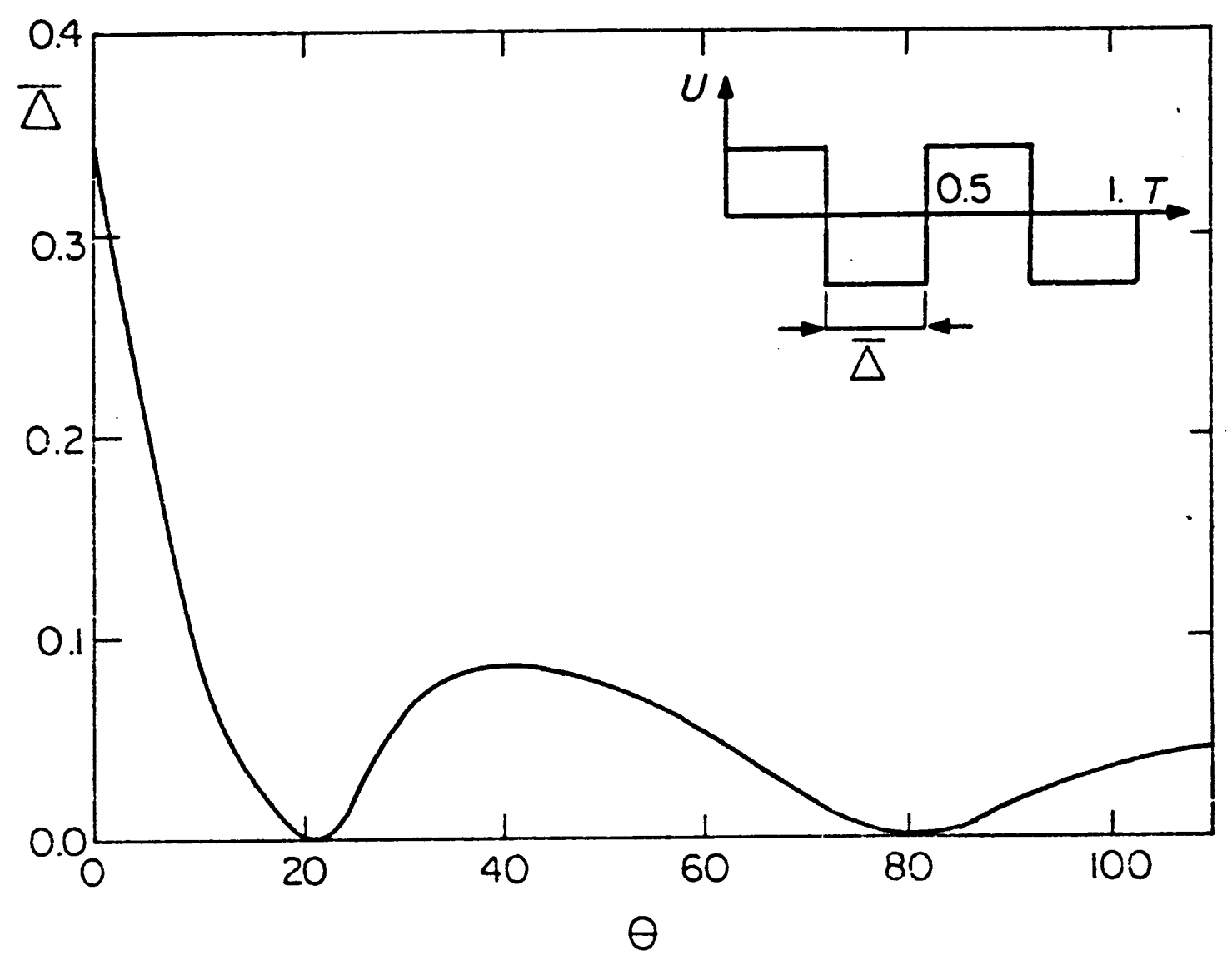

Fig. 8 : Switching Points vs. Slew Angle 


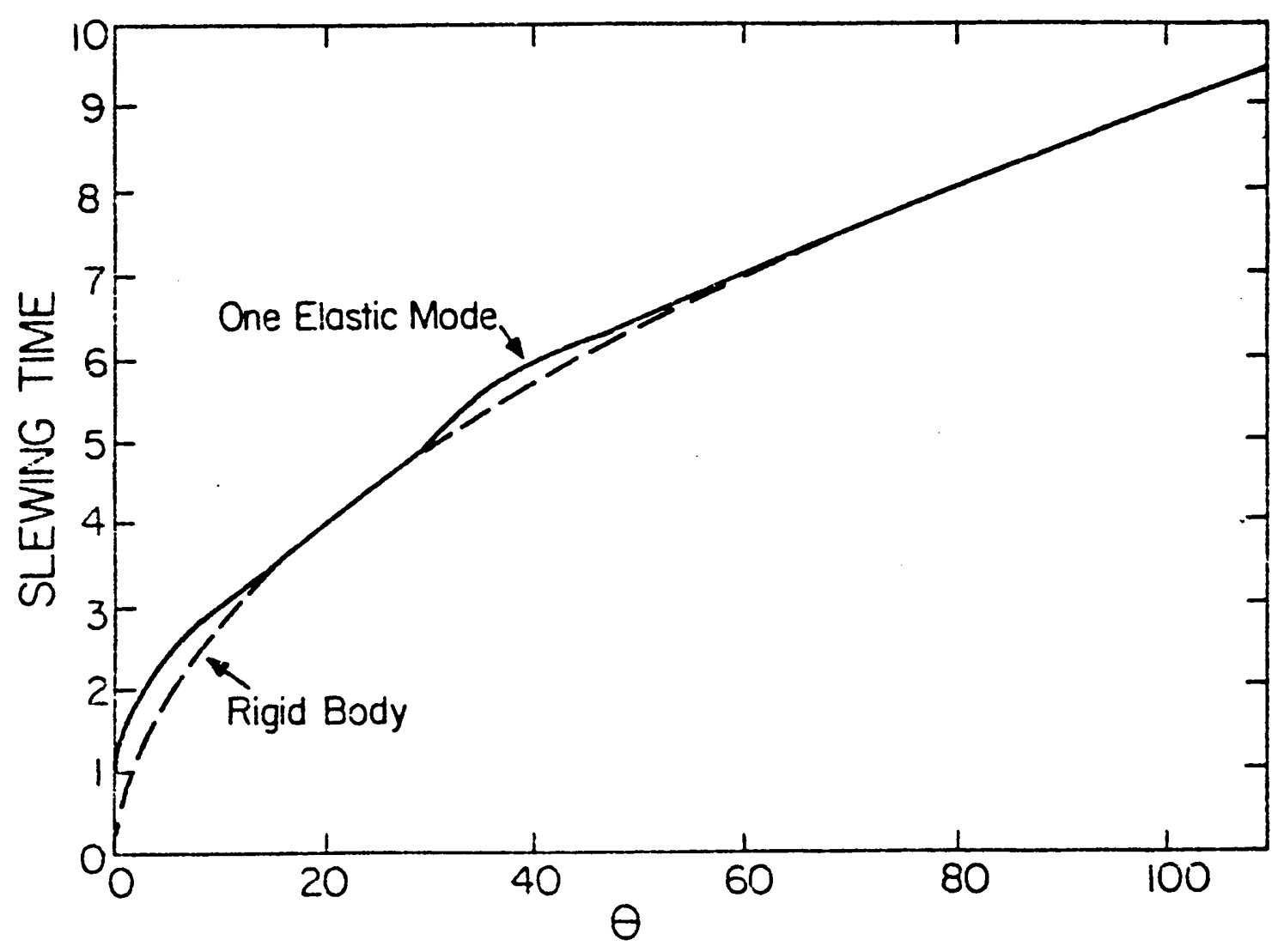

Fig. 9 : Slewing Time vs. Slew Angle 

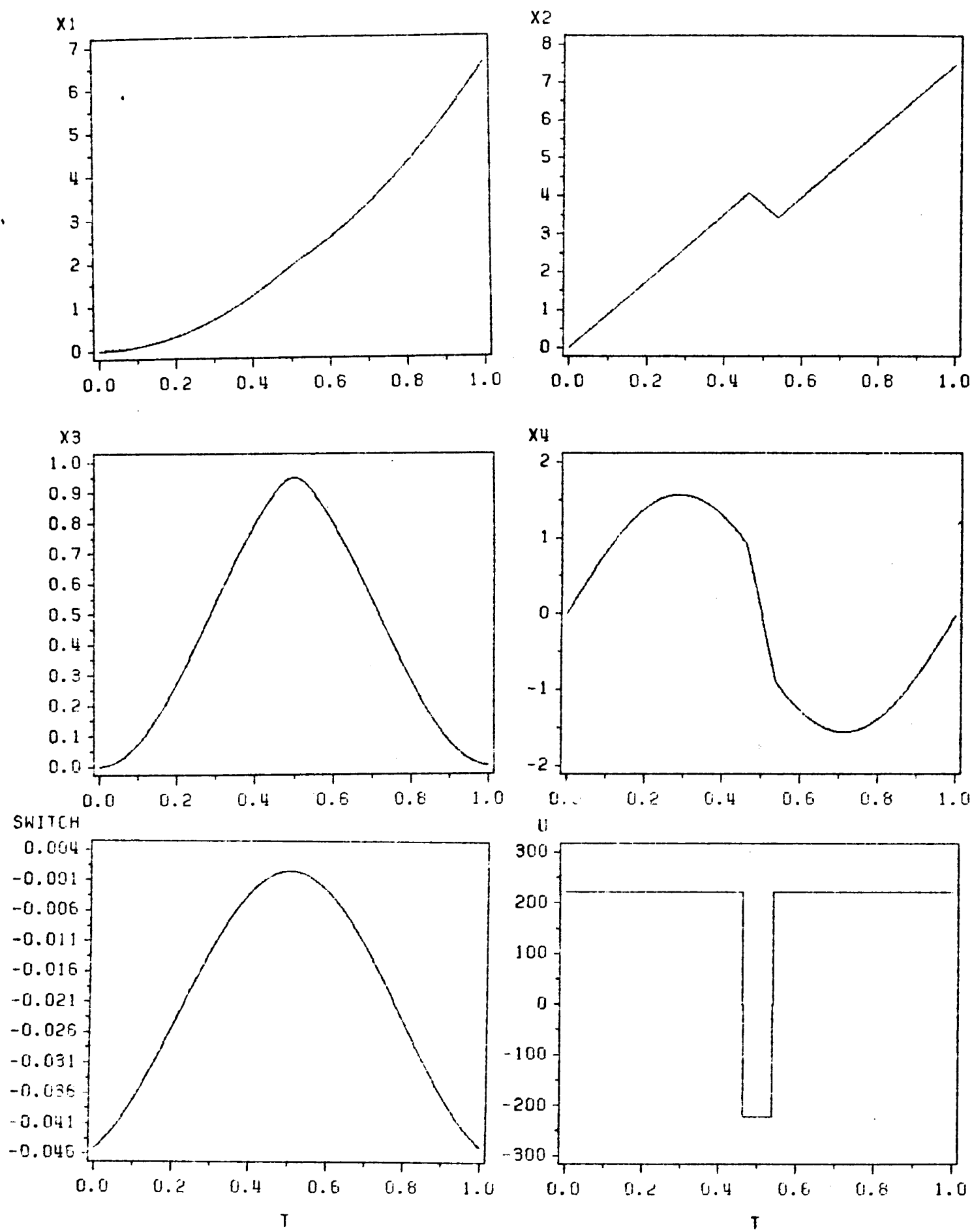

Fig. 10 : One Elastic Mode - Spin-Up 

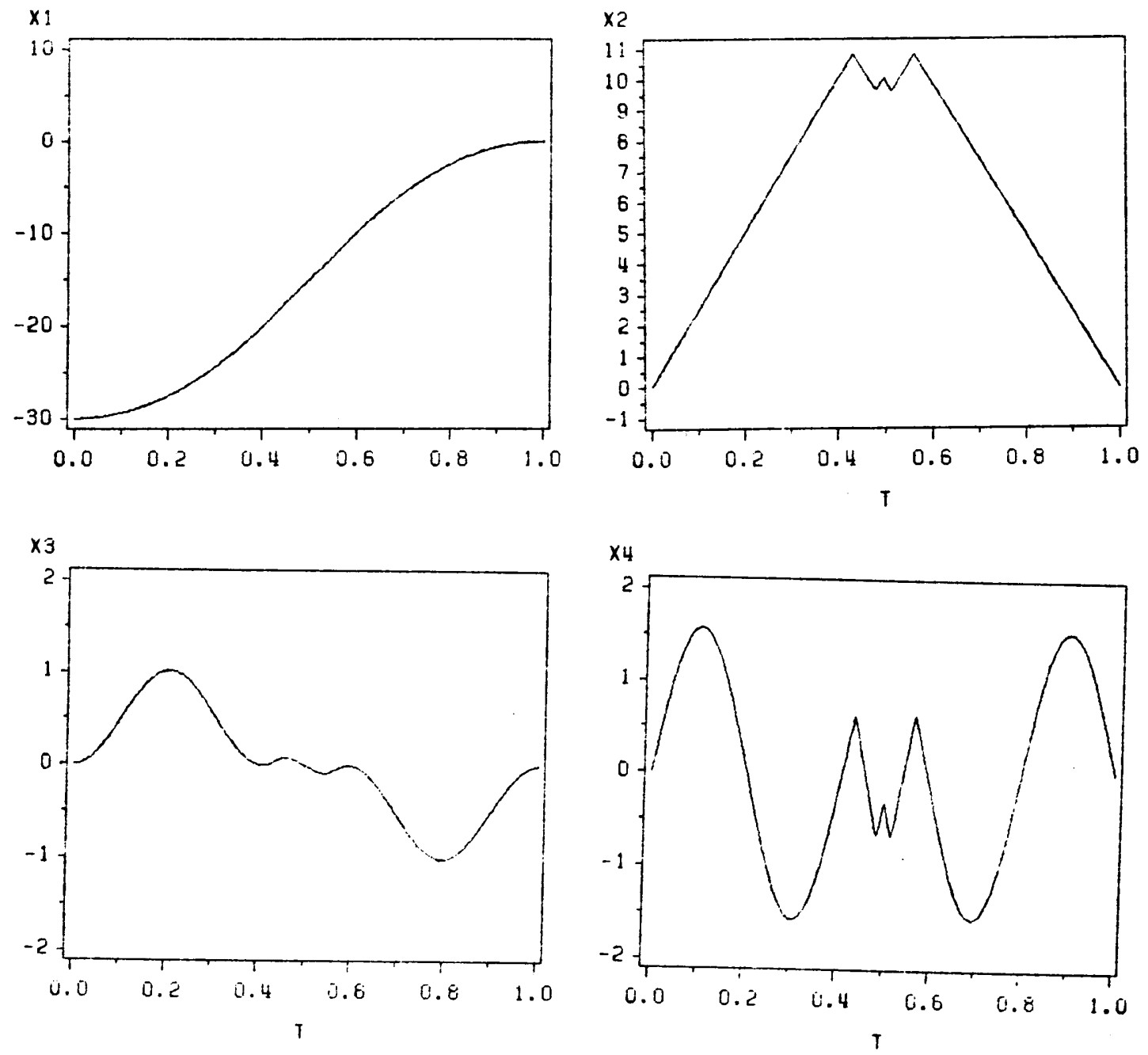

Fig. 11 : Two Elastic Modes - Rest-to-Rest $\left(x^{o}=30\right)$ 

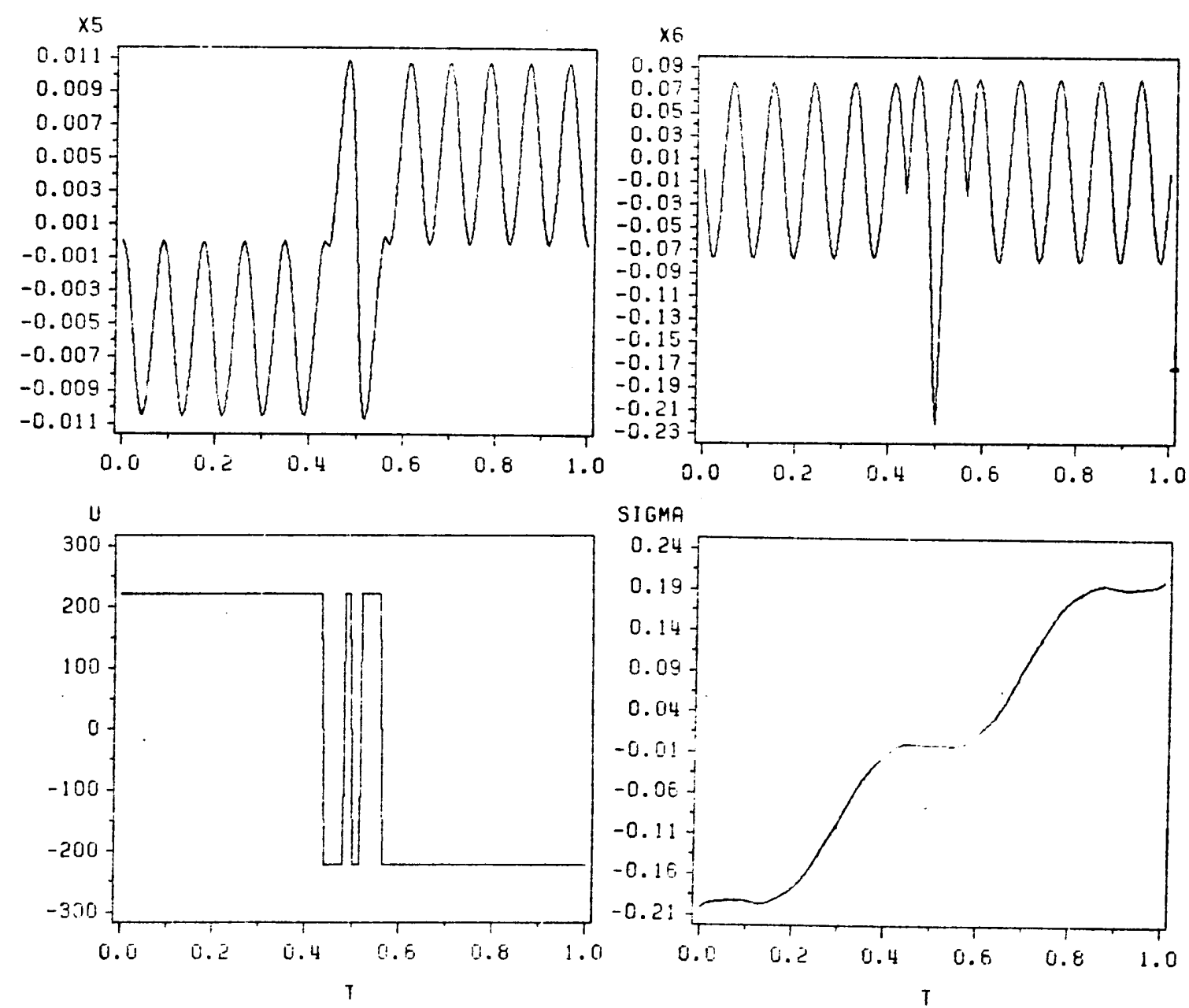

SIGMA

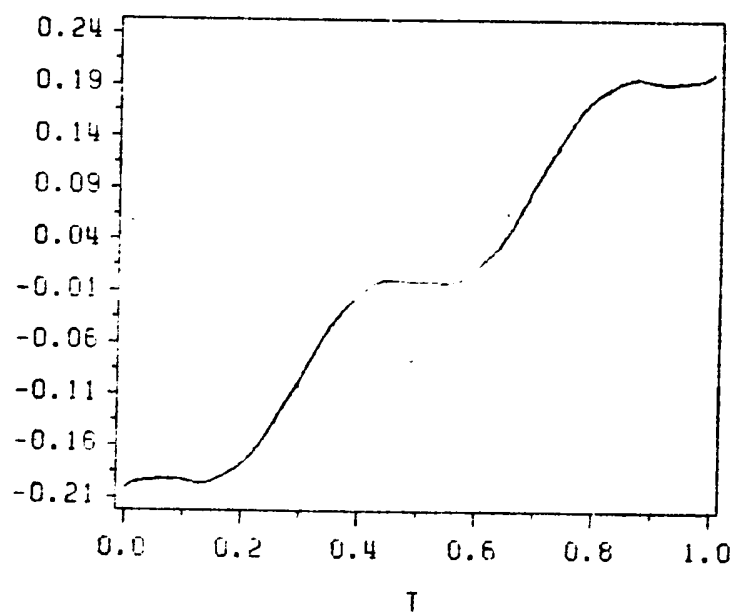

Fig. 11: Two Elastic Modes - Rest-to-Rest $\left(x^{\circ}=30\right)$ (continued) 


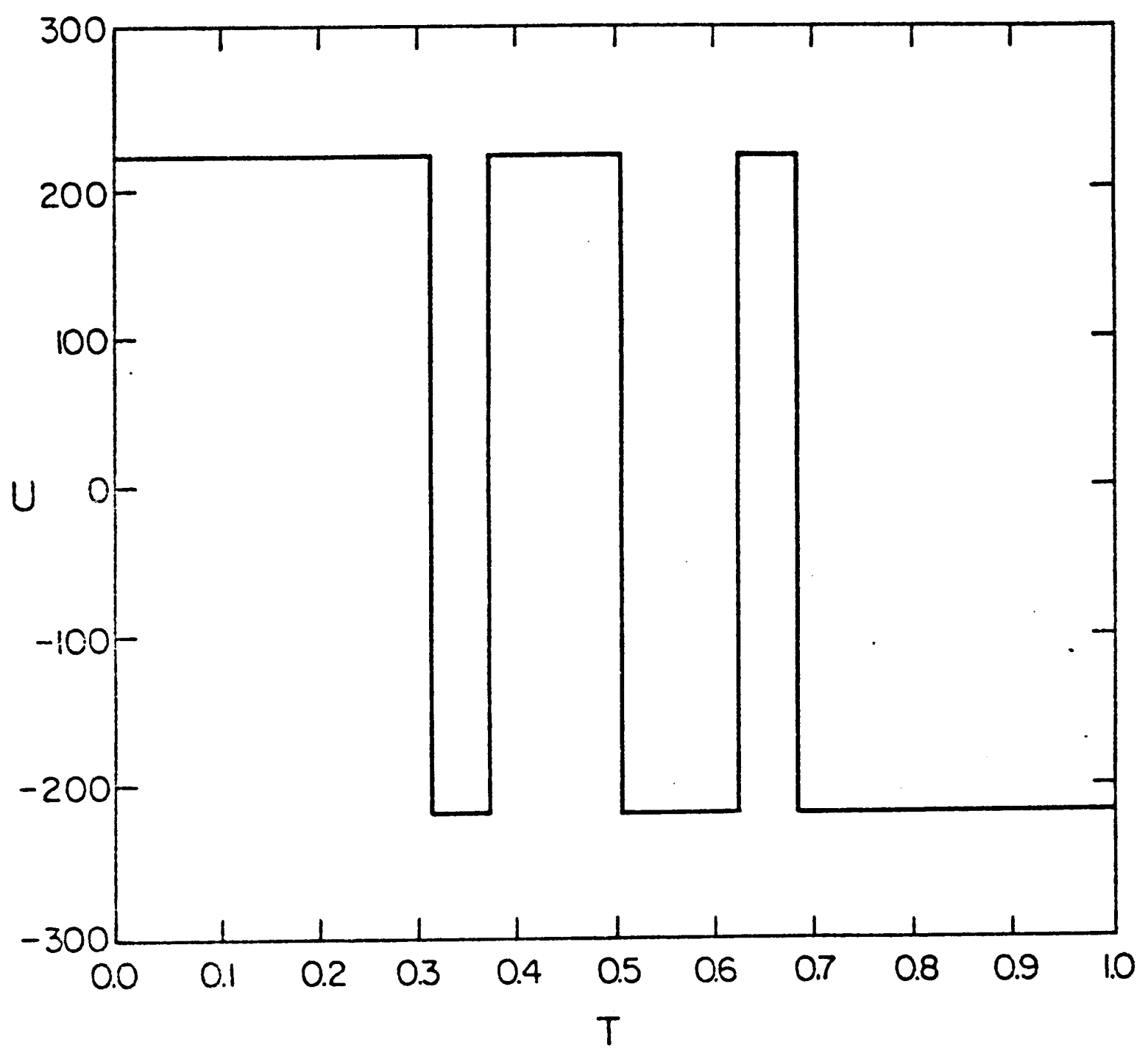

Fig. 12: Suboptimal Solution $\left(x^{o}=10\right)$ 


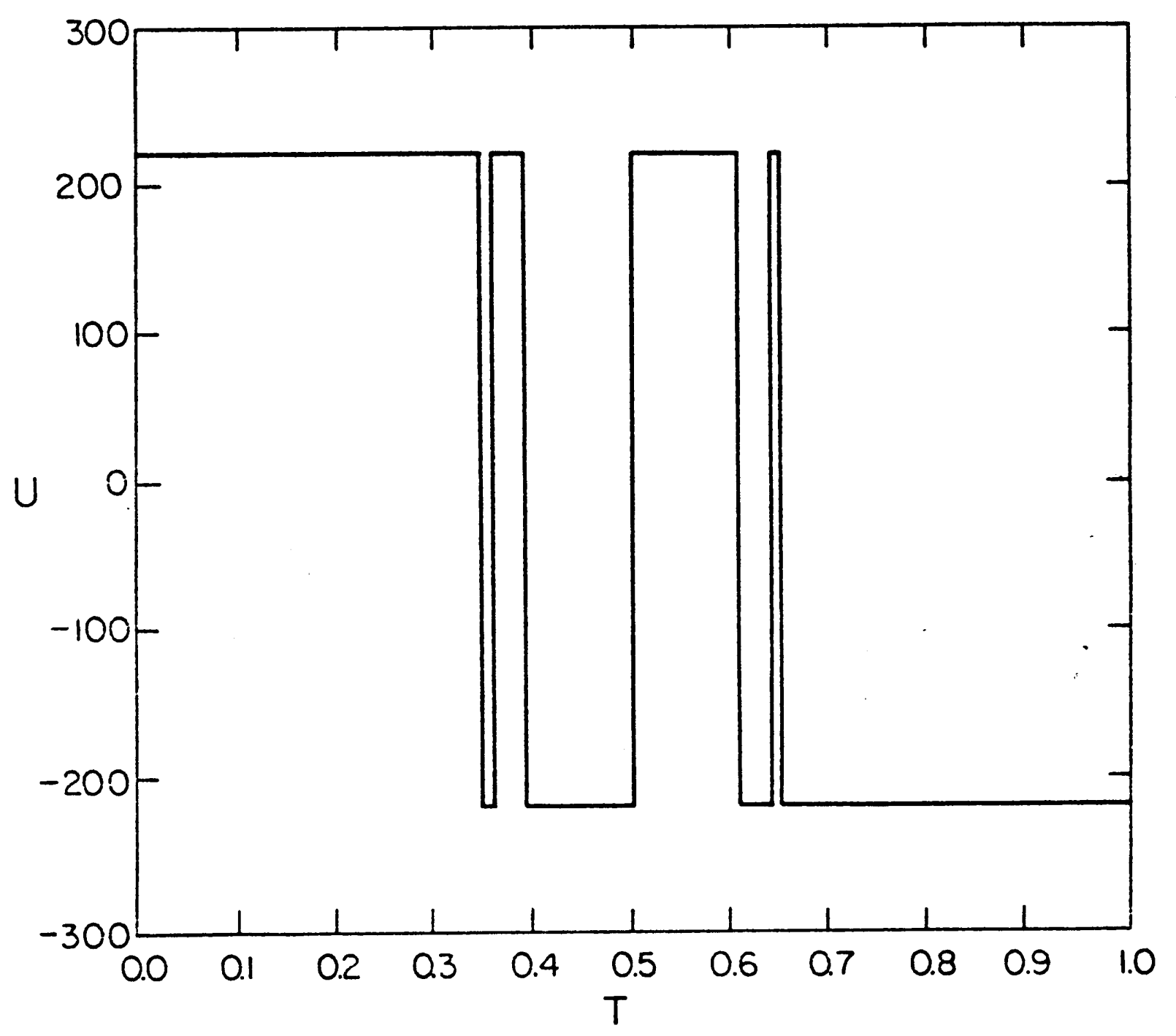

Fig. 13: Optimal Solution $\left(x^{o}=10\right)$ 


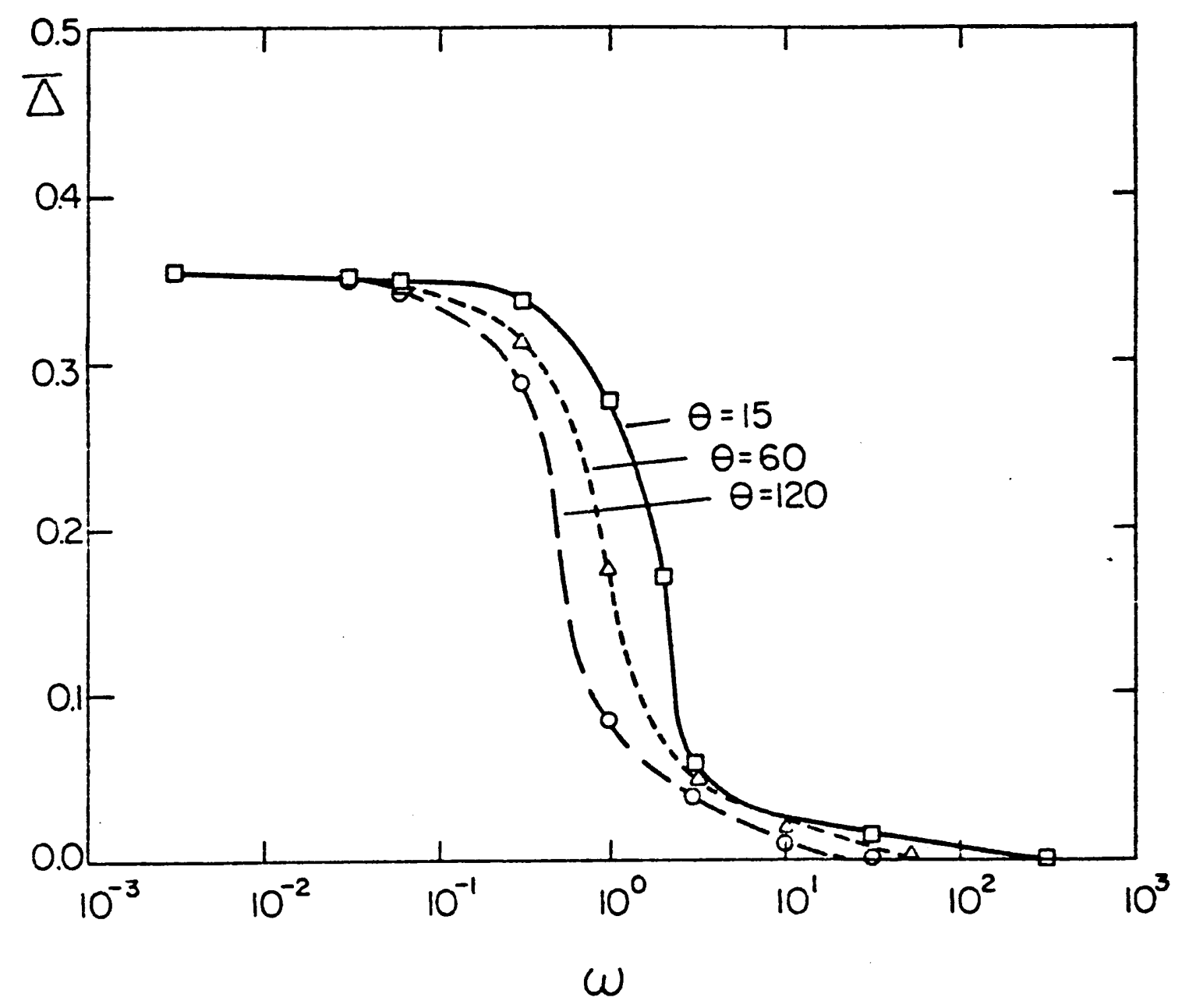

Fig. 14 : Switching Points vs. Frequency 


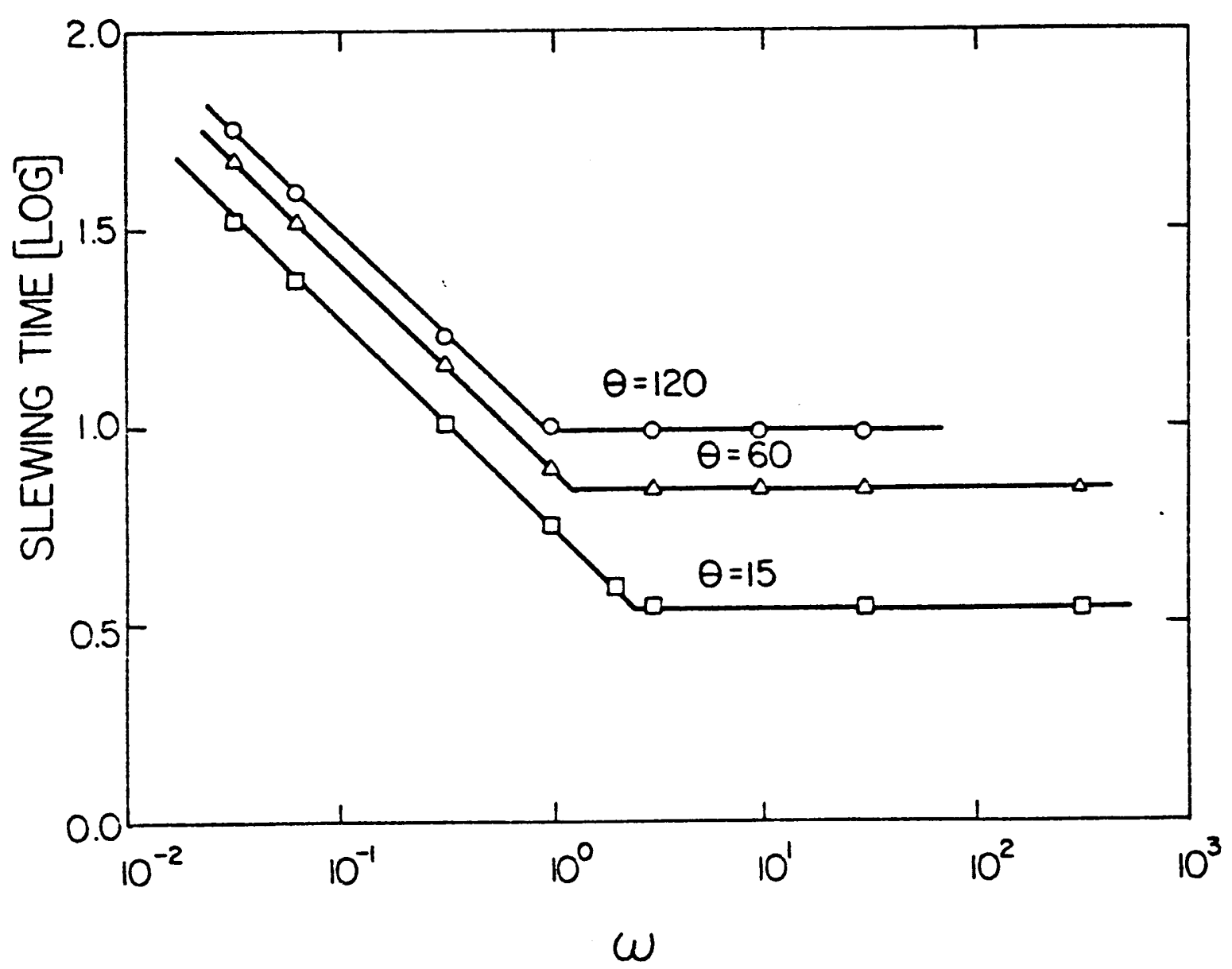

Fig. 15: Slewing Time vs. Frequency 

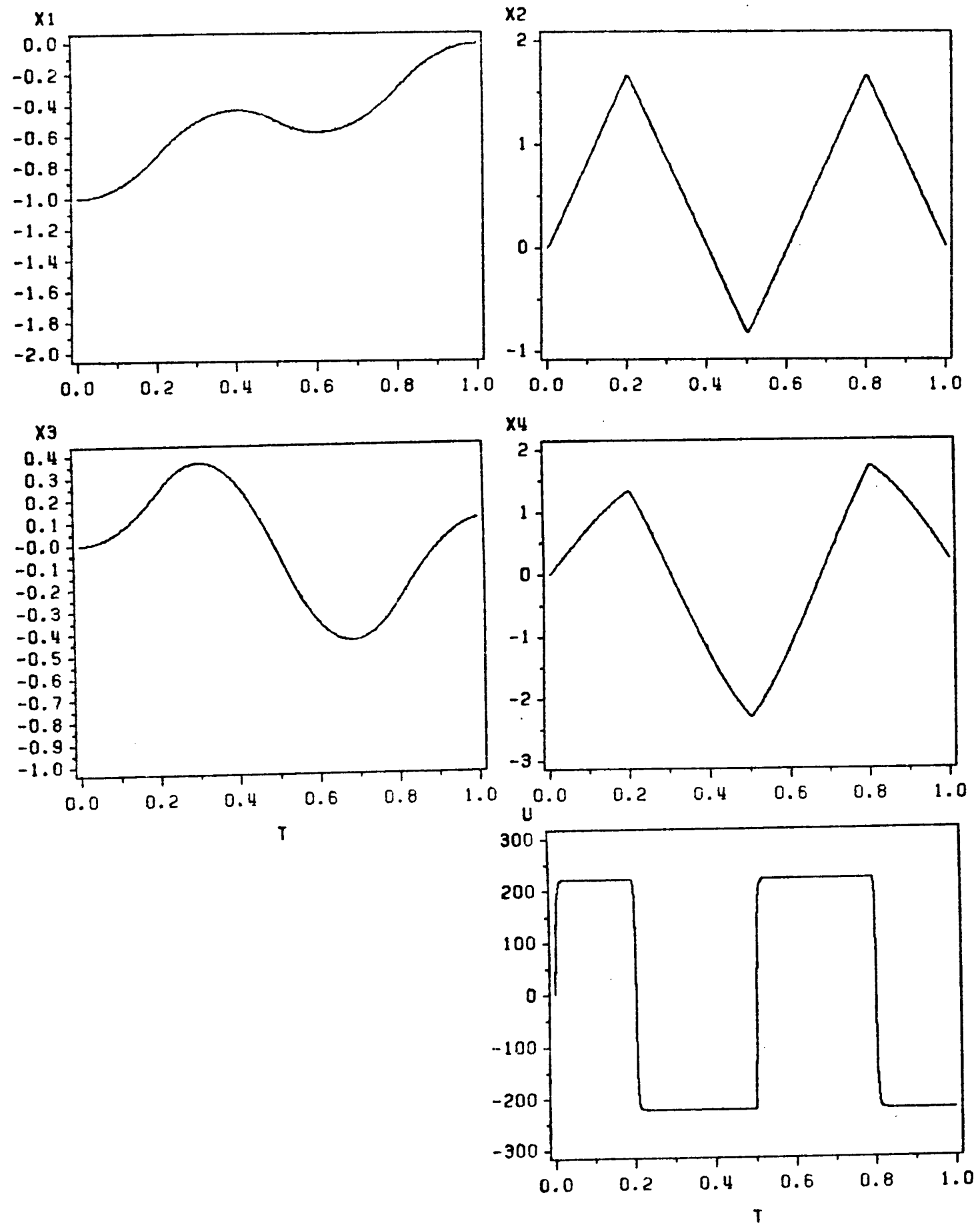

Fig. 16: Approximate Solution $\left(x^{\circ}=1\right)$ 

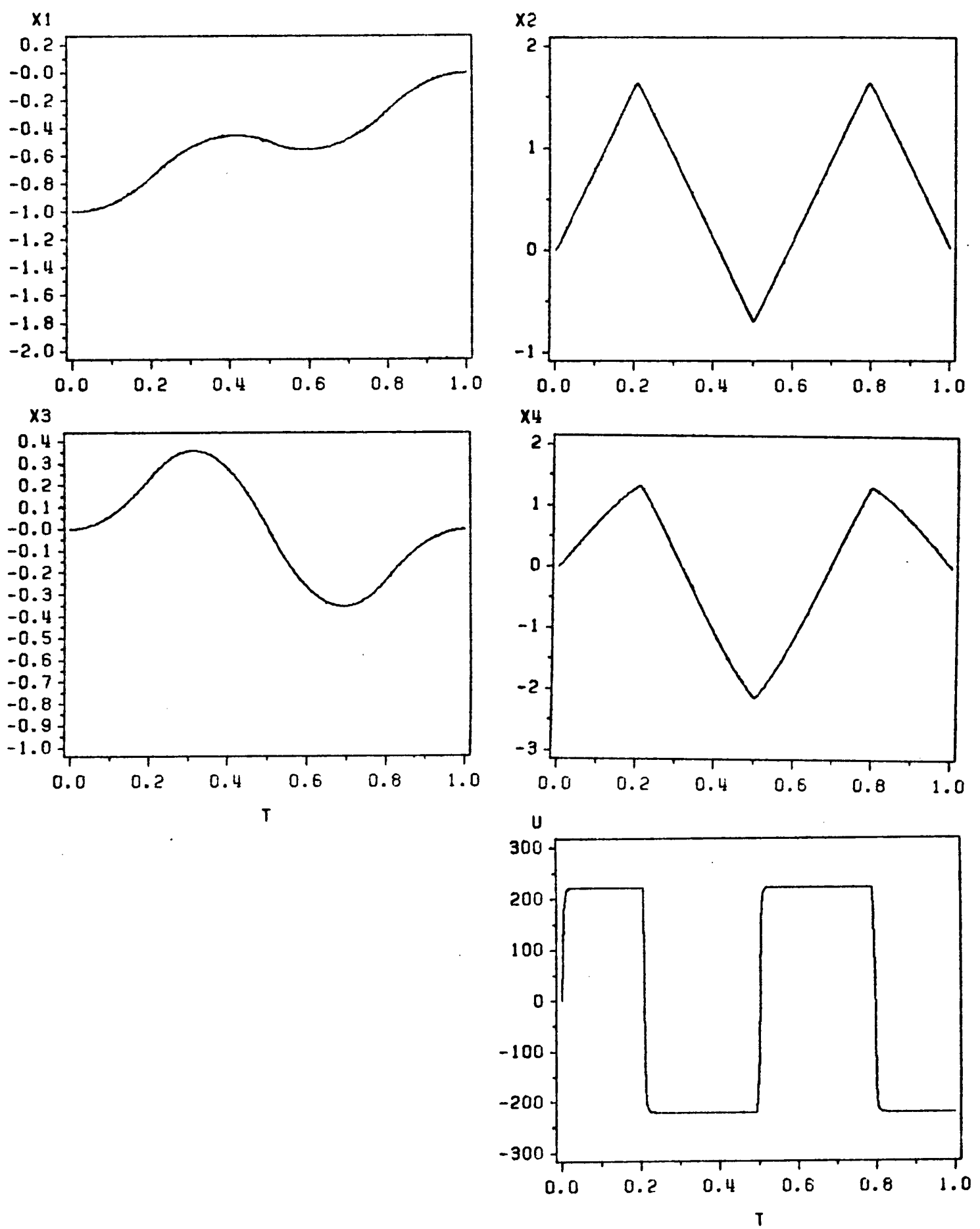

Fig. 17 : Exact Solution $\left(x^{\circ}=1\right)$ 

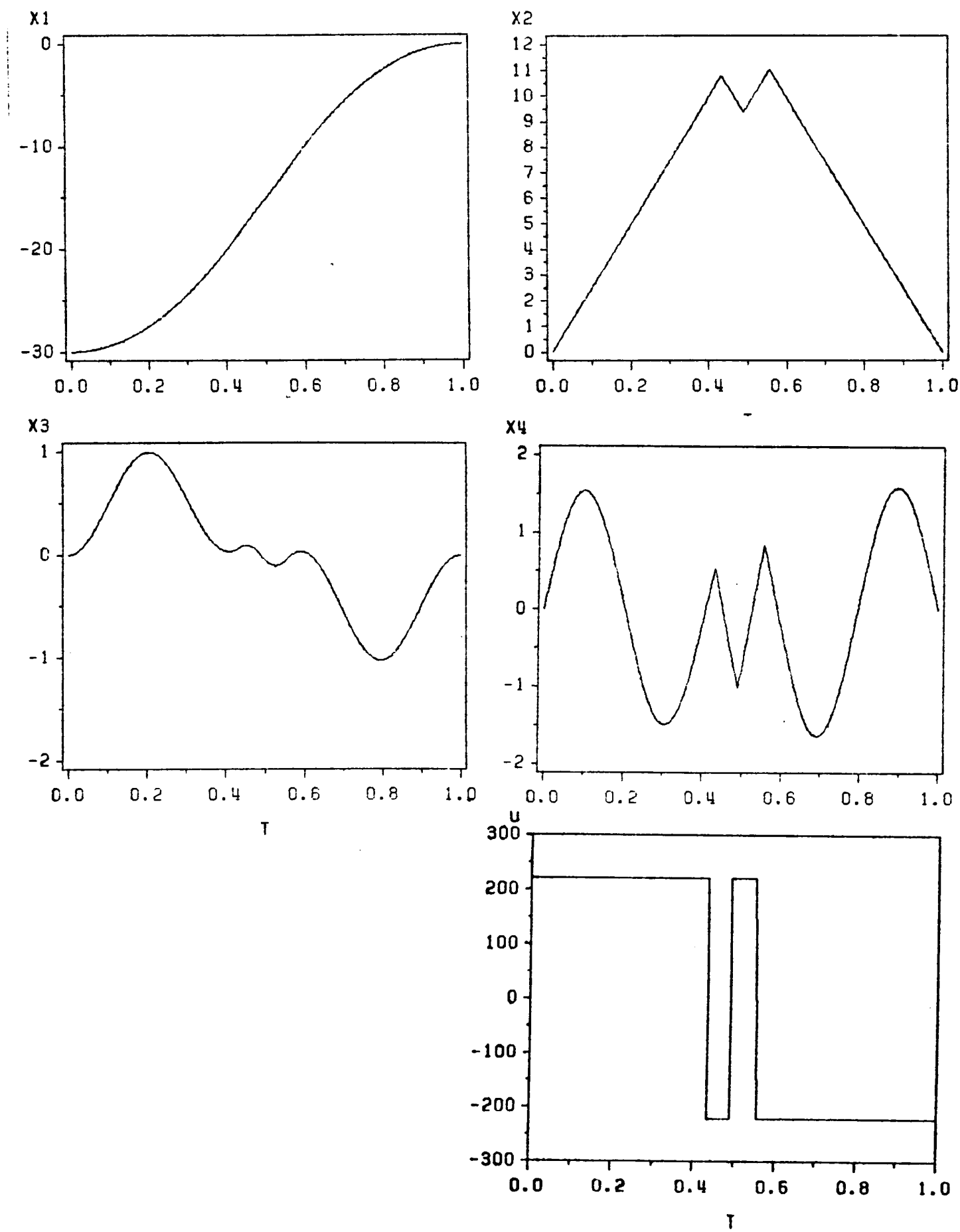

Fig. 18: Damped System 

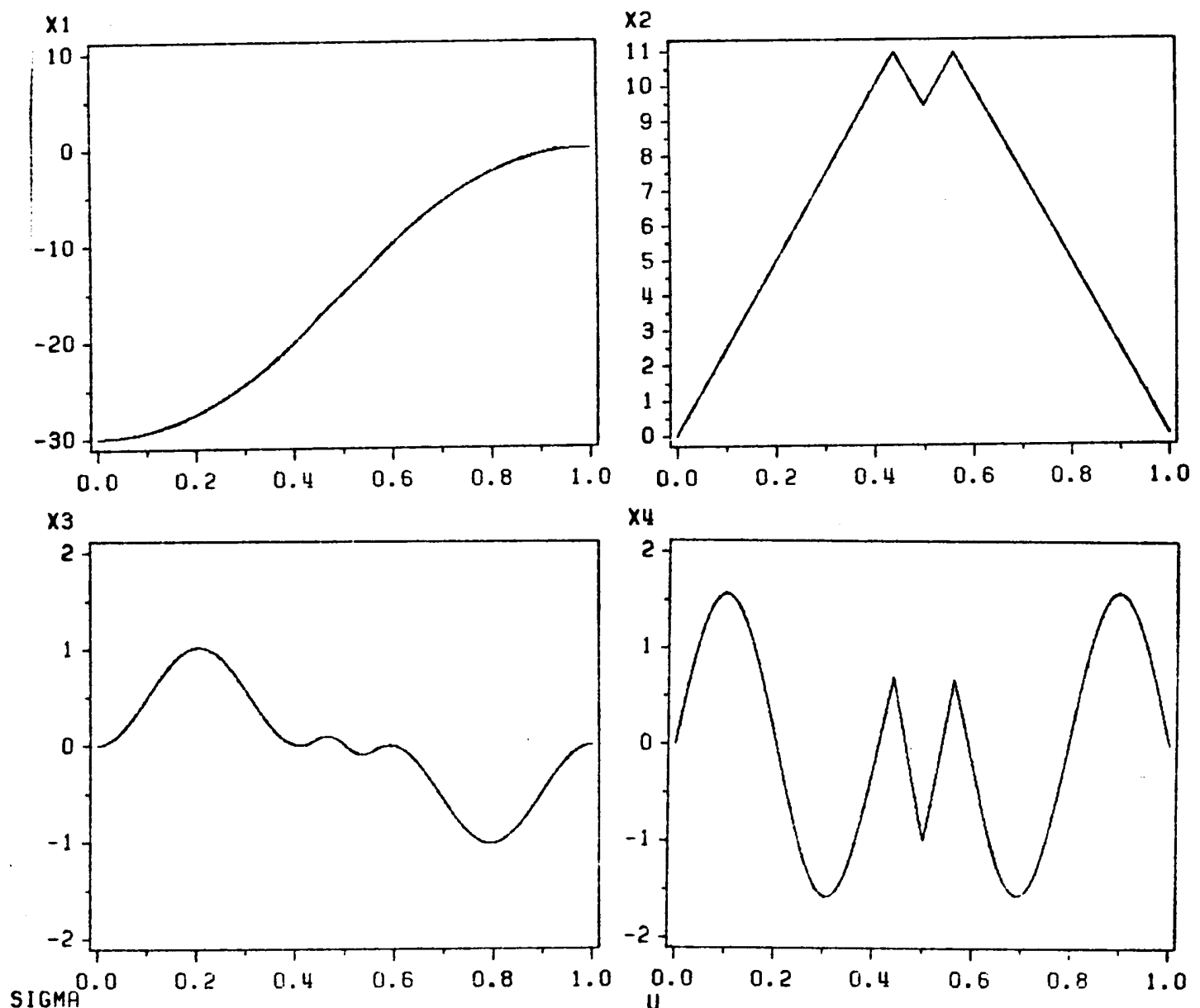

$\times 4$
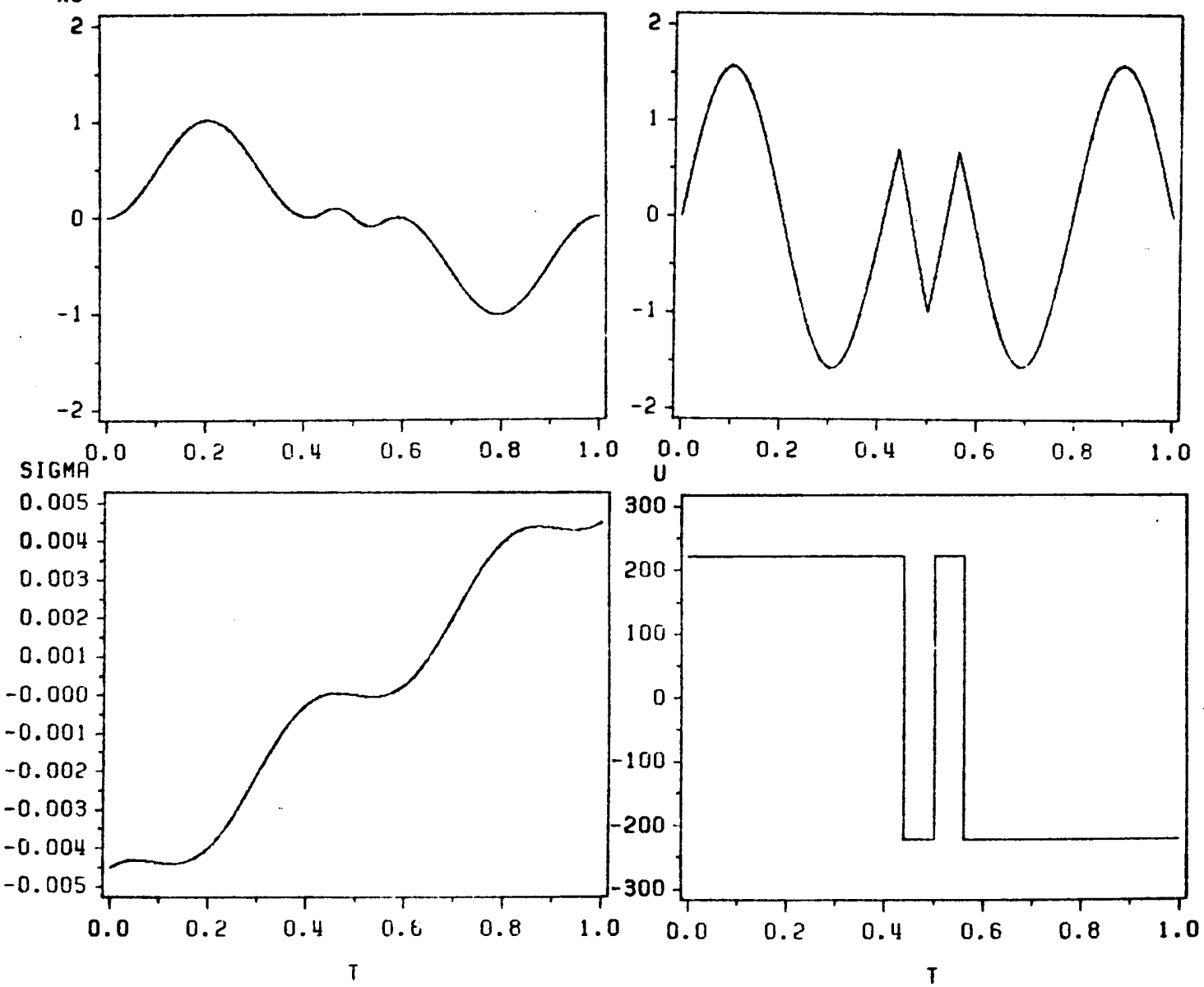

Fig. 19 : Nonlinear System 


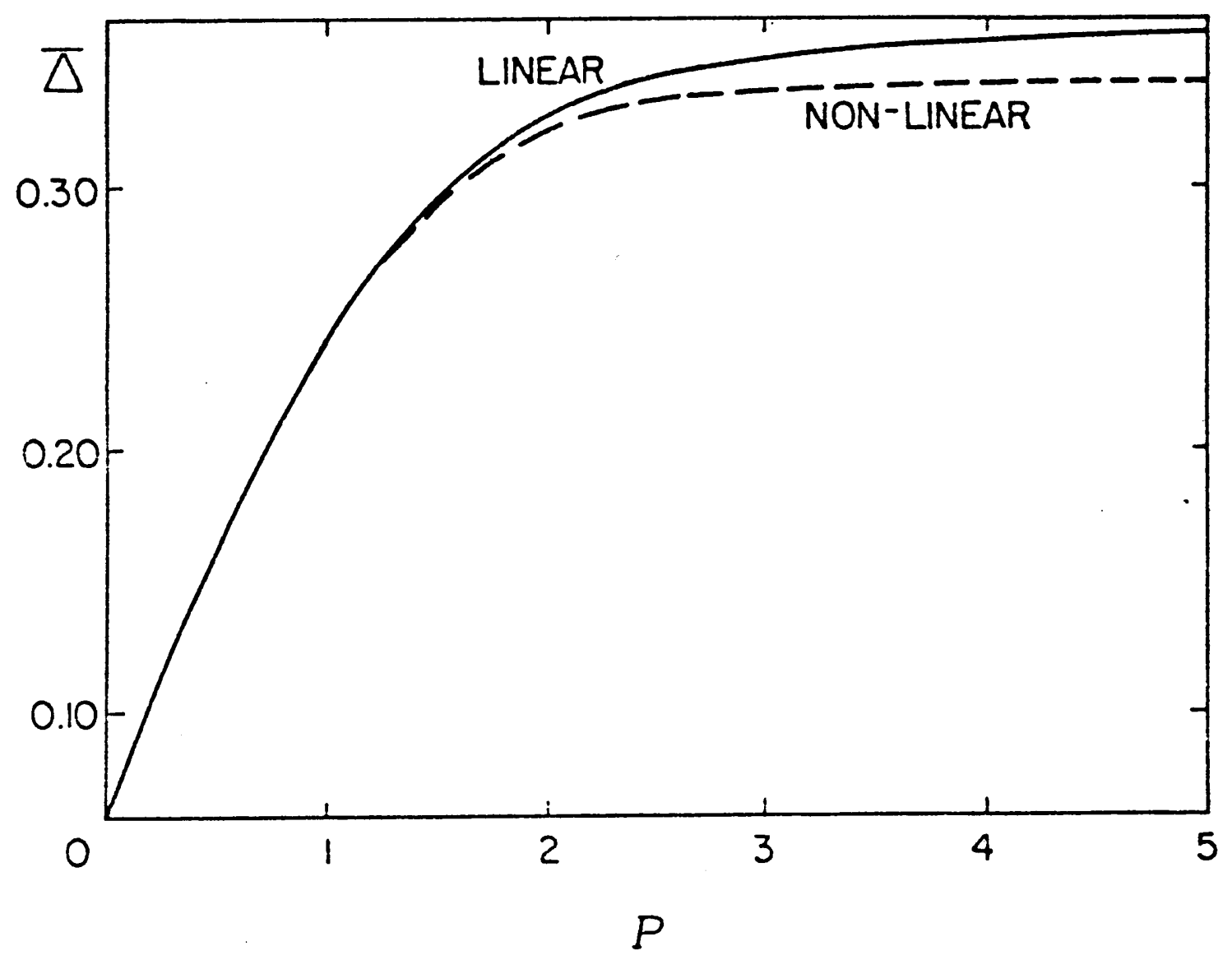

Fig. 20: Switching Points vs. Torque 


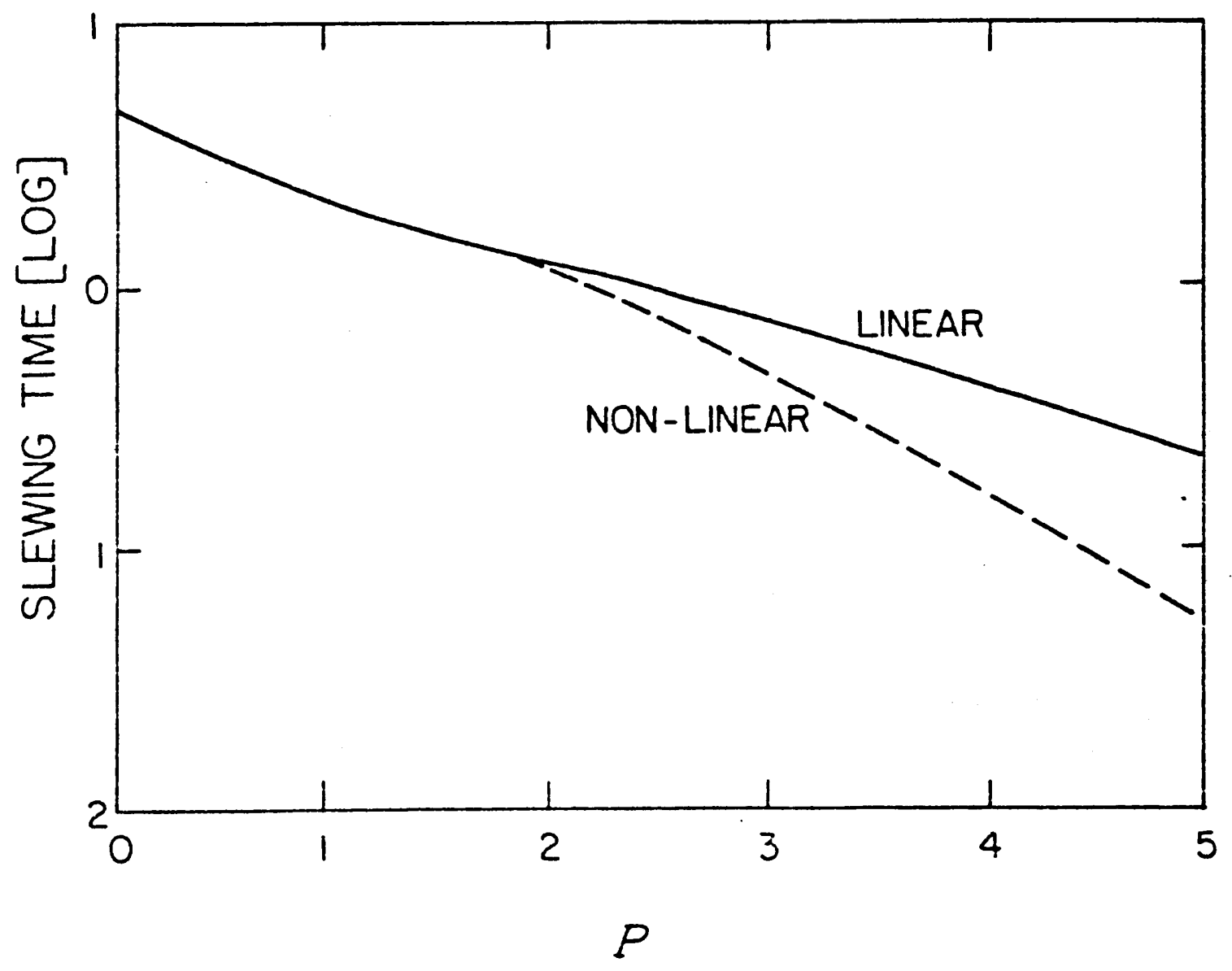

Fig. 21 : Slewing Time vs. Torque 

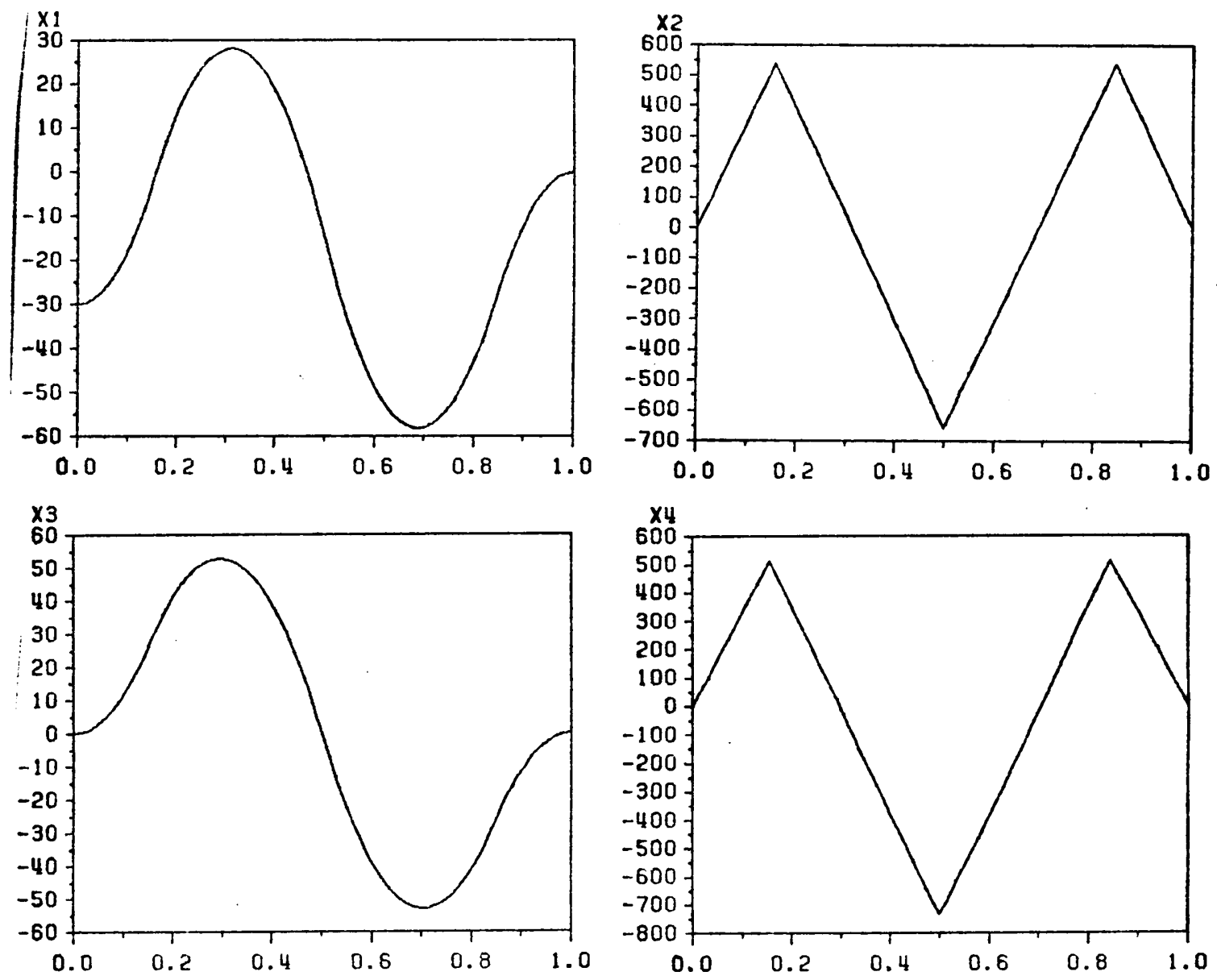

\section{SIGMA}
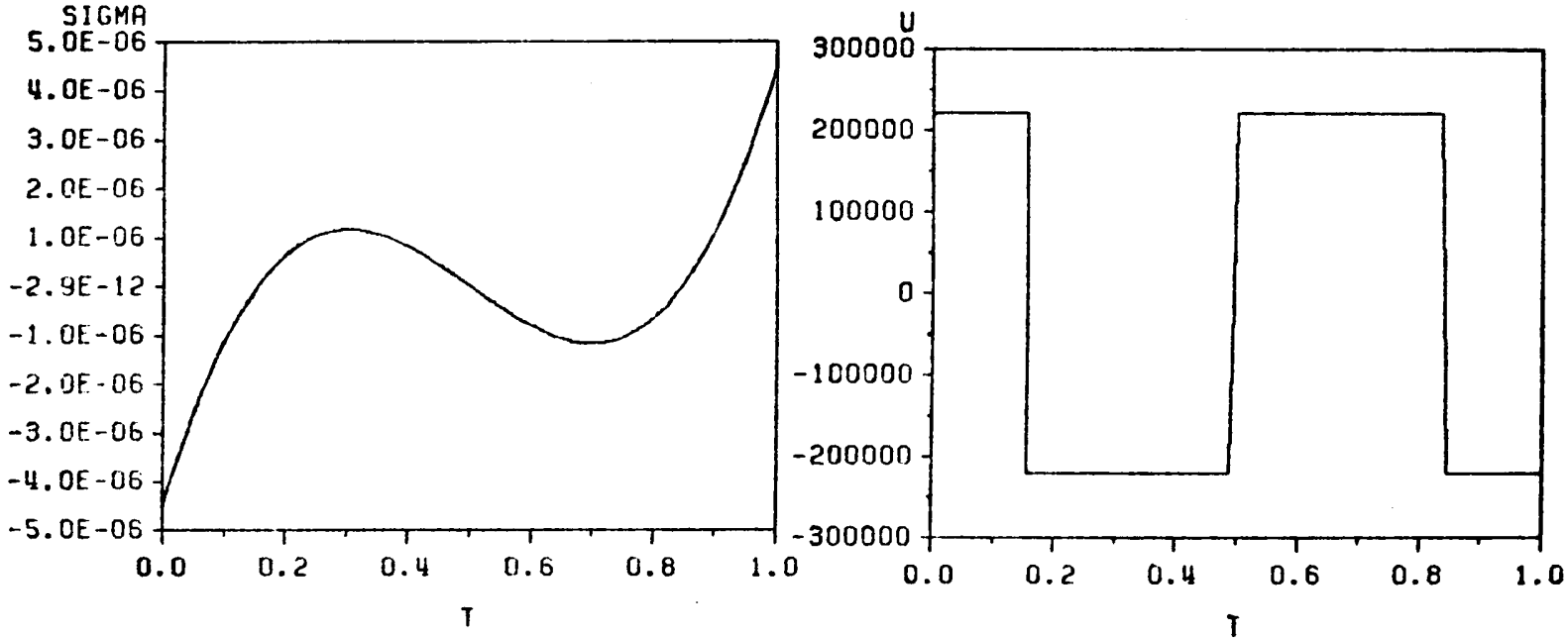

Fig. 22 : Linear solution $(p=3)$ 

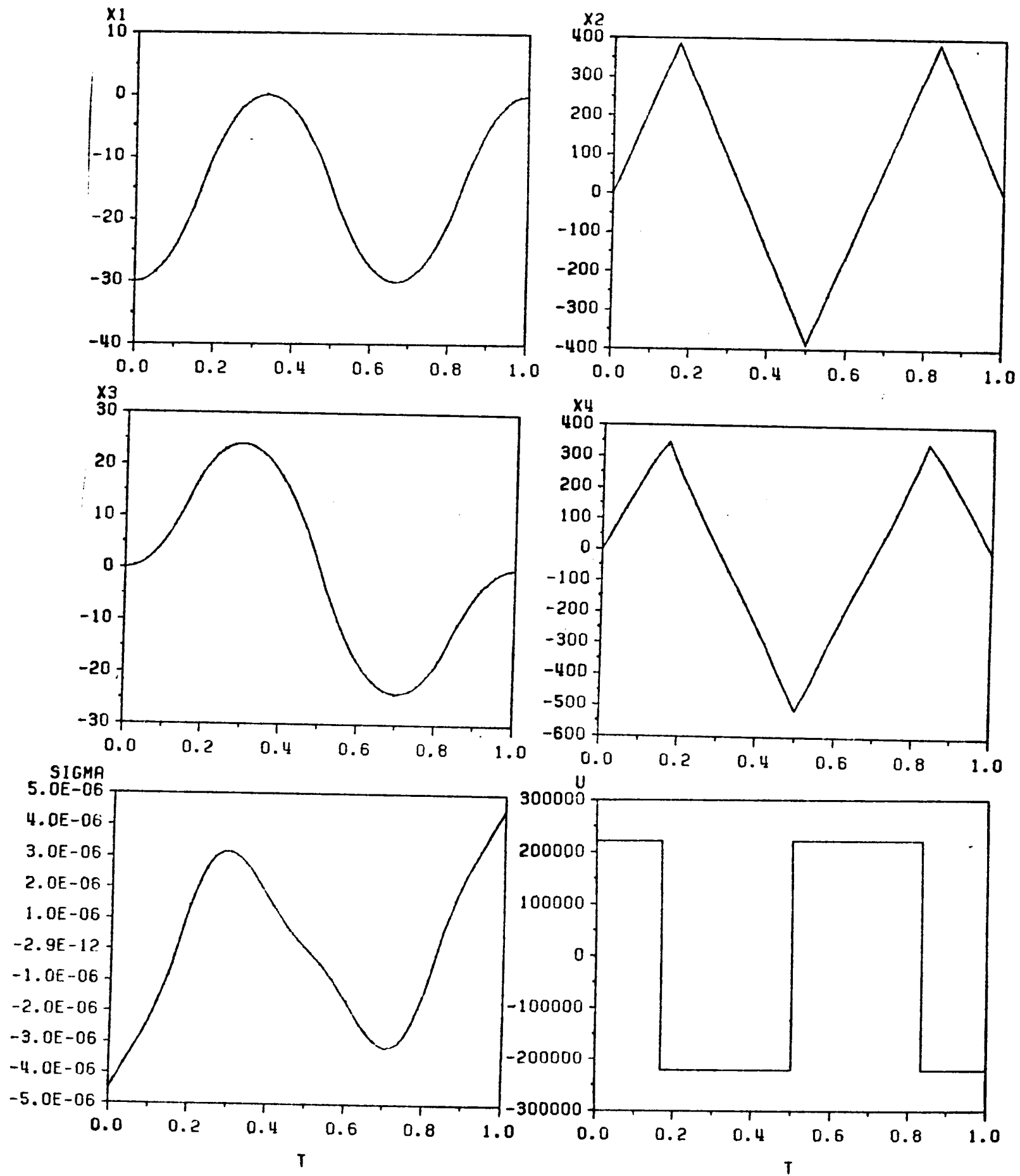

Fig. 23 : Nonlinear solution $(p=3)$ 

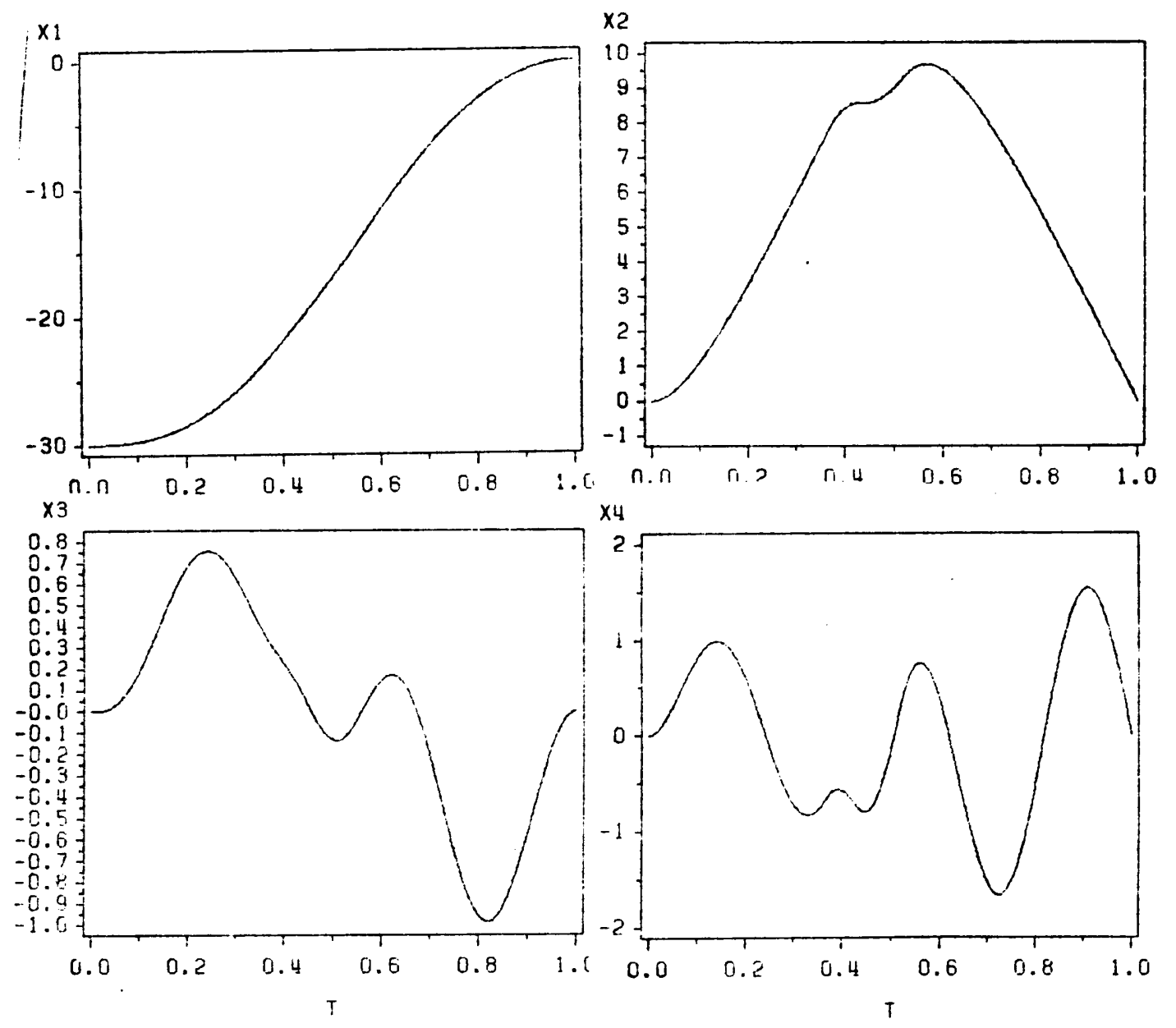

Fig. 24 : Nonideal Actuator $(\tau=0.5)$ 

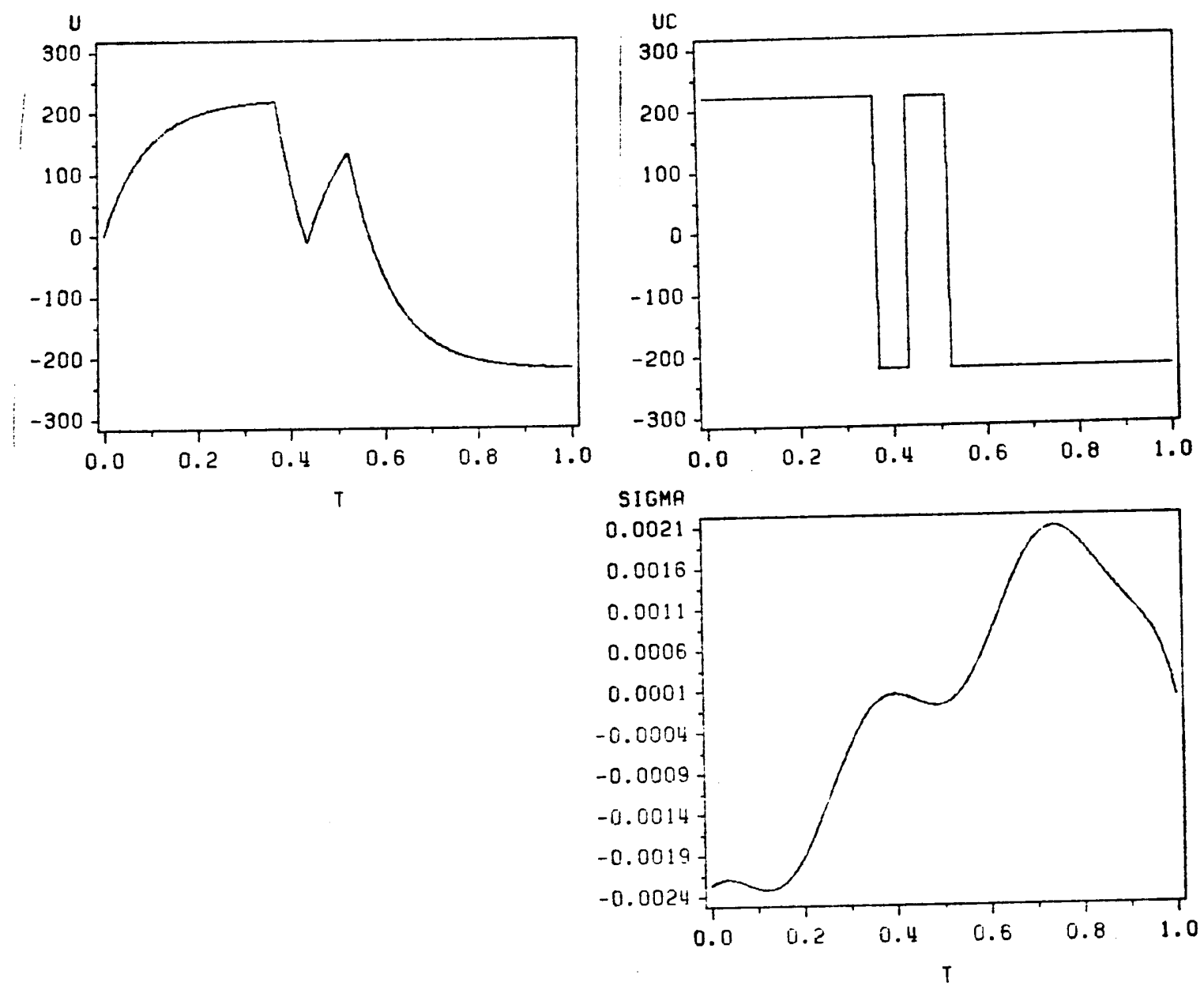

Fig. 24 : Nonideal Actuator $(\tau=0.5)$ (continued) 

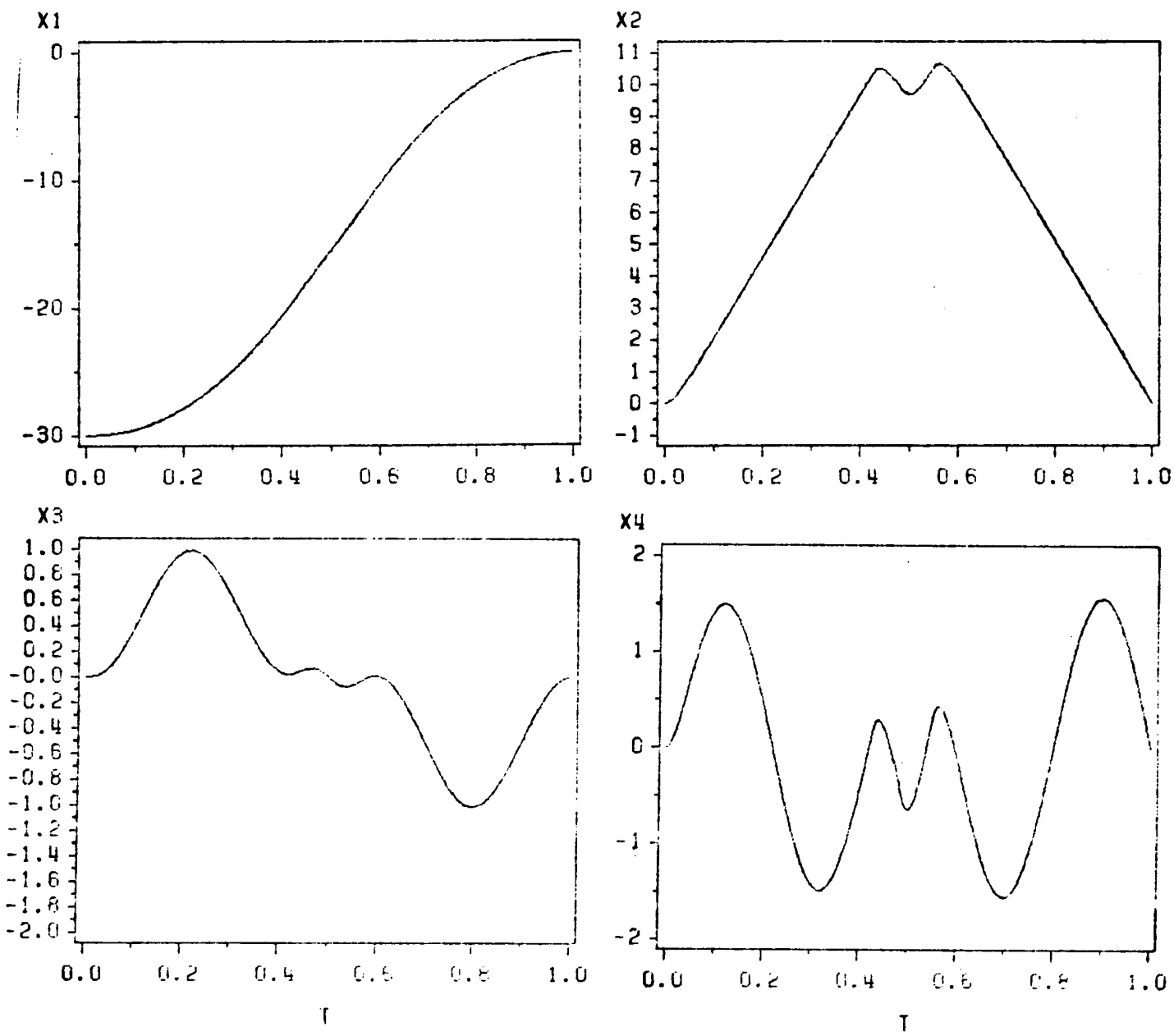

Fig. 25 : Nonideal Actuator $(\tau=0.1)$ 

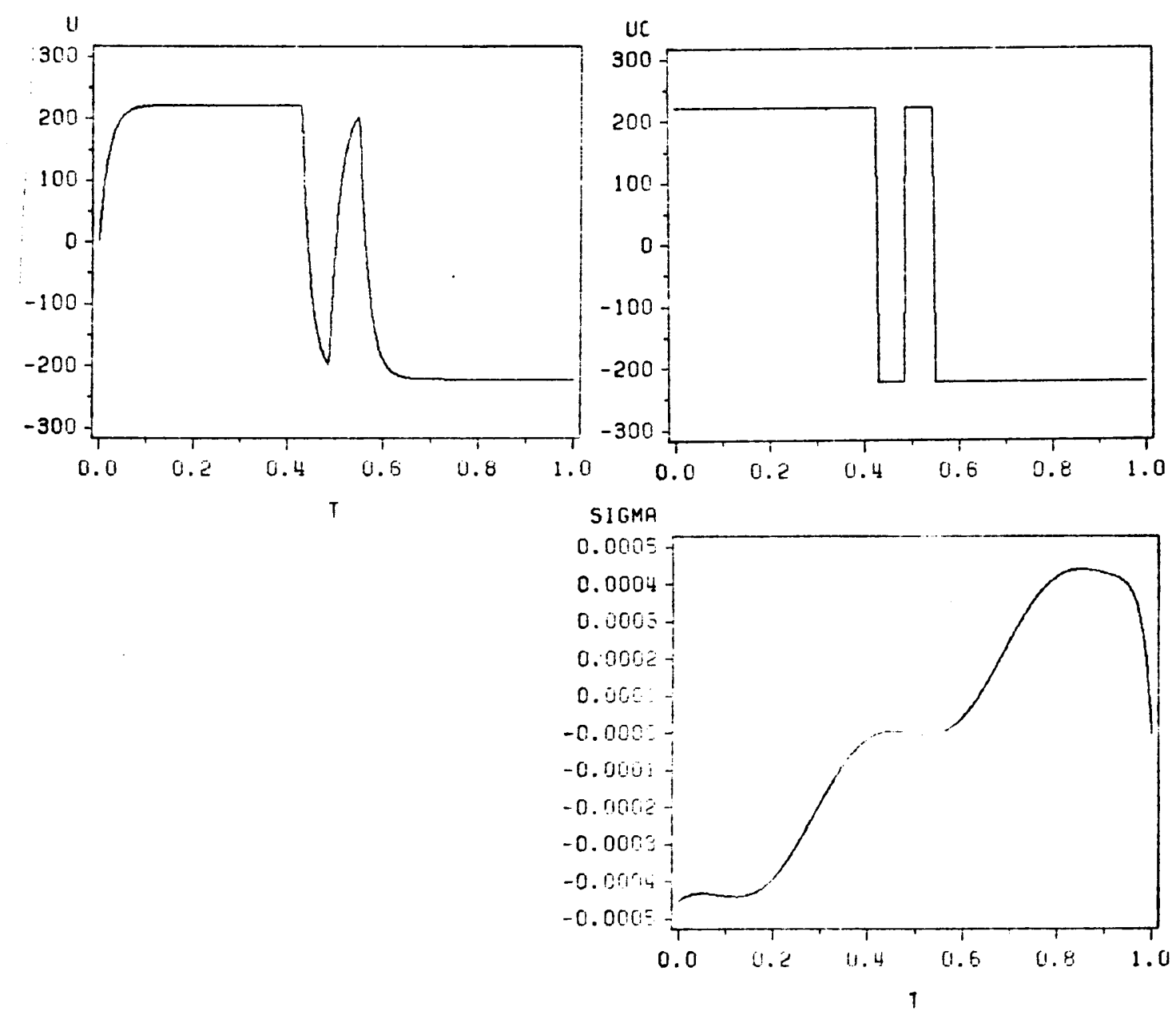

Fig. 25 : Nonideal Actuator $(\tau=0.1)$ (continued) 


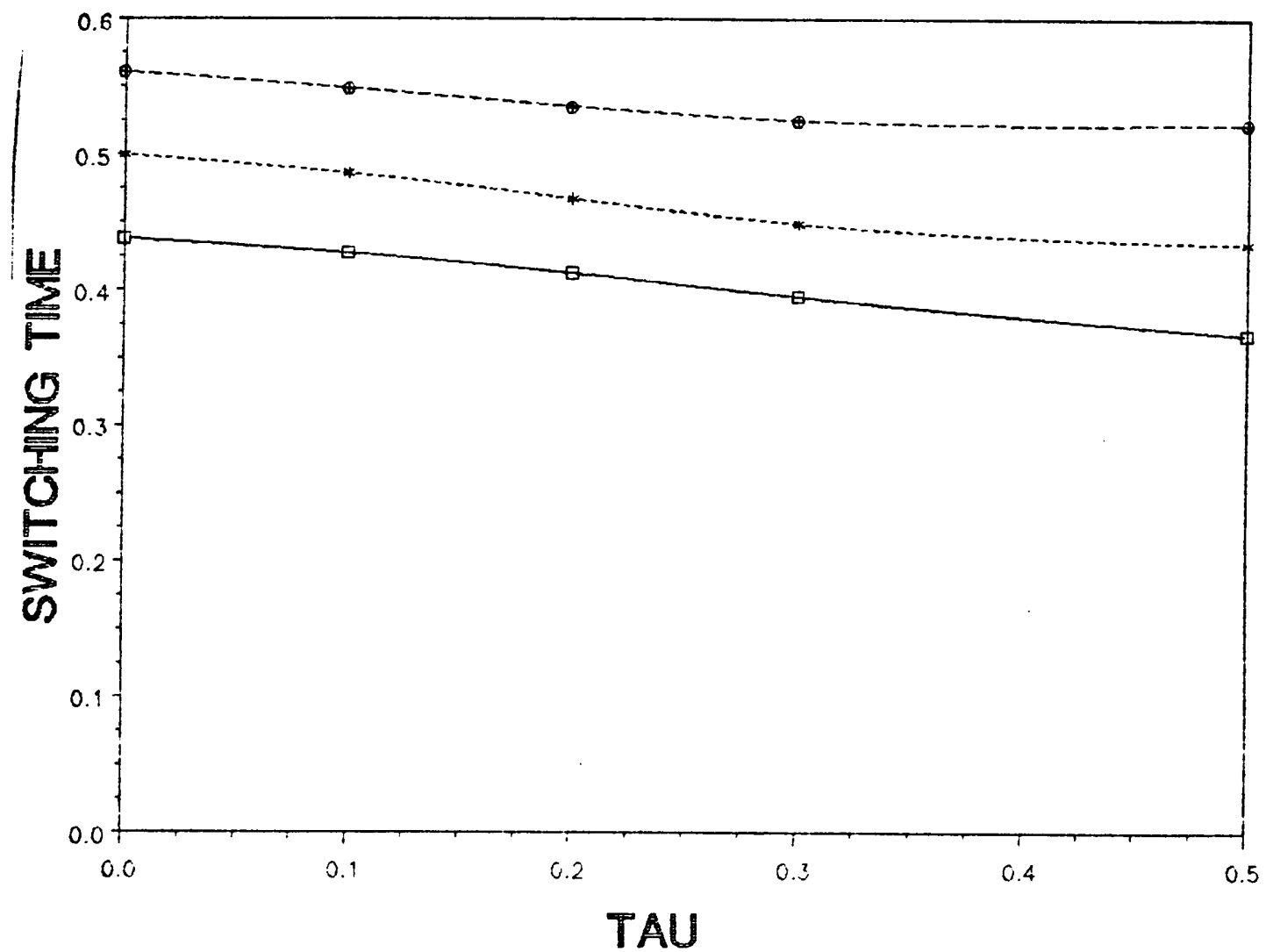

Fig. 26 : Switching Points vs. $\tau$ 


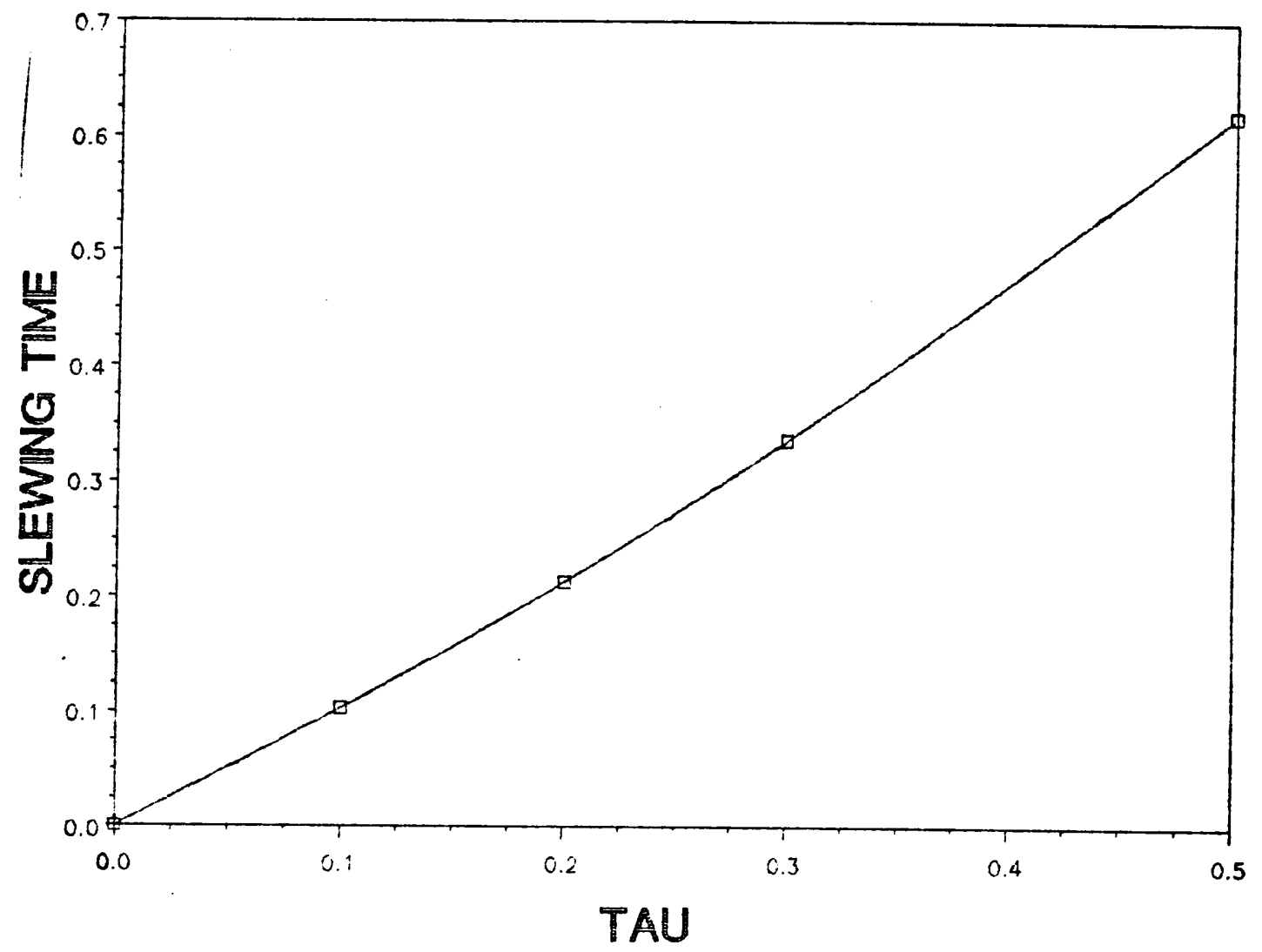

Fig. 27 : Slewing Time vs. $\tau$ 


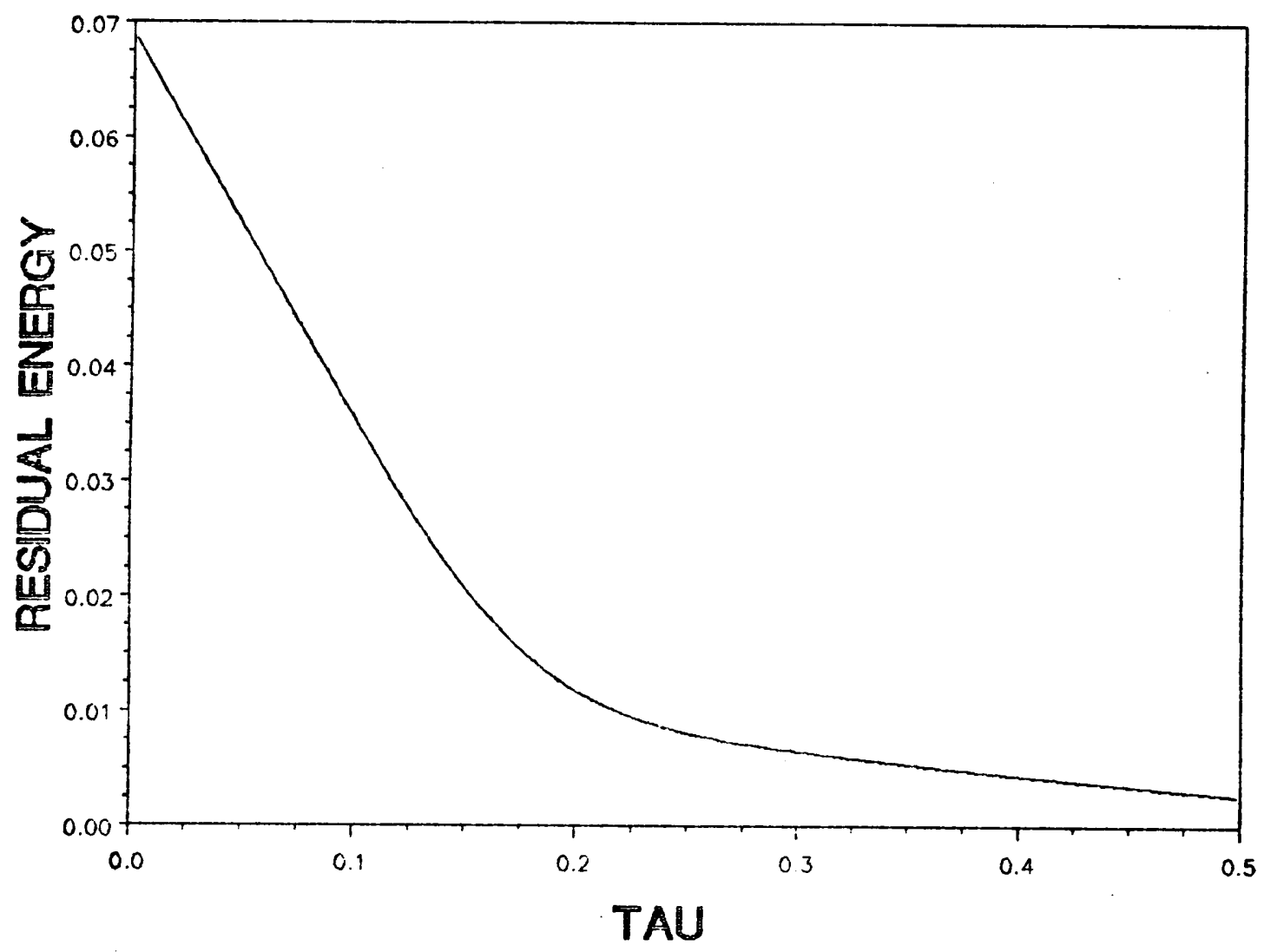

Fig. 28 : Residual Energy vs. $\tau$ 


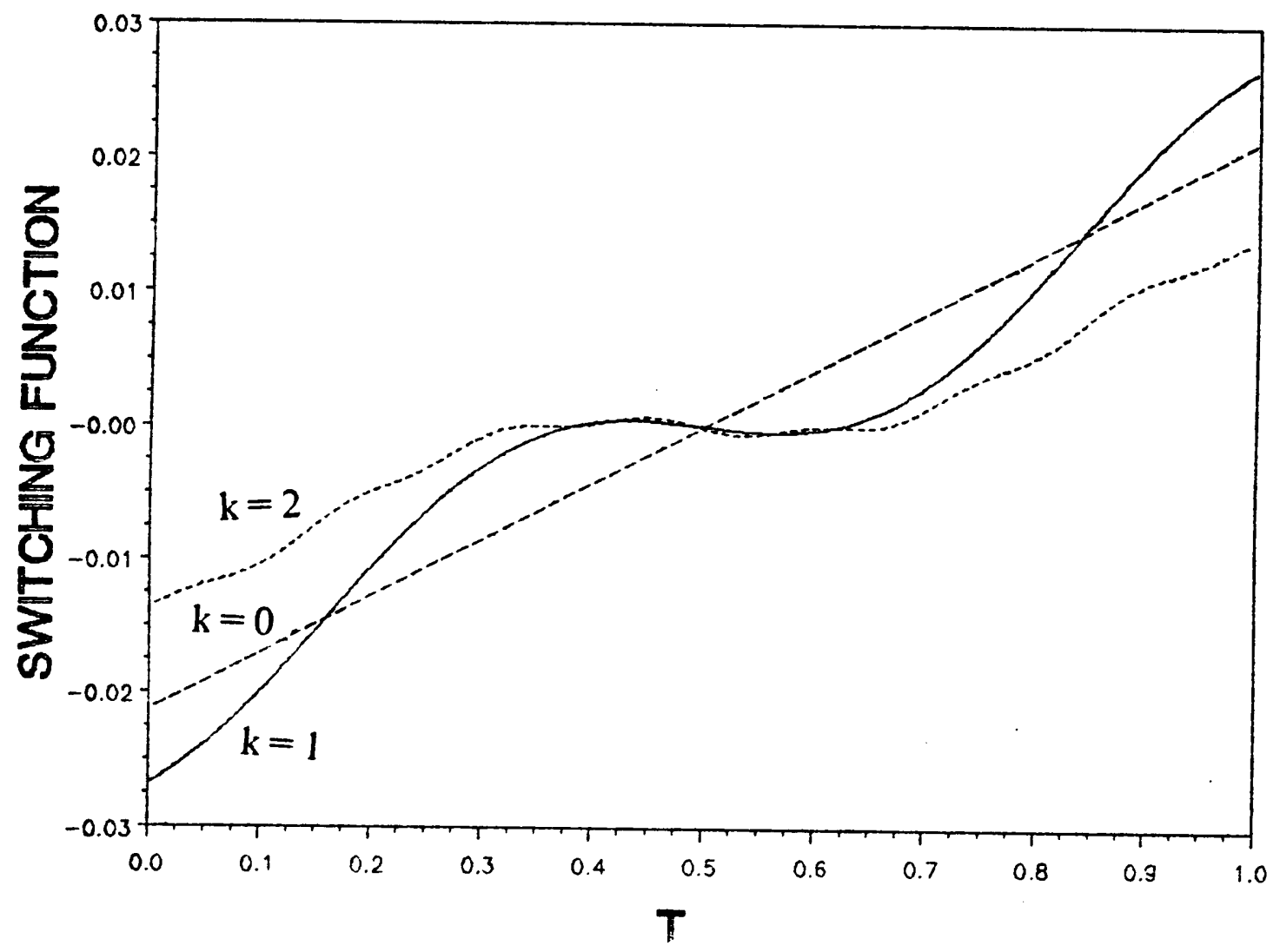

Fig. 29: Switching Function $\left(x^{\circ}=10\right)$ 


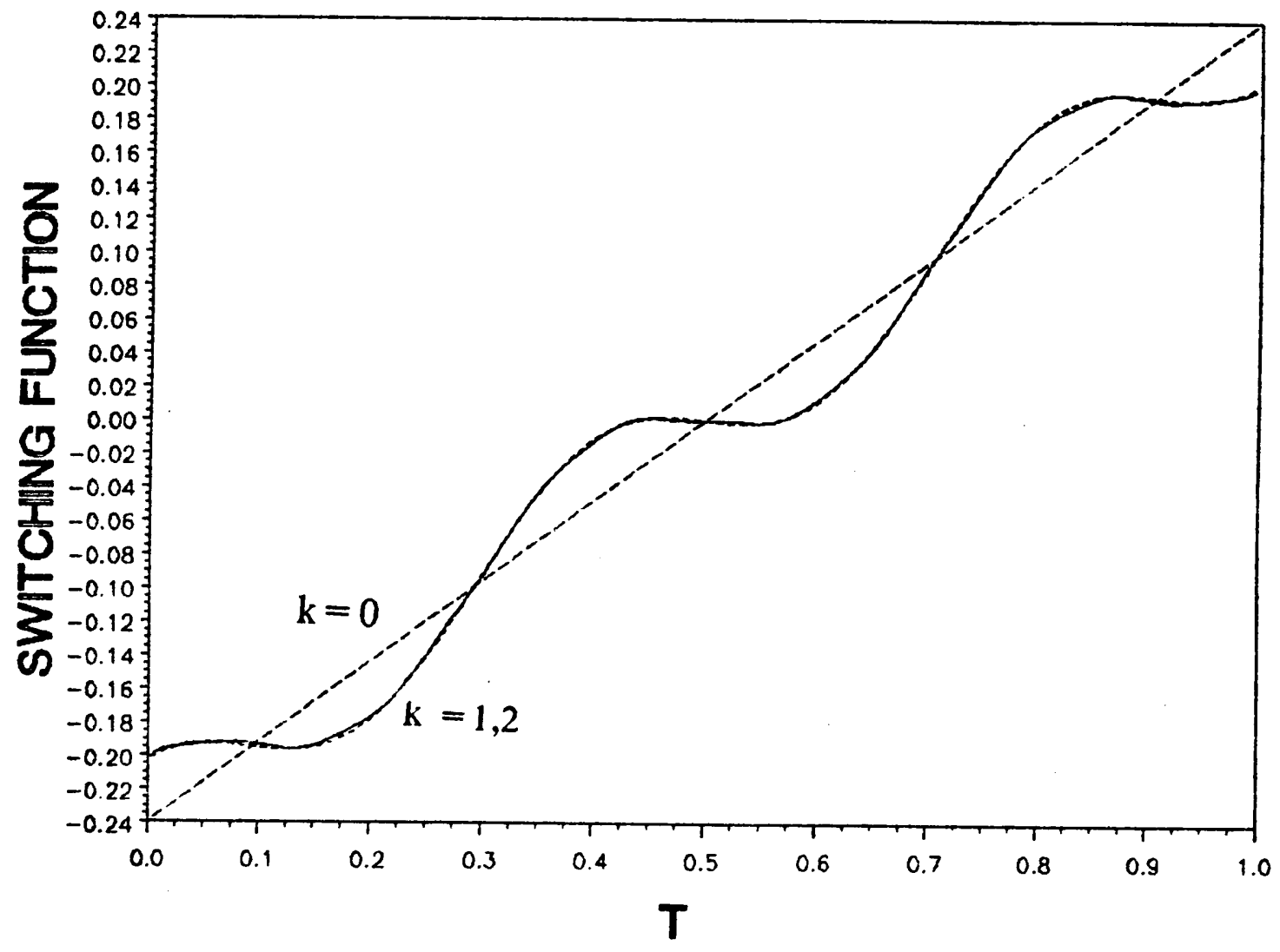

Fig. 30 : Switching Function $\left(x^{\circ}=30\right)$ 

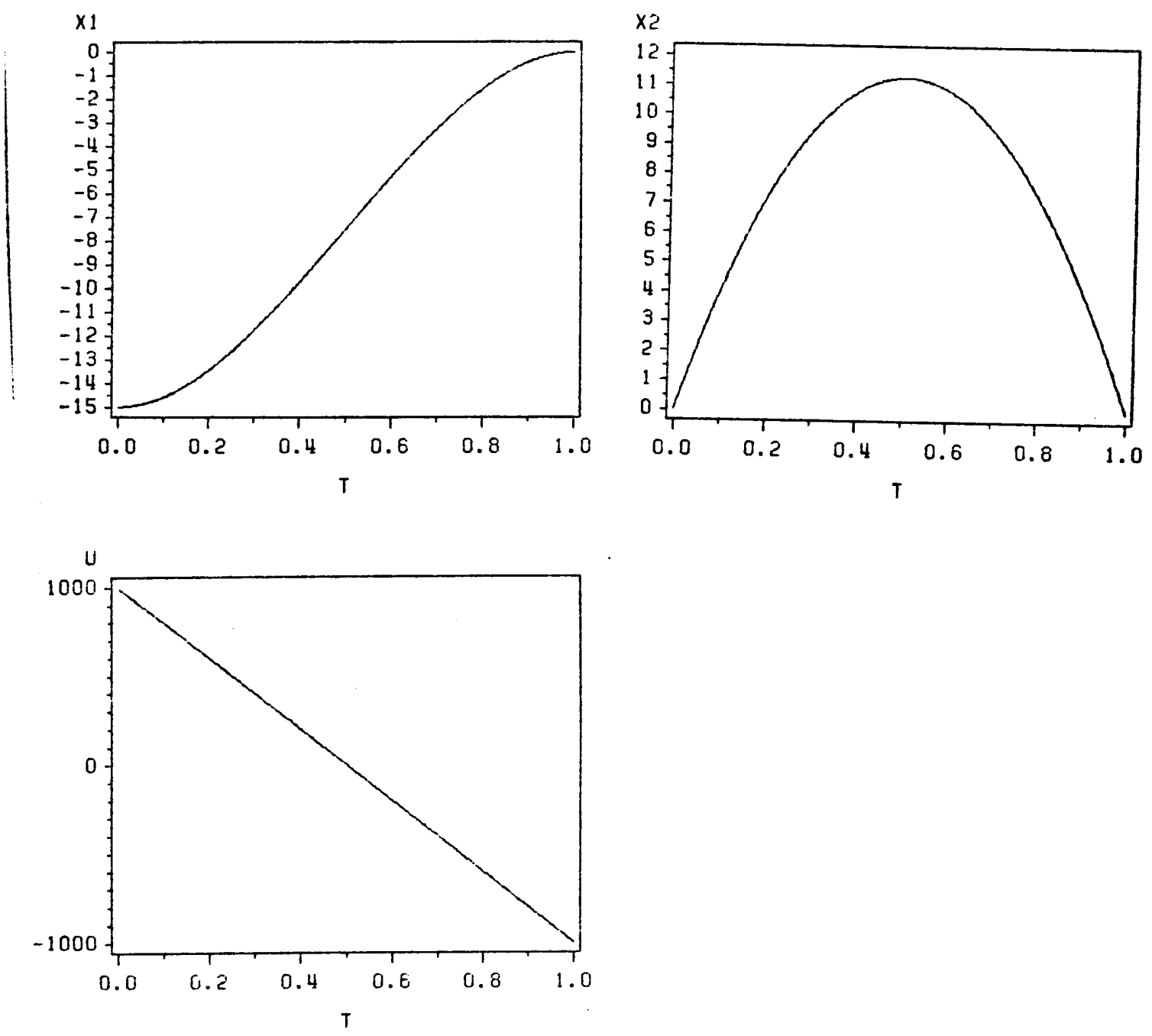

Fig. 31 : Rigid Body - Rest-to-Rest $\left(x^{\circ}=15\right)$ 

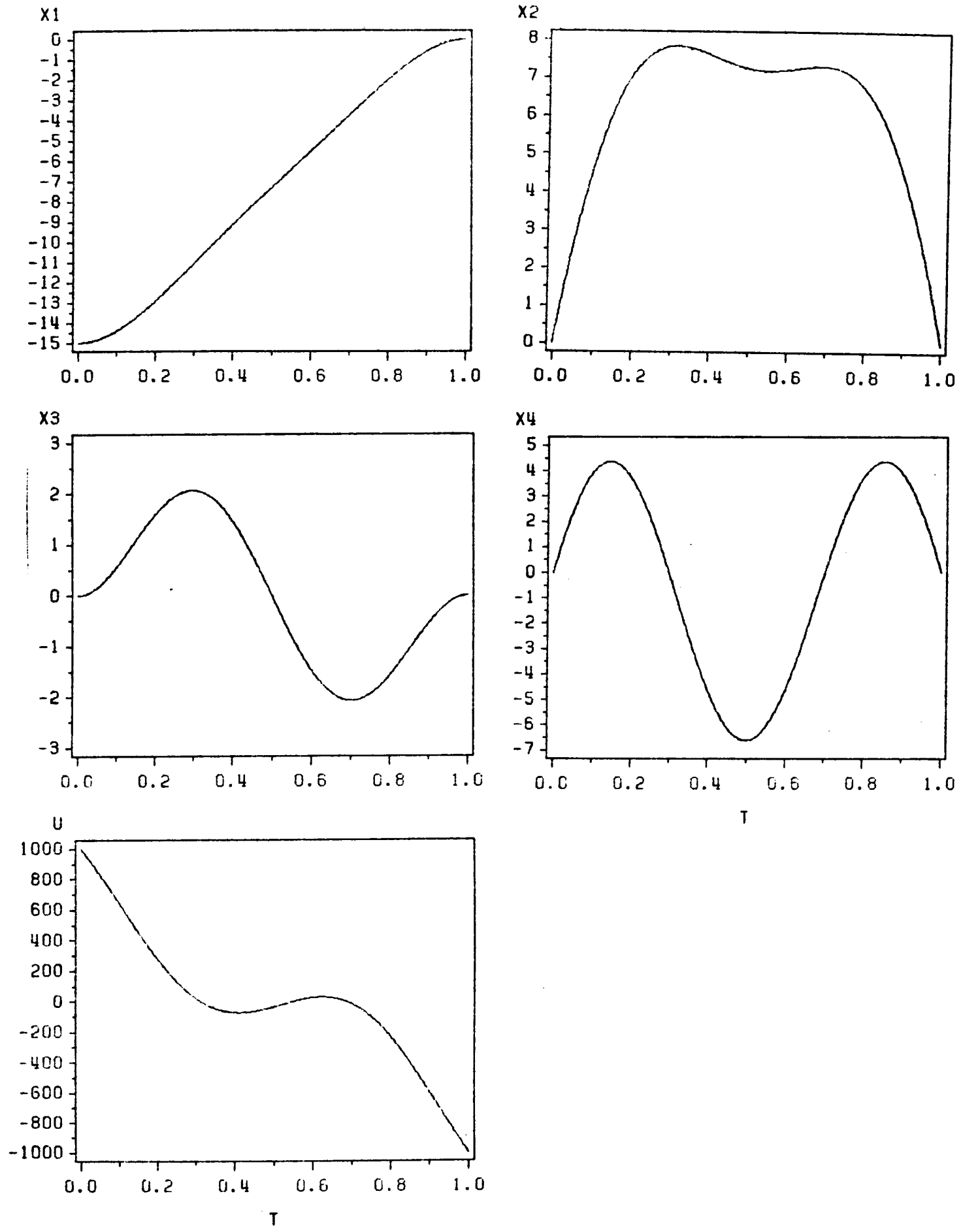

Fig. 32: One Elastic Mode - Rest-to-Rest $\left(x^{\circ}=15\right)$ 

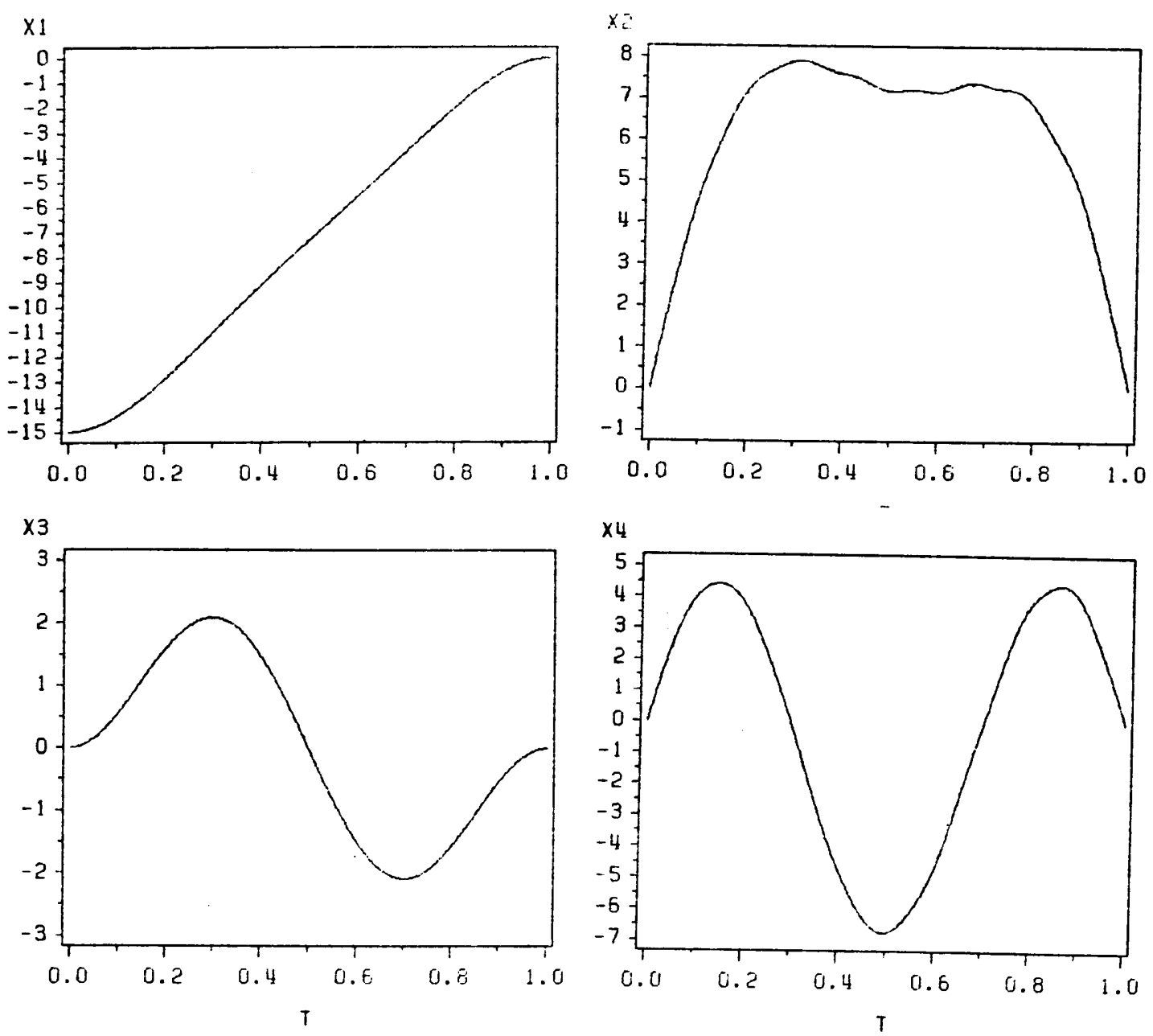

Fig. 33 : Two Elastic Modes - Rest-to-Rest $\left(x^{\circ}=15\right)$ 

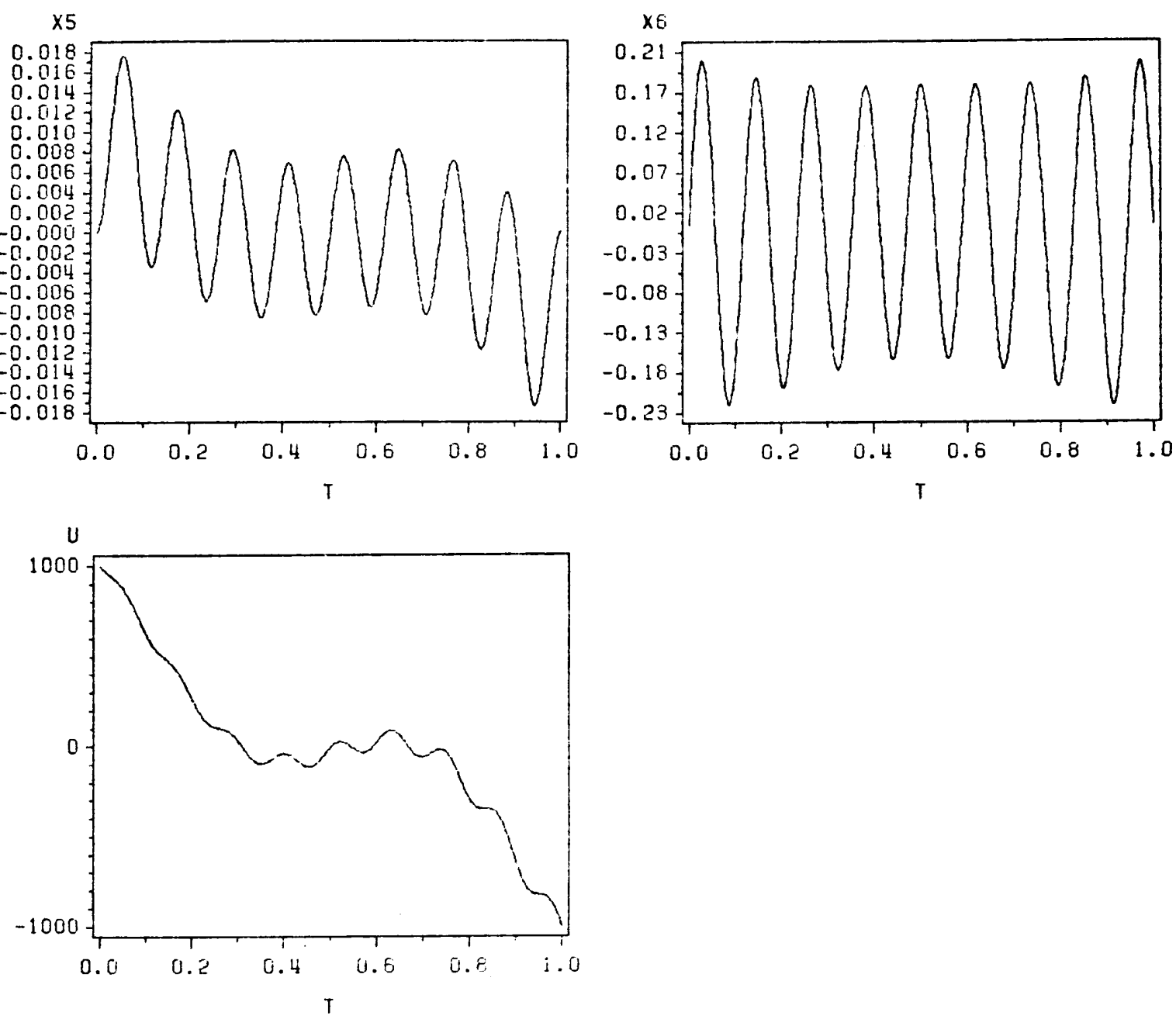

Fig. 33 : Two Elastic Modes - Rest-to-Rest $\left(x^{o}=15\right)$ (continued) 

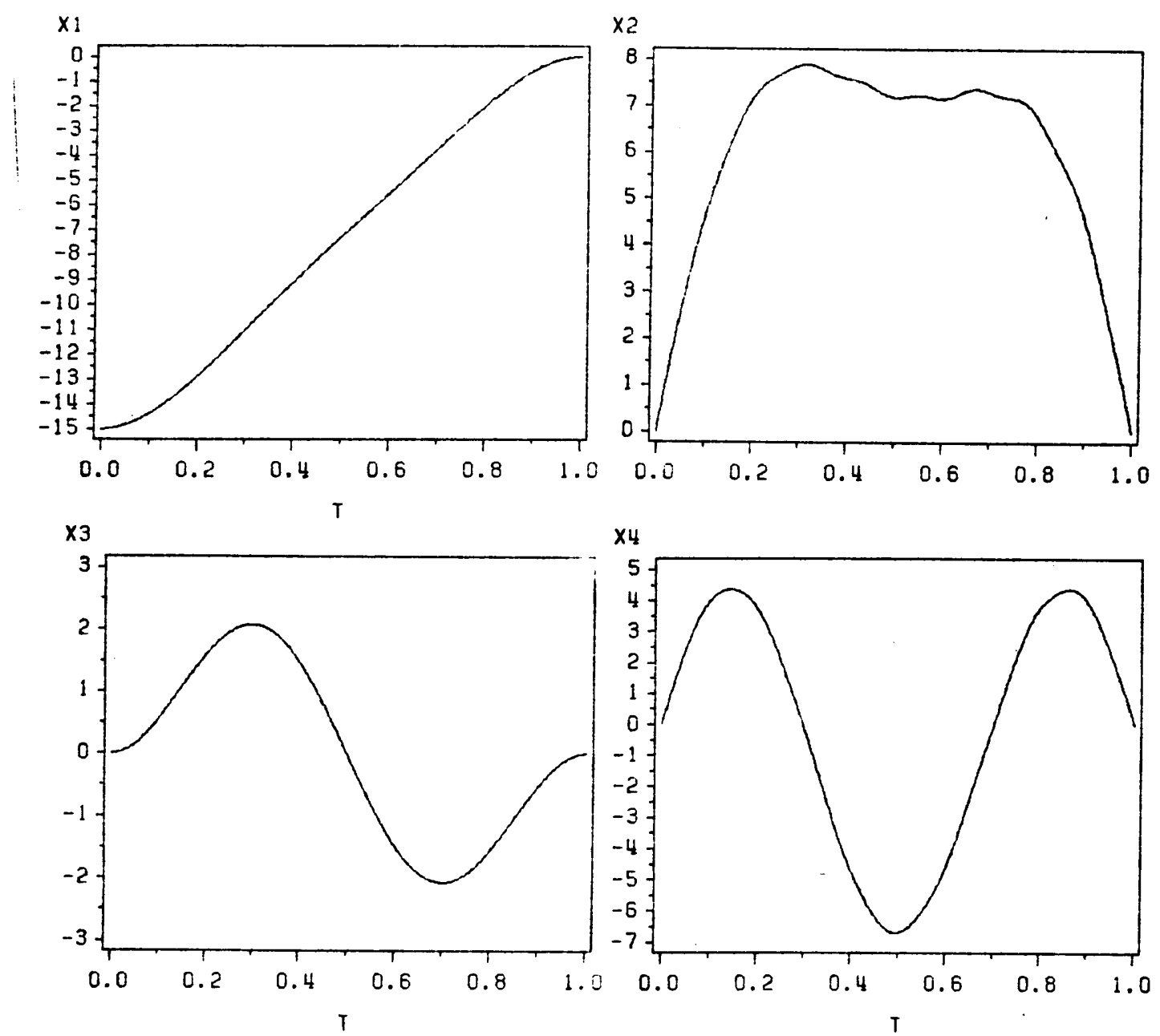

Fig. 34 : Two Elastic Modes - Rest-to-Rest (Time Fixed) 

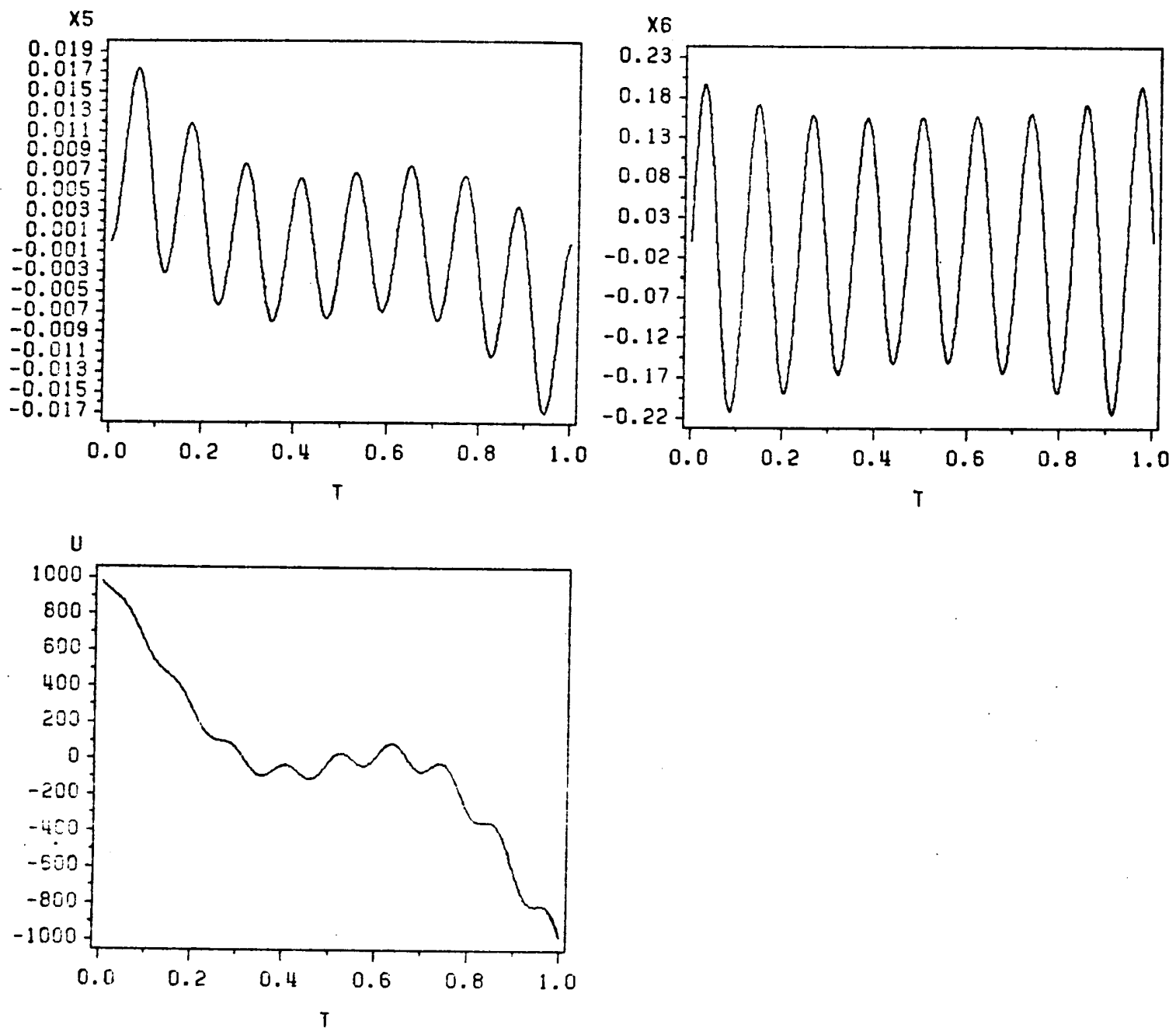

Fig. 34 : Two Elastic Modes - Rest-to-Rest (Time Fixed) (continued) 

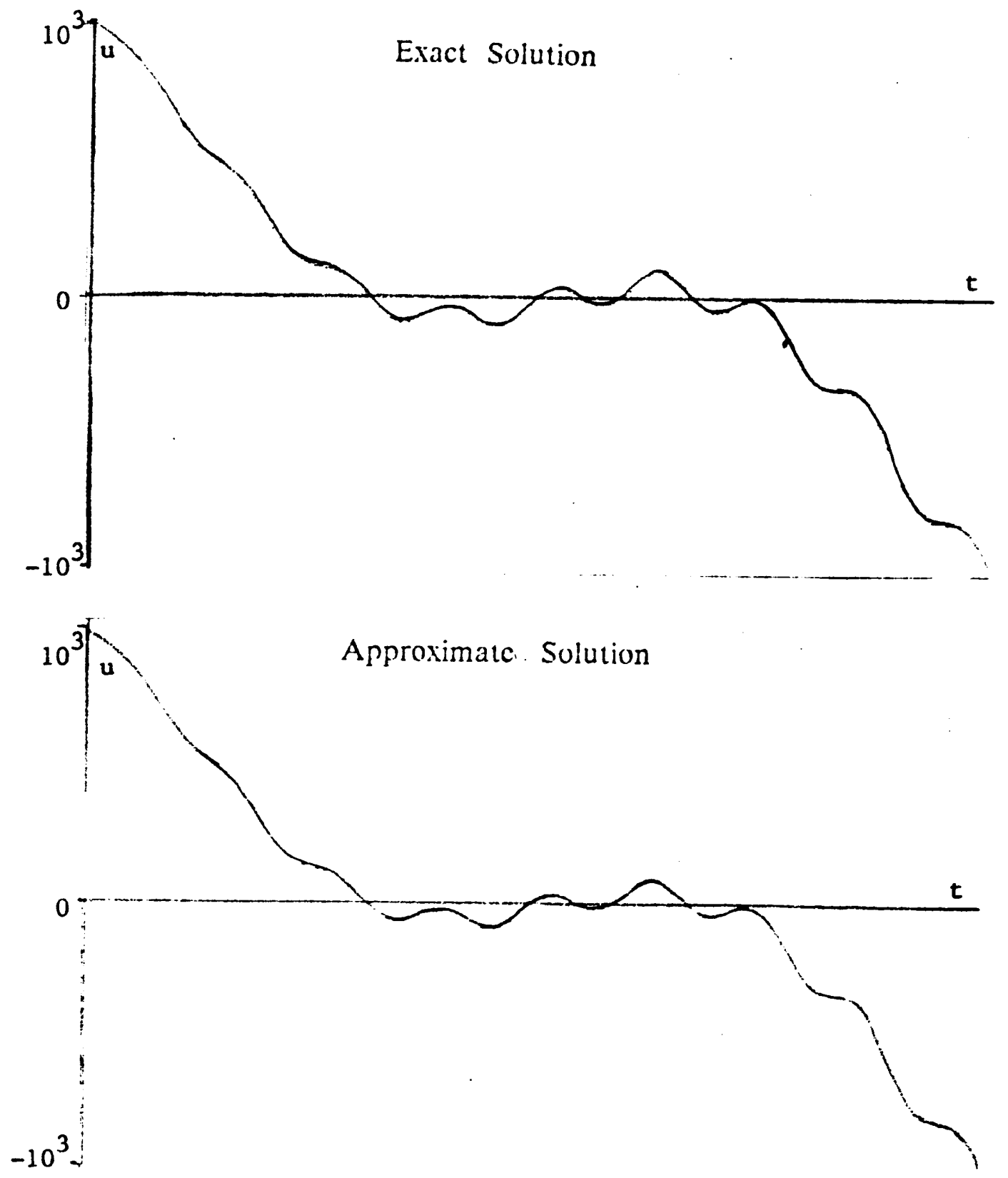

Fig. 35 : Linear System - Optimal Control Functions 

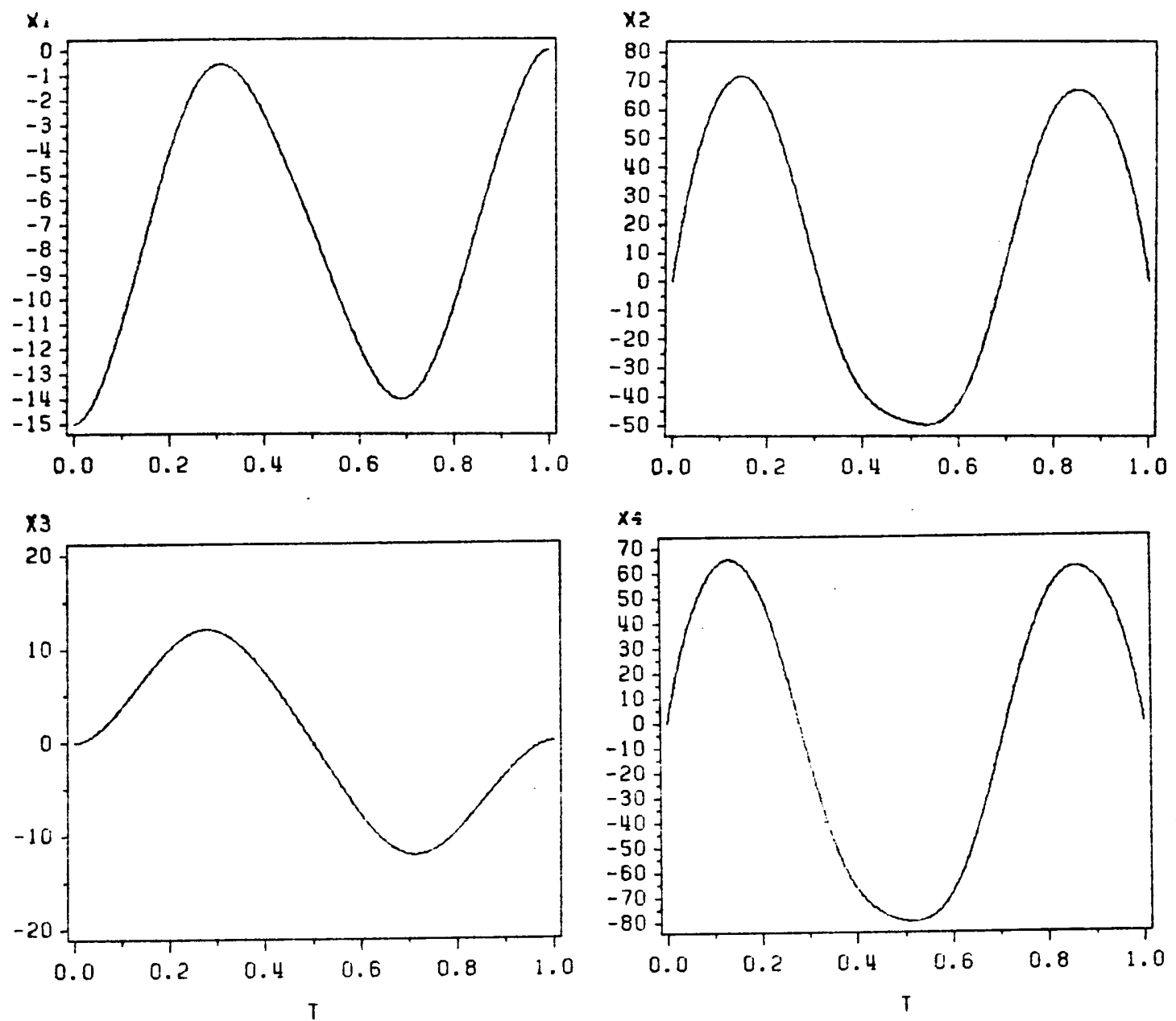

Fig. 36 : Linear Systern - Minimum Effort Problem 

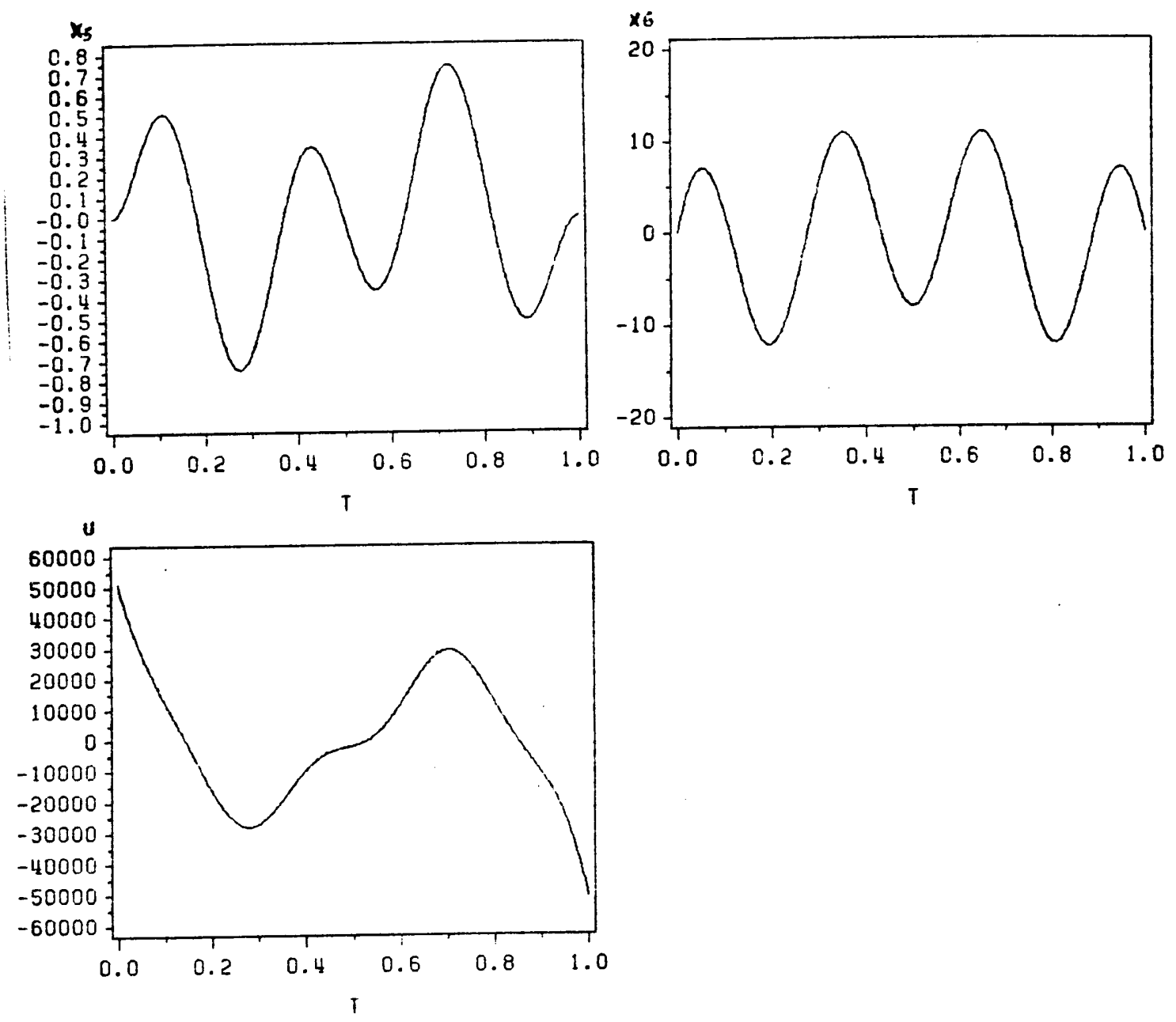

Fig. 36 : Linear System - Minimum Effort Problem (continued) 

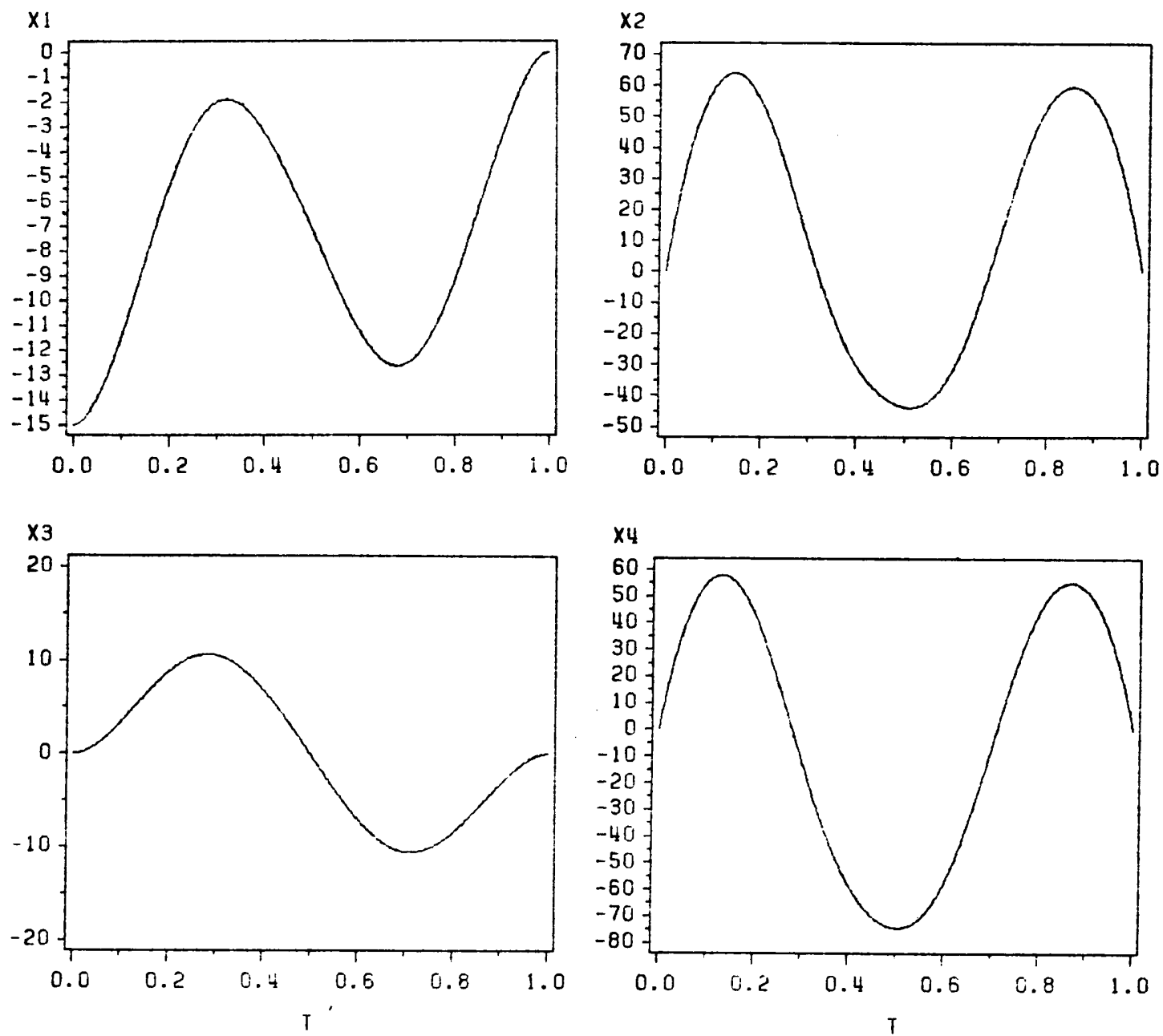

Fig. 37 : Nonlinear System - Minimum Effort Problem 

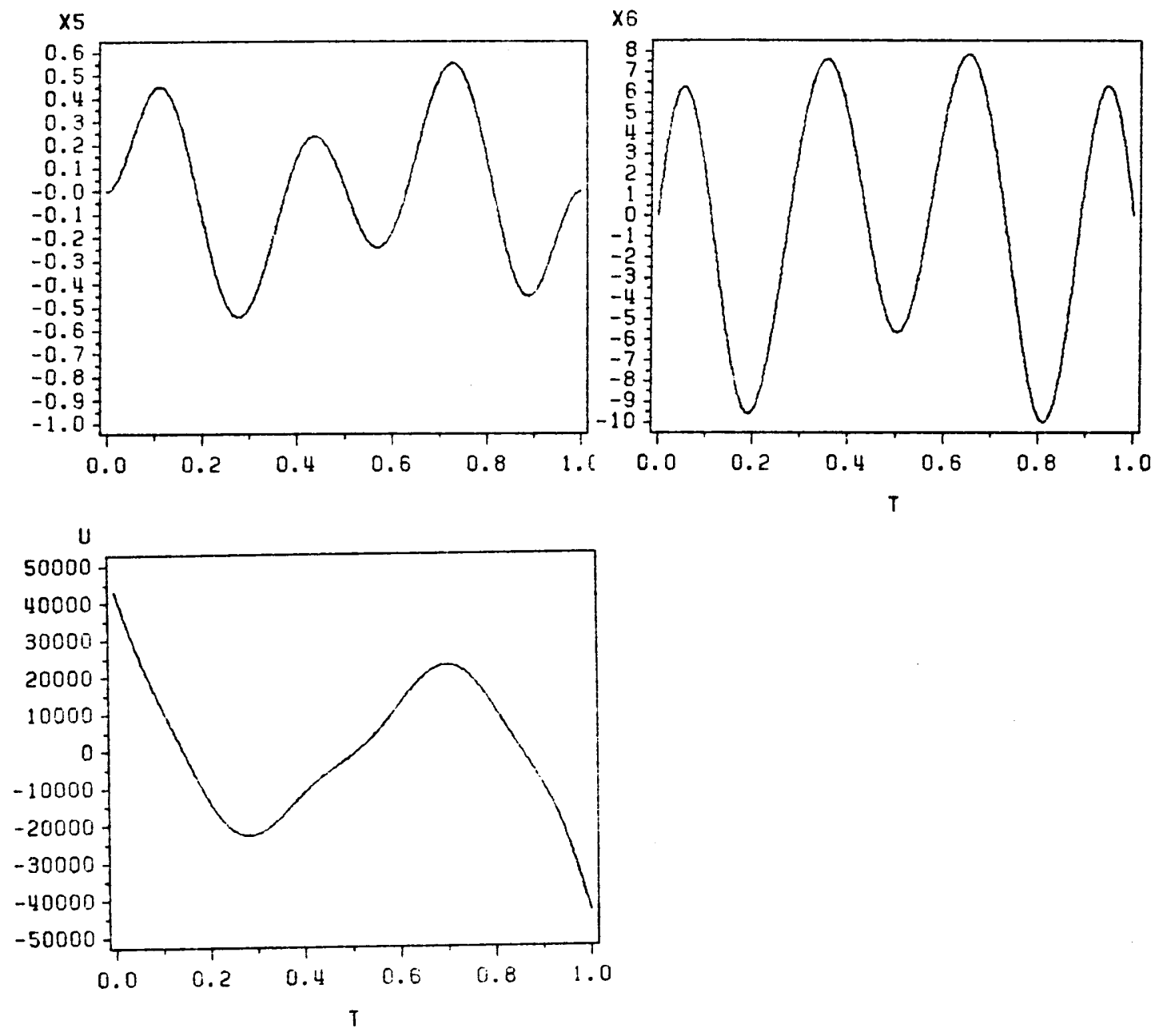

Fig. 37 : Nonlinear System - Minimum Effort Problem (continued) 

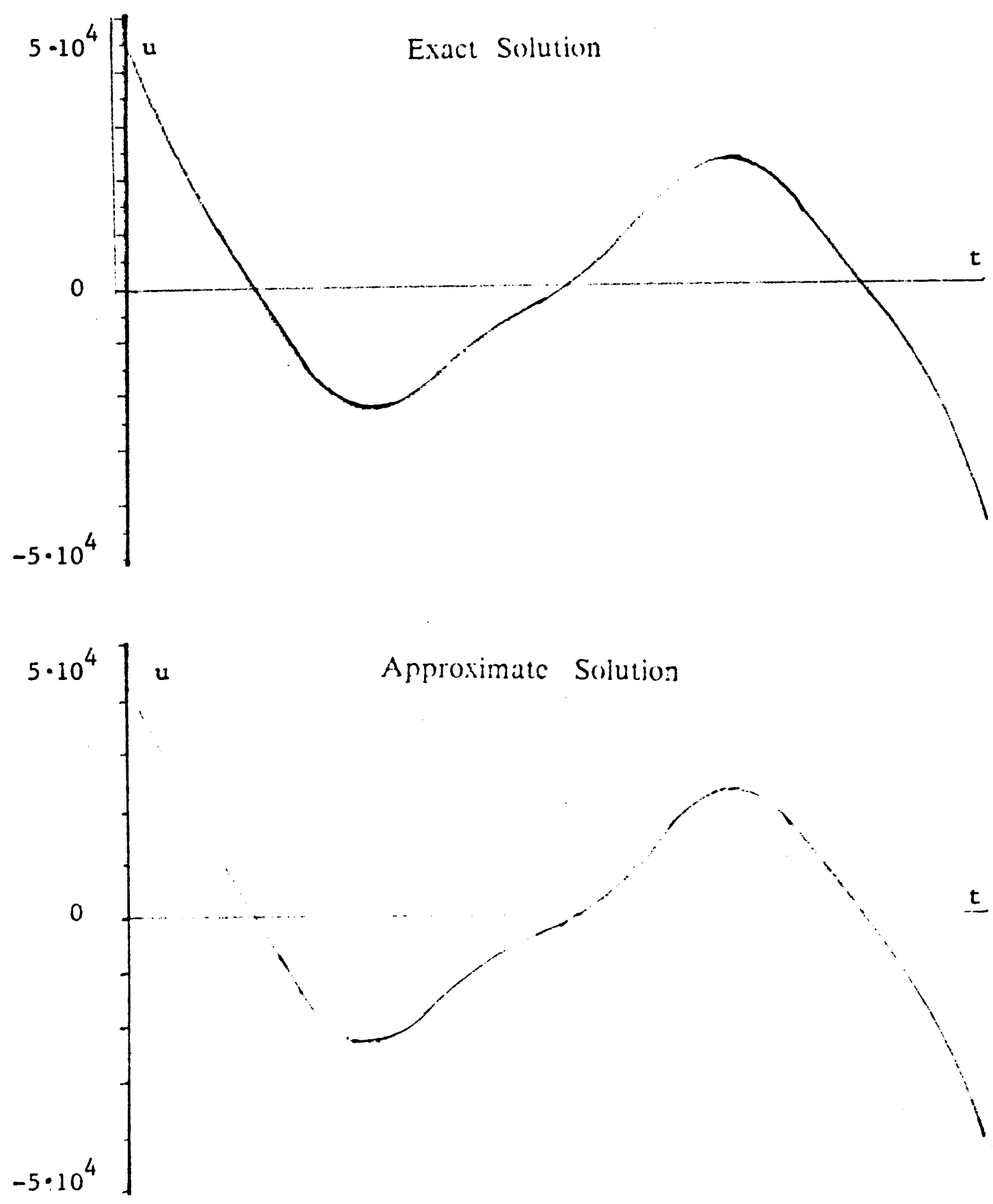

Fig. 38 : Nonlinear System - Optimal Control Functions 
The vita has been removed from the scanned document 